\title{
Galois representations with open image
}

\author{
Ralph Greenberg ${ }^{\dagger}$
}

\section{Introduction.}

Suppose that $p$ is a prime and that $n \geq 1$. Let $G_{\mathbf{Q}}=\operatorname{Gal}(\overline{\mathbf{Q}} / \mathbf{Q})$ be the absolute Galois group of Q. Our objective in this paper is to construct continuous representations

$$
\rho: G_{\mathbf{Q}} \longrightarrow G L_{n}\left(\mathbf{Z}_{p}\right)
$$

whose image is open. Continuous $n$-dimensional representations $\rho$ arise naturally in algebraic geometry for every value of $n$, but it seems difficult to find such examples where the image is open when $n \geq 3$. The construction described in this paper is not at all geometric in nature. It depends on the structure of certain Galois groups and of certain subgroups of $G L_{n}\left(\mathbf{Z}_{p}\right)$. We assume always that $p$ is an odd prime. One typical result is the following.

Proposition 1.1. Suppose that $p$ is a regular prime and that $p \geq 4\left[\frac{n}{2}\right]+1$. Let $K=\mathbf{Q}\left(\mu_{p}\right)$ and let $M$ denote the maximal pro-p extension of $K$ which is unramified outside of $p$. Then there exist continuous representations $\rho: \operatorname{Gal}(M / \mathbf{Q}) \rightarrow G L_{n}\left(\mathbf{Z}_{p}\right)$ with an open image.

A theorem of Shafarevich shows that if $p$ is a regular prime, then $\operatorname{Gal}(M / K)$ is a free pro- $p$ group on $\frac{p+1}{2}$ generators. On the other hand, it turns out that a Sylow pro- $p$ subgroup $S_{0}$ of $S L_{n}\left(\mathbf{Z}_{p}\right)$ requires only $n$ generators topologically. One can then define a surjective homomorphism $\sigma_{0}$ from $\operatorname{Gal}(M / K)$ to $S_{0}$ if $p \geq 2 n-1$. There are many choices. However, one must make the definition carefully enough so that $\sigma_{0}$ can be extended to $\operatorname{Gal}(M / \mathbf{Q})$, giving a homomorphism $\rho_{0}: \operatorname{Gal}(M / \mathbf{Q}) \rightarrow G L_{n}\left(\mathbf{Z}_{p}\right)$. If $n$ is even, one needs the slightly stronger inequality $p \geq 2 n+1$ to make that possible. The image of $\rho_{0}$ will then contain $S_{0}$ as a subgroup whose index divides $p-1$. Tensoring $\rho_{0}$ by the cyclotomic character gives a representation $\rho$ with open image. It turns out that the construction gives uncountably many such $\rho$ 's with distinct kernels.

One can prove a similar result for more pairs $(n, p)$ by making different choices for the field $K$. A crucial assumption in our construction is that $\operatorname{Gal}(M / K)$ is a free pro- $p$ group, where $M$ is defined just as above. Such a field $K$ is said to be "p-rational". We discuss this notion in some

\footnotetext{
${ }^{\dagger}$ Research supported in part by National Science Foundation grant DMS-0200785.
} 
detail in sections 3 and 4 . One interesting choice is to take $K$ to be a compositum of quadratic fields. We state a certain conjecture in section 4.2 about the $p$-rationality of such fields. Assuming that conjecture, we can then construct representations $\rho$ with open image for any $n \geq 4$ and any odd prime $p$. (See proposition 6.2.2.) If $n=3$, one can make the construction work for a very large (and undoubtedly infinite) set of primes by choosing $K=\mathbf{Q}\left(\mu_{5}\right)$. There is a very simple criterion involving Fibonacci numbers for the $p$-rationality of that field. (See section 4.4.)

Proposition 1.1 is a special case of proposition 6.1.1, the main theorem of this paper. The proof describes a construction of representations $\rho$ with open image which have the following property: the residual representation $\bar{\rho}$ is reducible. More precisely, the image of $\bar{\rho}$ consists of upper triangular matrices. The construction is group theoretic in nature and depends crucially on the results proved in section 5 concerning the pro- $p$ group $S_{0}$. We wonder whether one can prove proposition 6.1.1 by using deformation theory of Galois representations. This is not clear since the residual representation must be chosen carefully and is reducible. However, having made such a choice, it may conceivably be possible to translate our argument into the language of deformation theory.

We discuss some possible examples where $\bar{\rho}$ is irreducible in section 7 . The main result is proposition 7.1.1. Unfortunately, part of the hypothesis in that result is that some number field $K$ of large degree is $p$-rational, something which would be difficult to verify in practice. We again approach this question from a group theoretic point of view. However, there is a close relationship between our approach here and results in [Bos] concerning deformation theory for the special case where the image of $\bar{\rho}$ has order prime to $p$. One can prove proposition 7.1.1 from either point of view, but the deformation theory approach requires a somewhat weaker hypothesis. We compare the two approaches in section 7.2.

One specific type of example where $\bar{\rho}$ is irreducible is the following. It is discussed in detail in section 7.3. Suppose that $K$ is a totally complex Galois extension of $\mathbf{Q}$ such that $\Omega=\operatorname{Gal}(K / \mathbf{Q})$ is isomorphic to the symmetric group $S_{n+1}$. If $p>n+1$, then $\Omega$ has an absolutely irreducible representation $\omega$ over $\mathbf{Q}_{p}$ of degree $n$. It is a direct summand in the obvious permutation representation of $\Omega$ of degree $n+1$. One can realize $\omega$ over $\mathbf{Z}_{p}$ and $\bar{\omega}$, the reduction of $\omega$ modulo $p$, is still absolutely irreducible. Under the assumption that $K$ is $p$-rational, we will show that there exists an $n$-dimensional representation $\rho$ of $\operatorname{Gal}(M / \mathbf{Q})$ over $\mathbf{Z}_{p}$ with open image such that $\bar{\rho} \cong \bar{\omega}$. Here $M$ is defined just as in proposition 1.1. Although it would be difficult to verify the assumption that $K$ is $p$-rational when $n \geq 3$, it is reasonable to believe that it is satisfied for all but an extremely sparse, infinite set of primes. Many extensions $K$ of $\mathbf{Q}$ exist with the specified Galois group, and varying that choice certainly increases the chance that one of them will be $p$-rational for any given prime $p$.

Galois representations with open image have already been constructed by S. Hamblen for $n=3$ and $p \equiv 8(\bmod 21)$. Such examples come from his main theorems in [Ham] showing that $n$ dimensional representations $\bar{\rho}$ of $G_{\mathbf{Q}}$ over $\mathbf{F}_{p}$ can be lifted to representations $\rho$ over $\mathbf{Z}_{p}$ under certain hypotheses. Then, for certain choices of $\bar{\rho}$, Hamblen shows that there exist liftings $\rho$ with open image. The representation $\rho$ is unramified outside a finite set of primes. His specific examples 
are at the end of [Ham]. The field $K$ is the splitting field of a certain polynomial of degree 7 and $\Omega=\operatorname{Gal}(K / \mathbf{Q})$ is the simple group of order 168 . The representation $\bar{\rho}$ is absolutely irreducible, one of the two such representations of $\Omega$ of degree 3. His construction of $\rho$ 's provides examples with certain specified local properties. We discuss a similar situation from our point of view in section 7.4 .

Another interesting source of examples has been found by M. Upton [Upt]. Her examples are 3-dimensional Galois representations of $G_{F}$, where $F$ is any number field containing $\mathbf{Q}\left(\mu_{3}\right)$. They arise in a geometric way, namely from the action of $G_{F}$ on the $p$-adic Tate module $T_{p}(J)$, where $J$ is the Jacobian variety of a Picard curve $C$ defined over $F$. The genus of $C$ is 3 and $\operatorname{End}_{F}(J)$ contains $\mathbf{Z}\left[\mu_{3}\right]$. Thus, $T_{p}(J)$ can be viewed as a free module of rank 3 over the ring $R=\mathbf{Z}\left[\mu_{3}\right] \otimes_{\mathbf{Z}} \mathbf{Z}_{p}$. There is an $R$-linear action of $G_{F}$ on $T_{p}(J)$. Choosing a basis, one obtains a homomorphism $r: G_{F} \rightarrow G L_{3}(R)$. If $p \equiv 1(\bmod 3)$, then $R \cong \mathbf{Z}_{p} \times \mathbf{Z}_{p}$ and one obtains a representation $\rho: G_{F} \rightarrow G L_{3}\left(\mathbf{Z}_{p}\right)$ by projection to either factor. Upton shows that if $\operatorname{End}_{F}(J)=\mathbf{Z}\left[\mu_{3}\right]$, then $\rho$ is surjective for all but finitely many such $p$ 's. It is likely that the image of $\rho$ is open for all such primes $p$.

The topic of this paper originally arose in connection with a project concerning Iwasawa theory for elliptic curves. It was of interest to construct examples of Galois extensions of $\mathbf{Q}$ whose Galois group is isomorphic to a certain open subgroup $H_{\infty}$ of $P G L_{2}\left(\mathbf{Z}_{p}\right)$. Such extensions play a role in the illustrations in chapters 8 and 13 of [Gr1]. The proof of proposition 1.1 for the special case $n=2$ provides many such examples when $p$ is a regular prime. The representation theory for the finite quotient groups of $H_{\infty}$ is described rather precisely in proposition 7.4.4 in the above paper. Realizing $H_{\infty}$ as a Galois group over $\mathbf{Q}$ provides infinite families of irreducible Artin representations over $\mathbf{Q}$ whose degrees and modular properties are known. Many of those Artin representations are self-dual, and that makes them especially interesting to study. The main results in this paper can be viewed in a similar way. They provide constructions of a variety of examples of $p$-adic Lie extensions of $\mathbf{Q}$. The corresponding Lie algebras are $\mathfrak{s l}_{n}\left(\mathbf{Q}_{p}\right)$ or $\mathfrak{g l}_{n}\left(\mathbf{Q}_{p}\right)$. Although we have not yet examined the question carefully, we would not be surprised if $p$-adic Lie extensions of $\mathbf{Q}$ exist with any specified finite-dimensional Lie algebra over $\mathbf{Q}_{p}$.

I am grateful to Robert Pollack for asking me if the construction that I found for $n=2$ could be extended to similarly defined subgroups of $P G L_{n}\left(\mathbf{Z}_{p}\right)$, which led to proposition 1.1. I also want to thank Sourav Sen Gupta and Robert Bradshaw who carried out searches for compositums of quadratic fields which are 3-rational. Bradshaw also showed me how to use Sage for carrying out a search concerning the $p$-rationality of $\mathbf{Q}\left(\mu_{5}\right)$. Finally, I want to acknowledge support for this research from the National Science Foundation. 


\section{Pro- $p$ groups with operators.}

Assume that $\Pi$ is a pro- $p$ group. We will always assume that $\Pi$ is topologically finitely generated. This means that we can find a finite subset $\Sigma=\left\{\pi_{1}, \ldots, \pi_{t}\right\}$ of $\Pi$ such that the subgroup $\left\langle\pi_{1}, \ldots, \pi_{t}\right\rangle$ generated by $\Sigma$ is dense in $\Pi$. Let $\Phi(\Pi)$ denote the Frattini subgroup of $\Pi$, which is defined to be the intersection of all closed subgroups of $\Pi$ of index $p$. We will refer to the quotient $\widetilde{\Pi}=\Pi / \Phi(\Pi)$ as the Frattini quotient of $\Pi$. Note that $\widetilde{\Pi}$ is an abelian group of exponent $p$. We regard $\widetilde{\Pi}$ as a vector space over $\mathbf{F}_{p}$. If $\pi \in \Pi$, then its image in $\widetilde{\Pi}$ will be denoted by $\widetilde{\pi}$. It is clear that if $\left\{\pi_{1}, \ldots, \pi_{t}\right\}$ is a topological generating set for $\Pi$, then $\left\{\widetilde{\pi}_{1}, \ldots, \widetilde{\pi}_{t}\right\}$ generates $\widetilde{\Pi}$ as an $\mathbf{F}_{p^{-}}$vector space. The Burnside Basis Theorem is the converse:

BBT: If $\left\{\widetilde{\pi}_{1}, \ldots, \widetilde{\pi}_{t}\right\}$ generates $\widetilde{\Pi}$, then $\left\{\pi_{1}, \ldots, \pi_{t}\right\}$ generates $\Pi$ topologically.

In particular, if $d=\operatorname{dim}_{\mathbf{F}_{p}}(\widetilde{\Pi})$, then $\Pi$ has a topological generating set with $d$ elements, but not fewer. Note that $d=\operatorname{dim}_{\mathbf{F}_{p}}\left(H^{1}\left(\Pi, \mathbf{F}_{p}\right)\right)$, where $\Pi$ acts trivially on $\mathbf{F}_{p}$.

Suppose that $\Pi_{1}$ and $\Pi_{2}$ are pro- $p$ groups, both topologically finitely generated. Suppose that $\sigma: \Pi_{1} \rightarrow \Pi_{2}$ is a continuous group homomorphism. Then $\sigma$ induces a homomorphism from $\widetilde{\Pi}_{1}$ to $\widetilde{\Pi}_{2}$ which we denote by $\widetilde{\sigma}$. It follows easily from BBT that $\sigma$ is surjective if and only if $\widetilde{\sigma}$ is surjective.

We will need the profinite version of the Schur-Zassenhaus theorem in this paper. It will be used several times in the following form. The group $G$ will sometimes be a Galois group, sometimes a subgroup of $G L_{n}\left(\mathbf{Z}_{p}\right)$, and sometimes a subgroup of $\operatorname{Aut}(\Pi)$, the group of continuous automorphisms of a pro- $p$ group $\Pi$.

SZT: Suppose that $G$ is a profinite group, that $N$ is a normal pro-p subgroup of $G$, and that $G / N$ is a finite group of order prime to $p$. Then $G$ contains a subgroup $H$ such that $G=H N$ and $H \cap N=\left\{i d_{G}\right\}$. Furthermore, all such subgroups of $G$ are conjugate.

The usual form of the Schur-Zassenhaus theorem concerns finite groups and can be found in [Gor], theorem 2.1. Extending it from finite to profinite groups is not difficult. Note that if $G, N$, and $H$ are as in $\mathbf{S Z T}$, then we obviously have $H \cong G / N$. The theorem means that $G$ is isomorphic to a semidirect product $N \rtimes H$, where $H$ acts on $N$ by conjugation. Furthermore, it follows that if $H$ and $H^{\prime}$ are two such subgroups, then we have $H^{\prime}=n H n^{-1}$ for some $n \in N$.

2.1. The $\Omega$-type. $\quad$ Now suppose that $\Omega$ is a finite group of order prime to $p$. Let Aut $(\Pi)$ denote the group of continuous automorphisms of $\Pi$. Suppose that we are given a homomorphism $\Omega \longrightarrow \operatorname{Aut}(\Pi)$. We will then refer to $\Pi$ as an $\Omega$-group. We can view $\widetilde{\Pi}$ as a finite-dimensional $\mathbf{F}_{p}$-representation space for $\Omega$. It must be completely reducible because $p \nmid|\Omega|$. We will refer to the isomorphism class of $\widetilde{\Pi}$ as the " $\Omega$-type" of $\Pi$. Let $\operatorname{Irr}_{\mathbf{F}_{p}}(\Omega)$ be the set of isomorphism classes of $\mathbf{F}_{p}$-irreducible representations of $\Omega$. For each $\chi$ in $\operatorname{Irr}_{\mathbf{F}_{p}}(\Omega)$, let $m_{\chi}(\widetilde{\Pi})$ denote the multiplicity of $\chi$ as a constituent in $\widetilde{\Pi}$. The $\Omega$-type of $\Pi$ is determined if one knows those multiplicities for 
all $\chi \in \operatorname{Irr}_{\mathbf{F}_{p}}(\Omega)$. Note that if $\Pi$ is an $\Omega$-group, then so is its maximal abelian quotient $\Pi^{a b}$. The $\Omega$-types of $\Pi$ and of $\Pi^{a b}$ are obviously the same.

An important hypothesis in most of our results will be that $\Omega$ satisfies the following property:

Assumption A: $\Omega$ is an abelian group and every element of $\Omega$ has order dividing $p-1$.

If this assumption is satisfied, then $\operatorname{Irr}_{\mathbf{F}_{p}}(\Omega)$ can be identified with $\widehat{\Omega}=\operatorname{Hom}\left(\Omega, \mathbf{F}_{p}^{\times}\right)$. There is a canonical homomorphism $\mathbf{F}_{p}^{\times} \rightarrow \mathbf{Z}_{p}^{\times}$since every coset in $\left(\mathbf{Z}_{p} / p \mathbf{Z}_{p}\right)^{\times}$contains a unique $(p-1)$-st root of unity. If $\chi \in \widehat{\Omega}$, then composing with that canonical homomorphism gives a character of $\Omega$ with values in $\mathbf{Z}_{p}^{\times}$. We will simply use the same letter $\chi$ for that lifting. If $\pi \in \Pi$ and $a \in \mathbf{Z}_{p}$, then one can define $\pi^{a}$. It is an element in the closure of the subgroup $\langle\pi\rangle$ generated by $\pi$, which we denote by $\overline{\langle\pi\rangle}$. Thus, it makes sense to write $\pi^{\chi(\alpha)}$ if $\pi \in \Pi, \alpha \in \Omega$, and $\chi \in \widehat{\Omega}$.

Assume that $\Pi$ is a pro- $p \Omega$-group and that $\Omega$ satisfies assumption $\mathbf{A}$. We will describe a useful refinement of the Burnside Basis Theorem in this case. For $\alpha \in \Omega$ and $\pi \in \Pi$, we write $\alpha(\pi)$ for the image of $\pi$ under the automorphism of $\Pi$ corresponding to $\alpha$. Let $\chi \in \widehat{\Omega}$. A nontrivial element $\pi \in \Pi$ will be called a " $\chi$-element" if $\alpha(\pi)=\pi^{\chi(\alpha)}$ for all $\alpha \in \Omega$. We will also refer to such an element $\pi \in \Pi$ as an $\Omega$-element if we don't specify the character $\chi$. This simply means that $\overline{\langle\pi\rangle}$ is invariant under the action of $\Omega$. One can find an $\mathbf{F}_{p}$-basis for $\widetilde{\Pi}$ consisting of $\Omega$-elements. The following result together with BBT implies that one can lift such a basis to a set of topological generators for $\Pi$ consisting of $\Omega$-elements.

Proposition 2.1.1. Suppose that $\Omega$ satisfies assumption A. Suppose that $\chi$ is a character of $\Omega$ and that $z$ is a $\chi$-element in $\widetilde{\Pi}$. Then there exists a $\chi$-element $x$ in $\Pi$ such that $\widetilde{x}=z$.

This result can be found in [Bos]. It is a special case of proposition 2.3 in that paper, as is noted on page 184. We also had discovered it prior to learning that it was already in [Bos] since we needed it for proving proposition 1.1. It plays a central role in this paper and so we will give our proof. It is somewhat different than the argument found in [Bos], although essentially as simple.

Proof. First of all, assume that $\Pi$ is abelian. Then $\Phi(\Pi)=\Pi^{p}$. We can regard $\Pi$ and $\widetilde{\Pi}=$ $\Pi / \Pi^{p}$ as $\mathbf{Z}_{p}[\Omega]$-modules. We will use an exponential notation for the action of $\mathbf{Z}[\Omega]$ on $\Pi$, writing $x^{\theta}$ for $x \in \Pi$ and $\theta \in \mathbf{Z}_{p}[\Omega]$. We use the same notation for $\widetilde{\Pi}$. Let $e_{\chi} \in \mathbf{Z}_{p}[\Omega]$ denote the idempotent for $\chi$. Then $\Pi^{e_{\chi}}$ is a direct summand of $\Pi$ as a $\mathbf{Z}_{p}[\Omega]$-module. We refer to $\Pi^{e_{\chi}}$ as the $\chi$-component of $\Pi$. The $\chi$-elements of $\Pi$ are the non-trivial elements in $\Pi^{e_{\chi}}$. The natural map $\Pi \rightarrow \widetilde{\Pi}$ induces a homomorphism $\Pi^{e_{\chi}} \longrightarrow \widetilde{\Pi}^{e_{\chi}}$ which is clearly surjective. Thus, the stated result is true if $\Pi$ is abelian. Furthermore, if $\Psi$ is a subgroup of $\Pi^{p}$ and $\Pi^{\prime}=\Pi / \Psi$, then we have surjective homomorphisms $\Pi \rightarrow \Pi^{\prime} \rightarrow \widetilde{\Pi}$. The corresponding homomorphisms on the $\chi$-components are also surjective. Thus, for any $\chi$-element $x^{\prime}$ of $\Pi^{\prime}$ which maps to $z$, there exists a $\chi$-element $x$ of $\Pi$ which maps to $x^{\prime}$, and hence to $z$.

Now assume that $\Pi$ is a finite $p$-group, but not necessarily abelian. We prove by induction on $|\Pi|$ that there exists a $\chi$-element $x \in \Pi$ such that $\widetilde{x}=z$. We may suppose that $|\Phi(\Pi)|>1$. Let 
$\mathcal{Z}$ denote the center of $\Pi$ and let $\Psi=\mathcal{Z} \cap \Phi(\Pi)$. Then $\Psi$ is a normal, $\Omega$-invariant subgroup of $\Pi$, $\Psi \subseteq \Phi(\Pi)$, and $|\Psi|>1$. Let $\Pi^{\prime}=\Pi / \Psi$. Then we can identify $\widetilde{\Pi^{\prime}}$ with $\widetilde{\Pi}$. Assume (inductively) that we can find a $\chi$-element $x^{\prime}$ in $\Pi^{\prime}$ whose image in $\widetilde{\Pi}$ is $z$. Then $x^{\prime}=y \Psi$, where $y \in \Pi$ and $\alpha(y) \equiv y^{\chi(\alpha)}(\bmod \Psi)$ for all $\alpha \in \Omega$. The image of $y$ in $\widetilde{\Pi}$ is $z$. The subgroup $\langle\Psi, y\rangle$ generated by $\Psi$ and $y$ will be $\Omega$-invariant and abelian. As already shown, one can find a $\chi$-element $x \in\langle\Psi, y\rangle$ such that $x \equiv y(\bmod \Psi)$, establishing the lemma if $\Pi$ is finite.

We use a similar argument if $\Pi$ is infinite. We can find a descending sequence of open, normal, $\Omega$-invariant subgroups $\Psi_{j}$ of $\Pi$, all contained in $\Phi(\Pi)$, such that $\bigcap_{j} \Psi_{j}=\left\{i d_{\Pi}\right\}$ and $\Psi_{j} / \Psi_{j+1}$ is contained in the center of $\Pi / \Psi_{j+1}$. We obtain $x \in \Pi$ with the desired properties as a compatible sequence of suitable elements $x_{j} \in \Pi / \Psi_{j}$.

Apart from Boston's paper mentioned above, the existence of a generating set consisting of $\Omega$-elements has been pointed out elsewhere in the special case where $\Omega$ has order 2 . For example, Herfort and Ribes [HeRi] show that if $\Pi$ is a pro- $p$-group with an involution, and $p$ is an odd prime, then $\Pi$ has a set of topological generators which are either fixed or inverted by the given involution.

Assumption A will prevail throughout most of this paper. However, we sometimes will want to make the following weaker assumption, especially in section 7 .

Assumption B. The order of $\Omega$ is not divisible by $p$.

Roughly speaking, the role of this assumption is partly that it makes the relationship between representation theory for $\Omega$ in characteristic 0 and in characteristic $p$ quite simple. Also, it allows us to apply the SZT in several situations, e.g., the proof of proposition 2.3.1 below.

2.2. Free pro- $p \Omega$-groups. Returning to the case where $\Omega$ is any finite group of order prime to $p$, suppose that $\Gamma$ is a free pro- $p$ group on $d$ generators and that one is given a homomorphism $\psi: \Omega \rightarrow \operatorname{Aut}_{\mathbf{F}_{p}}(\widetilde{\Gamma})$. Of course, that homomorphism is just a $d$-dimensional representation of $\Omega$ over $\mathbf{F}_{p}$. We want to now show that $\psi$ can be lifted to a homomorphism $\varphi: \Omega \rightarrow \operatorname{Aut}(\Gamma)$. As a consequence, one can find a free $\Omega$-group $\Gamma$ with any specified $\Omega$-type.

First of all, observe that any automorphism $\widetilde{\alpha}$ of $\widetilde{\Gamma}$ can be lifted to a continuous automorphism $\alpha$ of $\Gamma$. That is, the natural map

$$
\operatorname{Aut}(\Gamma) \longrightarrow \operatorname{Aut}(\widetilde{\Gamma})
$$

is surjective. To see this, suppose that $\Gamma$ is the free pro- $p$ group on the set $\Sigma=\left\{\gamma_{1}, \ldots, \gamma_{t}\right\}$. The universal mapping property for $(\Gamma, \Sigma)$ implies that we can at least define a continuous homomorphism $\alpha: \Gamma \rightarrow \Gamma$ lifting $\widetilde{\alpha}$. The surjectivity of $\alpha$ follows from BBT. The inflation-restriction sequence together with the fact that $H^{2}\left(\Gamma, \mathbf{F}_{p}\right)=0$ implies easily that $H^{1}\left(\operatorname{ker}(\alpha), \mathbf{F}_{p}\right)=0$. Since $\operatorname{ker}(\alpha)$ is a pro- $p$ group, it follows that $\operatorname{ker}(\alpha)$ is trivial and hence that $\alpha$ is injective. The continuity of $\alpha^{-1}$ also follows easily. 
Let $N$ denote the kernel of the map (1). It is known that $N$ is a pro- $p$ group. In fact, this is true if $\Gamma$ is any topologically finitely-generated pro- $p$ group. (See proposition 5.5 in [DSMS].) Let $G \subset \operatorname{Aut}(\Gamma)$ be the inverse image of $\psi(\Omega)$ under the map (1). Thus, $G$ contains $N$ and the corresponding quotient group $G / N$ is isomorphic to $\psi(\Omega)$. Since $\psi(\Omega)$ has order prime to $p$, SZT tells us that $G$ is a semi-direct product. That is, $G$ contains a subgroup $H$ such that $G=H N$ and $H \cap N=\left\{i d_{G}\right\}$. Thus, the obvious map $H \rightarrow G / N$ is an isomorphism and therefore we have a uniquely determined surjective map $\varphi: \Omega \rightarrow H$ which induces the map $\psi: \Omega \rightarrow G / N \rightarrow \operatorname{Aut}(\widetilde{\Gamma})$, as we wanted. Since $H \subset \operatorname{Aut}(\Gamma)$, the map $\varphi$ makes $\Gamma$ into an $\Omega$-group.

Remark 2.2.1. A theorem in modular representation theory asserts that if $\Omega$ has order prime to $p$, then any homomorphism $\bar{\omega}: \Omega \rightarrow G L_{d}\left(\mathbf{F}_{p}\right)$ can always be lifted to a homomorphism $\omega: \Omega \rightarrow$ $G L_{d}\left(\mathbf{Z}_{p}\right)$. In fact, the same argument as above shows this. The map $G L_{d}\left(\mathbf{Z}_{p}\right) \rightarrow G L_{d}\left(\mathbf{F}_{p}\right)$ is easily seen to be surjective. That is all one needs in the above argument. Alternatively, in the above notation, one can choose an $\mathbf{F}_{p}$-basis for $\widetilde{\Gamma}$ and identify $\bar{\omega}$ with $\psi$. One can lift the chosen basis for $\widetilde{\Gamma}$ to a set of topological generators $\Sigma$ for $\Gamma$. The homomorphism $\varphi$ induces a homomorphism

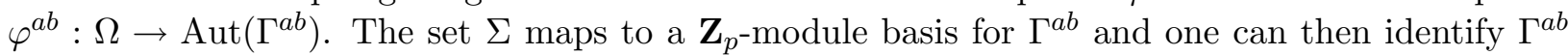
with $\mathbf{Z}_{p}^{d}$. Then $\varphi^{a b}$ defines a homomorphism $\omega$ which lifts $\bar{\omega}$.

If $\Omega$ has order prime to $p$ and $L$ is a free $\mathbf{Z}_{p}$-module of finite rank on which $\Omega$ acts, then $L$ is a $\mathbf{Z}_{p}[\Omega]$-module. It is a projective module and its isomorphism class determines and is determined by the isomorphism class of $L / p L$ as an $\mathbf{F}_{p}[\Omega]$-module. Furthermore, the isomorphism class of the $\mathbf{Q}_{p}[\Omega]$-module $L \otimes \mathbf{z}_{p} \mathbf{Q}_{p}$ determines and is determined by the isomorphism class of $L$ as a $\mathbf{Z}_{p}[\Omega]$ module. One can find these useful results in [Ser], specifically in proposition 43 and in corollary 2 to theorem 34 .

One consequence that we will need is the following. Suppose that $\Gamma$ is a free pro- $p \Omega$-group.

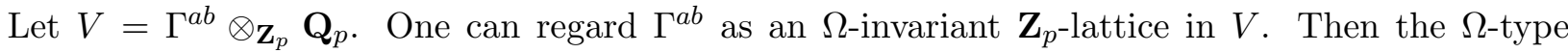
of $\Gamma$ determines and is determined by the isomorphism class of the $\mathbf{Q}_{p}$-representation space $V=$ $\Gamma^{a b} \otimes \mathbf{z}_{p} \mathbf{Q}_{p}$ for $\Omega$.

The simplest examples of free pro- $p \Omega$-groups occur when $d=1$ and $\Omega$ is an abelian group of exponent dividing $p-1$. Let $\chi \in \widehat{\Omega}$. Regarding $\chi$ as having values in $\mathbf{Z}_{p}^{\times}$, we let $\Gamma_{\chi}$ denote a group isomorphic to $\mathbf{Z}_{p}$ on which $\Omega$ acts by $\chi$. We can obviously take direct products of such groups, obtaining $\Omega$-groups which are free, abelian pro- $p$ groups and which have any specified $\Omega$-type. One can also take a free product of finitely many such groups, which gives an explicit way of obtaining a free pro- $p \Omega$-group with any specified $\Omega$-type in the special case where $\Omega$ satisfies assumption $\mathbf{A}$.

2.3. A universal mapping property. We only assume that $\Omega$ satisfies assumption B. Suppose that $\Pi$ is a finitely-generated pro- $p$ group which is also an $\Omega$-group. The following result gives the existence of surjective $\Omega$-homomorphisms from a free pro- $p \Omega$-group $\Gamma$ to $\Pi$ if the $\Omega$-type of $\Pi$ is "bounded above" by the $\Omega$-type of $\Gamma$. 
Proposition 2.3.1. Suppose that $\Gamma$ is a free pro-p $\Omega$-group on a finite number of generators, that $\Pi$ is a pro-p $\Omega$-group, and that there exists a surjective $\Omega$-homomorphism

$$
\tau: \widetilde{\Gamma} \rightarrow \widetilde{\Pi}
$$

Then there exists a surjective $\Omega$-homomorphism $\sigma: \Gamma \rightarrow \Pi$ such that $\widetilde{\sigma}=\tau$.

Note that such a $\tau$ exists if and only if $m_{\chi}(\widetilde{\Pi}) \leq m_{\chi}(\widetilde{\Gamma})$ for all $\chi \in \operatorname{Irr}_{\mathbf{F}_{p}}(\Omega)$.

Proof. The structure of $\Gamma$ as an $\Omega$-group is given by a homomorphism $\varphi: \Omega \rightarrow \operatorname{Aut}(\Gamma)$. Let $H$ denote the image of $\varphi$. Let $\psi: \Omega \rightarrow \operatorname{Aut}(\widetilde{\Gamma})$ be the homomorphism induced by $\varphi$. Note that $H$ is mapped injectively into Aut $(\widetilde{\Gamma})$ and that the image of $H$ under that map coincides with $\psi(\Omega)$. The structure of $\Pi$ as an $\Omega$-group is given by a homomorphism $\kappa: \Omega \rightarrow \operatorname{Aut}(\Pi)$. Let $J$ denote the image of $\kappa$. Let $\lambda: \Omega \rightarrow \operatorname{Aut}(\widetilde{\Pi})$ be the homomorphism induced by $\kappa$. With this notation, we have $\tau \circ \psi(\alpha)=\lambda(\alpha) \circ \tau$ for all $\alpha \in \Omega$.

We will assume at first that $\tau$ is an isomorphism. Thus, $\tau$ induces an isomorphism from Aut $(\widetilde{\Gamma})$ to $\operatorname{Aut}(\widetilde{\Pi})$ which sends $\psi(\Omega)$ to $\lambda(\Omega)$. Since $J$ is mapped injectively into Aut $(\widetilde{\Pi})$, it is clear that $\tau$ induces an isomorphism $H \rightarrow J$. Also, BBT implies that there is a continuous, surjective homomorphism $\delta: \Gamma \rightarrow \Pi$ such that $\widetilde{\delta}=\tau$. Let $\Delta=\operatorname{ker}(\delta)$. Let $\operatorname{Aut}(\Gamma, \Delta)$ denote the group of continuous automorphisms of $\Gamma$ fixing the subgroup $\Delta$. Thus, $\operatorname{Aut}(\Gamma, \Delta)$ is a subgroup of $\operatorname{Aut}(\Gamma)$ and we have a homomorphism

$$
\operatorname{Aut}(\Gamma, \Delta) \longrightarrow \operatorname{Aut}(\Pi)
$$

whose kernel $N^{\prime}$ is a subgroup of the kernel of (1) and hence is a pro- $p$ subgroup of $\operatorname{Aut}(\Gamma, \Delta)$. One also sees easily that (2) is surjective, just as for the map (1). Let $G^{\prime}$ denote the inverse image of $J$ under (2). Thus, $G^{\prime} / N^{\prime} \cong J$. It follows from SZT that $G^{\prime}$ contains a subgroup $H^{\prime}$ such that $G^{\prime}=H^{\prime} N^{\prime}$ and $H^{\prime} \cap N^{\prime}=\left\{i d_{G^{\prime}}\right\}$. It is clear that (2) maps $H^{\prime}$ isomorphically to $J$. Moreover, the map $\kappa: \Omega \rightarrow J$ determines a map $\varphi^{\prime}: \Omega \rightarrow H^{\prime}$. If we regard $\Gamma$ as an $\Omega$-group by using the map $\varphi^{\prime}$ (instead of using $\varphi$ ), then $\delta$ becomes a surjective $\Omega$-homomorphism from $\Gamma$ to $\Pi$.

Note that $\varphi$ and $\varphi^{\prime}$ induce the same map from $\Omega$ to $\operatorname{Aut}(\widetilde{\Gamma})$, namely the map $\psi$. As previously, let $N$ be the kernel of (1) and let $G$ be the inverse image of $\psi(\Omega)$. Then $H^{\prime} \subset G$ and $G=H^{\prime} N$. It follows from SZT that $H$ and $H^{\prime}$ are conjugate subgroups of $G$. More precisely, there is an element $\eta \in N$ such that $H^{\prime}=\eta H \eta^{-1}$. Consequently, if $\alpha \in \Omega$, then $\varphi^{\prime}(\alpha) \circ \eta=\eta \circ \varphi(\alpha)$. Also, $\eta$ induces the identity map on $\widetilde{\Gamma}$.

The above remarks show that $\sigma=\delta \circ \eta$ is a surjective $\Omega$-homomorphism from $\Gamma$ to $\Pi$ and that $\widetilde{\sigma}$ coincides with the isomorphism $\tau$ from $\widetilde{\Gamma}$ to $\widetilde{\Pi}$. To complete the proof, suppose now that $\tau$ is not injective. Then one can define an isomorphism

$$
\tau_{1}: \widetilde{\Gamma} \longrightarrow \widetilde{\Pi} \times \operatorname{ker}(\tau)
$$


of representation spaces for $\Omega$ such that composing $\tau_{1}$ with projection to the factor $\widetilde{\Pi}$ gives the map $\tau$. Let $\Pi_{1}=\Pi \times \operatorname{ker}(\tau)$. Thus, $\Pi_{1}$ is a pro- $p \Omega$-group and the natural projection map from $\Pi_{1} \rightarrow \Pi$ is a surjective $\Omega$-homomorphism. Hence, as we have shown above, there is a surjective $\Omega$-homomorphism $\sigma_{1}$ from $\Gamma$ to $\Pi_{1}$ such that $\widetilde{\sigma}_{1}=\tau_{1}$. Composing with the projection map from $\Pi_{1}$ to $\Pi$ gives a surjective $\Omega$-homomorphism $\sigma$ such that $\widetilde{\sigma}=\tau$.

Remark 2.3.2. Proposition 2.1.1 is a consequence of proposition 2.3.1. If $\Omega$ satisfies assumption A, then we pointed out at the end of section 2.3 that one can construct a free pro- $p \Omega$-group $\Gamma$ with any specified $\Omega$-type as a free product. The construction gives us a set of generators $\left\{\gamma_{1}, \ldots, \gamma_{n}\right\}$ of $\Gamma$ consisting of $\Omega$-elements. If $\chi$ is a $\mathbf{Z}_{p}^{\times}$-valued character of $\Omega$, then the number of distinguished generators which are $\chi$-elements for $\Omega$ is $m_{\chi}(\widetilde{\Gamma})$.

For any $\Pi$, we can construct $\Gamma$ so that $\Gamma$ has the same $\Omega$-type as $\Pi$. Furthermore, if we choose a basis $\left\{\widetilde{\pi}_{1}, \ldots ., \widetilde{\pi}_{n}\right\}$ of $\widetilde{\Pi}$-consisting of $\Omega$-elements, and if we suitably modify the indexing for the $\gamma_{i}^{\prime}$ 's, then we can choose $\tau: \widetilde{\Gamma} \rightarrow \widetilde{\Pi}$ so that $\tau\left(\widetilde{\gamma}_{i}\right)=\widetilde{\pi}_{i}$ for $1 \leq i \leq n$. If we choose a lifting $\sigma$ of $\tau$ as in proposition 2.3.1, then $\pi_{i}=\sigma\left(\gamma_{i}\right)$ will be an $\Omega$-element of $\Pi$ whose image in $\widetilde{\Pi}$ is $\widetilde{\pi}_{i}$ for each $i$. $\diamond$

Remark 2.3.3. This remark concerns the usual universal mapping property for a free pro- $p$ group $\Gamma$ on a finite set $\Sigma$. If one replaces $\Sigma$ by another set of topological generators $S$ which has the same cardinality as $\Sigma$, then the universal mapping property also holds for $S$ and $\Gamma$. To justify this, first note that if $f: \Sigma \rightarrow S$ is a bijection, then the unique continuous homomorphism $\varphi: \Gamma \rightarrow \Gamma$ such that $\left.\varphi\right|_{\Sigma}=f$ is a minimal presentation of $\Gamma$. Hence the number of relations is the $\mathbf{F}_{p}$-dimension of $H^{2}\left(\Gamma, \mathbf{F}_{p}\right)$, which is zero. Hence $\varphi$ is also injective and therefore is an isomorphism. The universal mapping property for the pair $(\Gamma, S)$ follows from that same property for the pair $(\Gamma, \Sigma)$.

\section{Properties of $p$-rational fields.}

Suppose that $K$ is a number field and that $p$ is an odd prime. Let $\Sigma_{p}$ be the set of primes of $K$ lying above $p$. We define the following three extensions of $K: M$ is the compositum of all finite $p$-extensions of $K$ which are unramified outside of $\Sigma_{p}, M^{a b}$ is the maximal abelian extension of $K$ contained in $M$, and $L$ is the compositum of all cyclic extensions of $K$ of degree $p$ which are contained in $M$. If we let $\Gamma$ denote $\operatorname{Gal}(M / K)$, then $\Gamma$ is a pro- $p$ group, $\Gamma^{a b} \cong \operatorname{Gal}\left(M^{a b} / K\right)$ is the maximal, abelian quotient of $\Gamma$, and the Frattini quotient $\widetilde{\Gamma}$ can be identified with $\operatorname{Gal}(L / K)$. If it is needed to avoid confusion, we will include a subscript $K$, writing $M_{K}$ instead of $M, \Gamma_{K}^{a b}$ instead of $\Gamma^{a b}$, etc.

We can consider $\Gamma^{a b}$ as a $\mathbf{Z}_{p}$-module. It is known to be finitely-generated. The Frattini quotients of $\Gamma$ and $\Gamma^{a b}$ are the same and that gives the first of the following inequalities:

$$
\operatorname{dim}_{\mathbf{F}_{p}}(\widetilde{\Gamma}) \geq \operatorname{rank}_{\mathbf{Z}_{p}}\left(\Gamma^{a b}\right) \geq r_{2}(K)+1
$$


Here $r_{2}(K)$ denotes the number of complex primes of $K$. The second inequality is a well-known result. (See [Was], section 13.5, for example.) Let $r_{1}(K)$ denote the number of real primes of $K$. If $K / \mathbf{Q}$ is Galois, then there are two possibilities: Either $r_{1}(K)=0, r_{2}(K)=\frac{1}{2}[K: \mathbf{Q}]$ (the totally complex case) or $r_{1}(K)=[K: \mathbf{Q}], r_{2}(K)=0$ (the totally real case).

A number field $K$ is said to be "p-rational" if $\operatorname{dim}_{\mathbf{F}_{p}}(\widetilde{\Gamma})=r_{2}(K)+1$. The simplest example is $K=\mathbf{Q}$. One can show by class field theory (or by using the Kronecker-Weber theorem) that $M=\mathbf{Q}_{\infty}$, the cyclotomic $\mathbf{Z}_{p}$-extension of $\mathbf{Q}$. In general, a number field $K$ is $p$-rational if and only if the following two requirements are satisfied:

(i) $\operatorname{rank}_{\mathbf{z}_{p}}\left(\Gamma^{a b}\right)=r_{2}(K)+1$,

(ii) $\Gamma^{a b}$ is torsion-free as a $\mathbf{Z}_{p}$-module.

The first statement is Leopoldt's conjecture for $K$ and $p$. It is known to hold when $K$ is an abelian extensions of $\mathbf{Q}$ and $p$ is any prime. In principle, one can check the second requirement by using class field theory. We will discuss this for various types of number fields in section 4 .

Consider the special case where $K$ is totally real. Then $K$ is $p$-rational if and only if $M^{a b}=$ $K \mathbf{Q}_{\infty}$, the cyclotomic $\mathbf{Z}_{p}$-extension of $K$. It then follows from BBT that $\Gamma$ has one topological generator and therefore that $\Gamma$ is abelian. Thus, $\Gamma=\Gamma^{a b} \cong \mathbf{Z}_{p}$, a free pro- $p$ group on one generator. That is, a totally real number field $K$ will be $p$-rational if and only if $M=K \mathbf{Q}_{\infty}$.

3.1. Freeness. The importance of $p$-rationality for us is contained in the following result which is proved in $[\mathrm{MoNg}]$. The conclusion is one of their equivalent statements about $p$-rationality. It will be useful to give an argument here.

Proposition 3.1.1. If $K$ is $p$-rational, then $\Gamma$ is a free pro-p group on $r_{2}(K)+1$ generators.

Proof. We have already explained this result if $r_{2}(K)=0$. In general, it turns out to be a consequence of the fact that the global Euler-Poincaré characteristic for the trivial $\mathrm{Gal}\left(K_{\Sigma} / K\right)$ module $\mathbf{Z} / p \mathbf{Z}$ is equal to $-r_{2}(K)$. Here $\Sigma$ consists of the primes lying over $p$ or $\infty$ and $K_{\Sigma}$ is the maximal extension of $K$ unramified outside of $\Sigma$. We obviously have $\operatorname{dim}_{\mathbf{F}_{p}}\left(H^{0}\left(K_{\Sigma} / K, \mathbf{Z} / p \mathbf{Z}\right)\right)=1$. Assuming that $K$ is $p$-rational, we have

$$
\operatorname{dim}_{\mathbf{F}_{p}}\left(H^{1}\left(K_{\Sigma} / K, \mathbf{Z} / p \mathbf{Z}\right)\right)=\operatorname{dim}_{\mathbf{F}_{p}}\left(H^{1}(\Gamma, \mathbf{Z} / p \mathbf{Z})\right)=r_{2}(K)+1
$$

Using the Euler-Poincaré characteristic, it follows that $H^{2}\left(K_{\Sigma} / K, \mathbf{Z} / p \mathbf{Z}\right)=0$. Furthermore, by definition, $M$ has no nontrivial Galois $p$-extension contained in $K_{\Sigma}$. A result of Neumann (corollary 10.4.3 in [NSW]) implies that $H^{i}\left(K_{\Sigma} / M, \mathbf{Z} / p \mathbf{Z}\right)=0$ for all $i \geq 1$. Using that result and proposition 1.6.6 in [NSW], one sees that the inflation map

$$
H^{i}(\Gamma, \mathbf{Z} / p \mathbf{Z}) \longrightarrow H^{i}\left(K_{\Sigma} / K, \mathbf{Z} / p \mathbf{Z}\right)
$$


is an isomorphism for any $i \geq 1$. In particular, we have $H^{2}(\Gamma, \mathbf{Z} / p \mathbf{Z})=0$. It follows that $\Gamma$ has a minimal presentation with $r_{2}(K)+1$ generators and no relations, proving the stated result.

Note that the converse of proposition 3.1.1 is clearly true. In fact, one has the following stronger statement: If $\Gamma$ is a free pro-p group, then $K$ is p-rational. To see this, note that (4) implies that the Euler-Poincaré characteristic for $\mathbf{Z} / p \mathbf{Z}$ as a $\Gamma$-module is also equal to $-r_{2}(K)$. In particular, if $\Gamma$ is a free pro- $p$ group, then $H^{2}(\Gamma, \mathbf{Z} / p \mathbf{Z})=0$ and hence the $\mathbf{F}_{p^{-}}$-dimension of $H^{1}(\Gamma, \mathbf{Z} / p \mathbf{Z})$ is $r_{2}(K)+1$. Therefore, $\Gamma$ indeed has a topological generating set of that cardinality.

Remark 3.1.2. There is a considerable literature concerning $p$-rational fields, including [Ngu], $[\mathrm{JaNg}],[\mathrm{Mov}]$, and $[\mathrm{MoNg}]$. One additional equivalent statement which is found in those references (e.g., proposition 2 in [Mov]) involves the subgroup $\mathcal{H}_{p}(K)$ of $K^{\times}$consisting of $p$-hyperprimary elements. An element $\alpha \in K^{\times}$is said to be "p-hyperprimary" if $\alpha \mathcal{O}_{K}=\mathfrak{a}^{p}$ for some fractional ideal $\mathfrak{a}$ of $K$ and if $\alpha \in\left(K_{v}^{\times}\right)^{p}$ for all primes $v$ of $K$ lying above $p$. Then $K$ is $p$-rational if and only if the following two statements are satisfied:

(a) The map $\mu(K)_{p} \longrightarrow \prod_{v \in \Sigma_{p}} \mu\left(K_{v}\right)_{p}$ is an isomorphism.

(b) $\mathcal{H}_{p}(K)=\left(K^{\times}\right)^{p}$.

In statement $(a), \mu(K)_{p}$ and $\mu\left(K_{v}\right)_{p}$ denote the groups of $p$-power roots of unity in the specified fields. It is obviously satisfied if $\mu_{p} \notin K_{v}$ for all $v \mid p$. If the class number of $K$ is not divisible by $p$, then statement (b) means that if a unit $\alpha$ of $K$ is a $p$-th power in the completions $K_{v}$ for all $v \in \Sigma_{p}$, then $\alpha$ is a $p$-th power in $K$ itself.

If $K$ fails to be $p$-rational, it might still be useful to know if there exists a Galois extension $N$ of $K$ such that $\operatorname{Gal}(N / K)$ is a free pro- $p$ group on $r$ generators for some reasonably large value of $r$. For our purpose, we would also want $N$ to be Galois over $\mathbf{Q}$. Very little is known about this question. Some comments can be found in [Yam] and [Hub].

3.2. The $\Omega$-type of $\Gamma=\operatorname{Gal}(M / K)$. Assume now that $K$ is Galois over $\mathbf{Q}$ and let $\Omega=\operatorname{Gal}(K / \mathbf{Q})$. It is clear that $M=M_{K}$ will also be Galois over $\mathbf{Q}$. We then have an exact sequence:

$$
1 \longrightarrow \Gamma \longrightarrow \operatorname{Gal}(M / \mathbf{Q}) \longrightarrow \Omega \longrightarrow 1 .
$$

We will assume from now on that $K$ is totally complex. If $K \rightarrow \mathbf{C}$ is a field embedding, then the restriction of complex conjugation to $K$ is an element of order 2. We let $\Omega_{\infty}$ denote the subgroup that it generates. It may depend on the embedding, but the choice won't matter.

There is a well-defined action of $\Omega$ on $\Gamma^{a b}$. The next result is valid even if $\Omega$ has order divisible by $p$. We let $\chi_{0}$ denote the trivial representation of $\Omega$. 
Proposition 3.2.1. Assume that $K$ is a totally complex Galois extension of $\mathbf{Q}$ and that Leopoldt's conjecture holds for $K$ and $p$. Let $\varepsilon_{1}$ denote the nontrivial character of $\Omega_{\infty}$. Then

$$
\Gamma^{a b} \otimes_{\mathbf{z}_{p}} \mathbf{Q}_{p} \cong \operatorname{Ind}_{\Omega_{\infty}}^{\Omega}\left(\varepsilon_{1}\right) \oplus \chi_{0}
$$

as representations spaces for $\Omega$.

Proof. The argument is based on class field theory. Let $V=\Gamma^{a b} \otimes \mathbf{z}_{p} \mathbf{Q}_{p}$. Note that $V$ is a $\mathbf{Q}_{p}$-representation space for $\Omega$ of dimension $r_{2}(K)+1$ since we assume that Leopoldt's conjecture holds for $K$ and $p$. Let $E$ denote the group of units of $K$. Then $W=E \otimes_{\mathbf{z}} \mathbf{Q}_{p}$ is another $\mathbf{Q}_{p^{-}}$ representation space for $\Omega$. Its dimension is $[K: \mathbf{Q}]-1$ if $K$ is real, $\frac{1}{2}[K: \mathbf{Q}]-1$ if $K$ is complex. It is well-known that

$$
W \oplus \chi_{0} \cong \operatorname{Ind}_{\Omega_{\infty}}^{\Omega}\left(\varepsilon_{0}\right)
$$

as representation spaces for $\Omega$, where $\varepsilon_{0}$ is the trivial character of $\Omega_{\infty}$. This can be deduced from the usual proof of Dirichlet's unit theorem. If we replace $E$ by a subgroup $E^{\prime}$ of finite index, then $E^{\prime} \otimes_{\mathbf{Z}} \mathbf{Q}_{p}$ defines an isomorphic representation space for $\Omega$.

Let $\mathcal{K}=K \otimes_{\mathbf{Q}} \mathbf{Q}_{p}$. We can identify $\mathcal{K}$ with the product of the completions of $K$ at the primes above $p$. Let $\mathcal{U}$ be the product of the local unit groups in the completions. Thus, $\mathcal{U}$ is a compact subgroup of $\mathcal{K}^{\times}$. Let $\mathcal{U}^{\prime}$ denote the maximal pro- $p$ subgroup of $\mathcal{U}$, which has finite index in $\mathcal{U}$. Then $\mathcal{U}^{\prime}$ can be regarded as a $\mathbf{Z}_{p}$-module. The log maps for the completions of $K$ at the primes above $p$ define a $\mathbf{Z}_{p}$-module homomorphism $\log _{\mathcal{U}^{\prime}}$ from $\mathcal{U}^{\prime}$ to $\mathcal{K}$ with finite kernel and open image. Now $\Omega$ acts on $\mathcal{K}$ as a group of $\mathbf{Q}_{p}$-algebra automorphisms. Regarding $\mathcal{K}$ as a $\mathbf{Q}_{p}$-representation space for $\Omega$, it is isomorphic to the regular representation. The action of $\Omega$ on the multiplicative group of $\mathcal{K}$ induces an action of $\Omega$ on $\mathcal{U}^{\prime}$. Furthermore, $\log _{\mathcal{U}^{\prime}}$ is $\Omega$-equivariant. It follows that the $\mathbf{Q}_{p}$-representation space $U=\mathcal{U}^{\prime} \otimes \mathbf{z}_{p} \mathbf{Q}_{p}$ for $\Omega$ is isomorphic to the regular representation.

There is a canonical embedding $E \rightarrow \mathcal{U}$. Let $E^{\prime}$ denote the maximal subgroup of $E$ which is mapped into $\mathcal{U}^{\prime}$ by that embedding. For simplicity, we identify $E^{\prime}$ with its image in $\mathcal{U}^{\prime}$. Then we get an induced map of $E^{\prime} \otimes_{\mathbf{Z}} \mathbf{Z}_{p} \rightarrow \mathcal{U}^{\prime}$ and the image of that map is the closure $\overline{E^{\prime}}$ of $E^{\prime}$ in $\mathcal{U}^{\prime}$. Since Leopoldt's conjecture is assumed to hold for $K$ and $p$, the $\mathbf{Z}_{p}$-ranks of $E^{\prime} \otimes_{\mathbf{z}} \mathbf{Z}_{p}$ and $\overline{E^{\prime}}$ are equal and therefore the kernel of the map $E^{\prime} \otimes_{\mathbf{Z}} \mathbf{Z}_{p} \rightarrow \overline{E^{\prime}}$ is finite. It follows that the representation spaces $E^{\prime} \otimes_{\mathbf{z}} \mathbf{Q}_{p}$ and $\overline{E^{\prime}} \otimes_{\mathbf{z}_{p}} \mathbf{Q}_{p}$ for $\Omega$ are isomorphic.

Class field theory defines a homomorphism

$$
\mathcal{U}^{\prime} / \overline{E^{\prime}} \longrightarrow \Gamma^{a b}
$$

which has finite kernel and cokernel. Tensoring those $\mathbf{Z}_{p}$-modules with $\mathbf{Q}_{p}$ defines an isomorphism of $\mathbf{Q}_{p}$-representation spaces for $\Omega$. It follows that $U / W \cong V$. We have already discussed the structure of $E^{\prime} \otimes \mathbf{z}_{p} \mathbf{Q}_{p}$, which is isomorphic to $W$, and of $U=\mathcal{U}^{\prime} \otimes \mathbf{z}_{p} \mathbf{Q}$. The regular representation of $\Omega_{\infty}$ is isomorphic to $\varepsilon_{0} \oplus \varepsilon_{1}$ and hence

$$
U \cong \operatorname{Ind}_{\Omega_{\infty}}^{\Omega}\left(\varepsilon_{0}\right) \oplus \operatorname{Ind}_{\Omega_{\infty}}^{\Omega}\left(\varepsilon_{1}\right)
$$


The stated isomorphism for $V$ follows from the above isomorphisms for $W$ and $U$.

Assume that $\Omega$ satisfies assumption B. The Schur-Zassenhaus theorem then implies that the exact sequence (5) splits and hence that there exists a splitting homomorphism from $\Omega$ to $\operatorname{Gal}(M / \mathbf{Q})$. It is not unique, but we will fix one choice. Thus, $\Omega$ can be identified with a $\operatorname{subgroup~of~} \operatorname{Gal}(M / \mathbf{Q})$. Conjugation by elements of $\Omega$ then defines an action of $\Omega$ on $\Gamma$, and hence $\Gamma$ becomes an $\Omega$-group. One sees easily that the $\Omega$-type of $\Gamma$ does not depend on the choice of splitting homomorphism. If one assumes that $K$ is $p$-rational, then $\Gamma$ is a free $\Omega$-group. As mentioned in remark 2.2.1, its $\Omega$-type is then determined by the representation space $V=\Gamma^{a b} \otimes \mathbf{z}_{p} \mathbf{Q}_{p}$ for $\Omega$. However, if $K$ is not $p$-rational, then the $\mathbf{Z}_{p}$-torsion submodule of $\Gamma^{a b}$ will be nontrivial and will make an additional contribution to the $\Omega$-type of $\Gamma$.

If $K$ is a totally complex, abelian extension of $\mathbf{Q}$ and $\chi \in \widehat{\Omega}$, then we say that $\chi$ is odd if $\left.\chi\right|_{\Omega_{\infty}}=\varepsilon_{1}$. Let $\widehat{\Omega}_{o d d}$ denote the set of odd characters of $\Omega$. The following corollary determines the $\Omega$-type of $\Gamma$ completely under the stated assumptions. It follows directly from remark 2.2 .1 and proposition 3.2.1.

Corollary 3.2.2. Suppose that $K$ is totally complex and p-rational. Suppose that $\Omega$ satisfies assumption A. Then $m_{\chi}(\widetilde{\Gamma})=1$ for all $\chi \in \widehat{\Omega}_{\text {odd }} \cup\left\{\chi_{0}\right\}$ and $m_{\chi}(\widetilde{\Gamma})=0$ for all other $\chi$ 's in $\widehat{\Omega}$. These multiplicities determine the $\Omega$-type of $\Gamma$.

Remark 3.2.3. If $\Omega_{\infty}$ is a normal subgroup of $\Omega$, then $K^{\Omega_{\infty}}$ is the maximal totally real subfield of $K$ and $K$ is a so-called CM field. As above, we let $V=\Gamma^{a b} \otimes \mathbf{z}_{p} \mathbf{Q}_{p}$. One has a decomposition $V=V^{\left(\varepsilon_{0}\right)} \oplus V^{\left(\varepsilon_{1}\right)}$ for the action of $\Omega_{\infty}$. Even without assuming the validity of Leopoldt's conjecture, the above proof shows that

$$
V^{\left(\varepsilon_{1}\right)} \cong \operatorname{Ind}_{\Omega_{\infty}}^{\Omega}\left(\varepsilon_{1}\right)
$$

as representation spaces for $\Omega$. If one doesn't assume that $\Omega_{\infty}$ is normal, then the proof shows that $\operatorname{Ind}_{\Omega_{\infty}}^{\Omega}\left(\varepsilon_{1}\right) \oplus \chi_{0}$ is a direct summand in $V$ as a representation space for $\Omega$.

We can also say something about $\widetilde{\Gamma}$ as an $\mathbf{F}_{p}$-representation space in the case where $\Omega$ has order prime to $p$. Assume that $K$ is totally complex. For brevity, let $\gamma$ and $\xi$ denote the $\mathbf{Q}_{p^{-}}$ representations of $\Omega$ defined by $V$ and by $\operatorname{Ind}_{\Omega_{\infty}}^{\Omega}\left(\varepsilon_{1}\right) \oplus \chi_{0}$, respectively. Since $\xi$ is a direct summand in $\gamma$, and $\Omega$ has order prime to $p$, it follows from remark 2.2.1 that $\bar{\xi}$ is a direct summand in $\bar{\gamma}$. Furthermore, if $\Gamma[p]$ denotes the maximal subgroup of $\Gamma$ of exponent $p$, then $\widetilde{\Gamma} \cong \bar{\gamma} \oplus \Gamma[p]$ as $\mathbf{F}_{p}$-representations spaces for $\Omega$. Therefore, it follows that $\bar{\xi}$ is a direct summand in $\widetilde{\Gamma}$.

3.3. Criteria involving subfields. Assume that $K / \mathbf{Q}$ is a finite, abelian extension and that $\Omega$ has order prime to $p$, but not necessarily exponent dividing $p-1$. Let $\operatorname{Irr}_{\mathbf{Q}_{p}}(\Omega)$ denote the set of irreducible representations of $\Omega$ over $\mathbf{Q}_{p}$, up to isomorphism. If $\chi \in \operatorname{Irr}_{\mathbf{Q}_{p}}(\Omega)$, we denote its degree by $n(\chi)$. Let $F_{\chi}$ be the fixed field for $\operatorname{ker}(\chi)$. Then $F_{\chi}$ is a cyclic extension of $\mathbf{Q}$. Note that $n(\chi)=1$ if and only if $\left[F_{\chi}: \mathbf{Q}\right]$ divides $p-1$. Also, if $F$ is any extension of $\mathbf{Q}$ contained in $K$, then 
$F_{\chi} \subseteq F$ if and only if $\chi$ factors through $\operatorname{Gal}(F / \mathbf{Q})$. If $F / \mathbf{Q}$ is cyclic, then there exists at least one $\chi \in \operatorname{Irr}_{\mathbf{Q}_{p}}(\Omega)$ such that $F_{\chi}=F$.

We have the following canonical decomposition, where we let $e_{\chi} \in \mathbf{Z}_{p}[\Omega]$ denote the idempotent for $\chi$ and where our notation now includes a subscript indicating the field.

$$
\Gamma_{K}^{a b} \cong \bigoplus_{\chi}\left(\Gamma_{K}^{a b}\right)^{e_{\chi}}
$$

as $\mathbf{Z}_{p}[\Omega]$-modules, where $\chi$ varies over $\operatorname{Irr}_{\mathbf{Q}_{p}}(\Omega)$ in the above direct sum. Furthermore, if $F$ is any subfield of $K$, cyclic or not, then $\Gamma_{F}^{a b}$ can be identified with the maximal quotient of $\Gamma_{K}^{a b}$ on which $\operatorname{Gal}(K / F)$ acts trivially. Hence, we have the following isomorphism

$$
\Gamma_{F}^{a b}=\operatorname{Gal}\left(M_{F}^{a b} / F\right) \cong \bigoplus_{F_{\chi} \subseteq F}\left(\Gamma_{K}^{a b}\right)^{e_{\chi}}
$$

where the notation indicates that $\chi$ varies over the elements of $\operatorname{Irr}_{\mathbf{Q}_{p}}(\Omega)$ such that $F_{\chi} \subseteq F$. In particular, taking $F=\mathbf{Q}$, we have $M_{\mathbf{Q}}^{a b}=\mathbf{Q}_{\infty}$, the cyclotomic $\mathbf{Z}_{p}$-extension of $\mathbf{Q}$. This is so because $p$ is odd. Thus, we have $\left(\Gamma_{K}^{a b}\right)^{e} \chi_{0} \cong \operatorname{Gal}\left(\mathbf{Q}_{\infty} / \mathbf{Q}\right) \cong \mathbf{Z}_{p}$, where $\chi_{0}$ denotes the trivial character.

The above remark gives a proof of the following proposition. One just observes that $\Gamma_{K}^{a b}$ is torsion-free if and only if $\left(\Gamma_{K}^{a b}\right)^{e_{\chi}}$ is torsion-free for all $\chi \in \widehat{\Omega}$.

Proposition 3.3.1. If $K$ is a finite abelian extension of $\mathbf{Q}$ and $[K: \mathbf{Q}]$ is not divisible by $p$, then $K$ is p-rational if and only if every cyclic extension of $\mathbf{Q}$ contained in $K$ is p-rational.

Remark 3.3.2. Let us assume that $K$ satisfies assumption $\mathbf{A}$, but not necessarily that $K$ is $p$ rational. We let $\widehat{\Omega}_{\text {odd }}$ denote the set of odd characters of $\Omega$, which we can regard as characters with values in $\mathbf{Z}_{p}^{\times}$. They can also be regarded as irreducible representations for $\Omega$ over $\mathbf{Q}_{p}$. Remarks 3.2.3 implies that the $\mathbf{Z}_{p}$-rank of $\left(\Gamma_{K}^{a b}\right)^{e}$ is equal to 1 if $\chi \in \widehat{\Omega}_{\text {odd }} \bigcup\left\{\chi_{0}\right\}$. Thus, for every such $\chi$, there exists a uniquely determined Galois extension $K_{\infty}^{(\chi)}$ of $K$ with the following properties: $\operatorname{Gal}\left(K_{\infty}^{(\chi)} / K\right) \cong \mathbf{Z}_{p}, K_{\infty}^{(\chi)}$ is Galois over $\mathbf{Q}$, and $\Omega$ acts on $\operatorname{Gal}\left(K_{\infty}^{(\chi)} / K\right)$ by the character $\chi$. The field $K_{\infty}^{(\chi)}$ is a $\mathbf{Z}_{p}$-extension of $K$. Using the notation of section 2 , we have $\operatorname{Gal}\left(K_{\infty}^{(\chi)} / \mathbf{Q}\right) \cong \Gamma_{\chi} \rtimes \Omega$. Note that $K_{\infty}^{\left(\chi_{0}\right)}=K \mathbf{Q}_{\infty}$, the cyclotomic $\mathbf{Z}_{p}$-extension of $K$. The field $M_{K}^{a b}$ is a finite extension of the compositum of the $K_{\infty}^{(\chi)}$ 's. The field $K$ will be $p$-rational if and only if $M_{K}^{a b}$ coincides with that compositum.

We will mention without proof a criterion which requires only assumption $\mathbf{B}$ for $\Omega$. If $T$ is a collection of subgroups of $\Omega$, then we say that $T$ is "ample" if the following property holds: For every $\mathbf{Q}_{p}$-irreducible representation $\chi$ of $\Omega, \mathbf{1}_{\Theta}$ is a constituent in $\left.\chi\right|_{\Theta}$ for at least one $\Theta \in T$. 
Here $\mathbf{1}_{\Theta}$ denotes the trivial representation of $\Theta$. Equivalently, one can make the same requirement on the restrictions $\left.\chi\right|_{\Theta}$, where $\chi$ varies over all the absolutely irreducible representations of $\Omega$. If $F$ is any subfield of $K$, let $\Theta_{F}=\operatorname{Gal}(K / F)$. A collection $\mathcal{F}$ of subfields of $K$ is said to be ample if the corresponding collection of subgroups $\left\{\Theta_{F}\right\}_{F \in \mathcal{F}}$ is ample. The proof of the next result is somewhat similar to the proof of proposition 3.3.1. It is not difficult if $K$ is totally real. If $K$ is totally complex, then remark 3.2.3 is useful as well as the following easily proved fact:

$$
\operatorname{dim}_{\mathbf{Q}_{p}}\left(\operatorname{Ind}_{\Omega_{\infty}}^{\Omega}\left(\varepsilon_{\infty}\right)^{\Theta_{F}}\right)=r_{2}(F)
$$

for every subfield $F$ of $K$. If $\Omega$ is abelian, then the collection $\mathcal{F}$ consisting of all cyclic extensions of $\mathbf{Q}$ contained in $K$ will obviously be ample. Thus, the following result is a generalization of proposition 3.3.1.

Proposition 3.3.3. Suppose that $K / \mathbf{Q}$ is a finite Galois extension and that $\mathcal{F}$ is an ample collection of subfields of $K$. Suppose that $p$ is a prime and that $[K: \mathbf{Q}]$ is not divisible by $p$. Then $K$ is p-rational if and only if every field $F$ in $\mathcal{F}$ is p-rational.

As one simple illustration, suppose that $\operatorname{Gal}(K / \mathbf{Q}) \cong S_{3}$ and that $p>3$. One can take $\mathcal{F}$ to consist of the quadratic subfield and any cubic subfield of $K$. One sees easily that $\mathcal{F}$ is ample.

\section{Examples of abelian $p$-rational fields.}

We continue to assume that $p$ is an odd prime. We describe a variety of examples of $p$-rational fields which will be useful in the construction in section 6 . We have already mentioned the simple example $K=\mathbf{Q}$ for which we have $M_{\mathbf{Q}}^{a b}=\mathbf{Q}_{\infty}$, the cyclotomic $\mathbf{Z}_{p}$-extension of $\mathbf{Q}$.

4.1. Quadratic fields. Suppose that $[K: \mathbf{Q}]=2$. Then $\widehat{\Omega}=\left\{\chi_{0}, \chi_{1}\right\}$, where $\chi_{0}$ is the trivial character and $\chi_{1}$ is of order 2 . If $K$ is complex, then $\chi_{1}$ is odd and the field $K_{\infty}^{\left(\chi_{1}\right)}$ defined in remark 3.3.2 is usually called the anticyclotomic $\mathbf{Z}_{p}$-extension of $K$. The field $K_{\infty}^{\left(\chi_{0}\right)}$ is just $K \mathbf{Q}_{\infty}$, the cyclotomic $\mathbf{Z}_{p}$-extension of $K$. Let $h_{K}$ denote the class number of $K$. The following result is not entirely new. Very similar results are proved in [Fuj] and in [Min] if $K$ is complex. For simplicity, we make an assumption about $p$ which guarantees that $\mu_{p} \not \subset K_{v}$ for the primes(s) $v$ of $K$ lying over $p$.

Proposition 4.1.1. Suppose that $K$ is a quadratic field and that either $p \geq 5$ or that $p=3$ and is unramified in $K / \mathbf{Q}$.

(i) Assume that $K$ is complex. Then $K$ is p-rational if and only if the p-Hilbert class field of $K$ is contained in $K_{\infty}^{\left(\chi_{1}\right)}$. In particular, if $h_{K}$ is not divisible by $p$, then $K$ is p-rational. 
(ii) Assume that $K$ is real. Then $K$ is p-rational if and only if $h_{K}$ is not divisible by $p$ and the fundamental unit $\varepsilon_{0}$ of $K$ is not a p-th power in the completion $K_{v}$, where $v$ is a prime of $K$ lying above $p$.

Proof. The field $K$ will be $p$-rational if and only if $\left(\Gamma_{K}^{a b}\right)^{e_{\chi_{1}}}$ is torsion-free. We will let $\left(\Gamma_{K}^{a b}\right)_{\text {tors }}^{e_{\chi_{1}}}$ denote the torsion-subgroup of $\left(\Gamma_{K}^{a b}\right)^{e_{\chi_{1}}}$. The assumption about $p$ guarantees that the completion $K_{v}$ at a prime $v \mid p$ doesn't contain $\mu_{p}$, the group of $p$-th roots of unity. In the notation of the proof of proposition 3.2.1, one then has $\left(\mathcal{U}^{\prime}\right)^{e_{\chi_{1}}} \cong \mathbf{Z}_{p}$.

If $K$ is complex, then class field theory gives an exact sequence

$$
1 \longrightarrow\left(\mathcal{U}^{\prime}\right)^{e_{\chi_{1}}} \longrightarrow\left(\Gamma_{K}^{a b}\right)^{e_{\chi_{1}}} \longrightarrow \operatorname{Gal}(H / K) \longrightarrow 1
$$

where $H$ denotes the $p$-Hilbert class field of $K$. The image of $\left(\mathcal{U}^{\prime}\right)^{e_{\chi_{1}}}$ in $\left(\Gamma_{K}^{a b}\right)^{e_{\chi_{1}}}$ is just the inertia subgroup for the prime(s) $v$ dividing $p$. The surjectivity of the map to $\operatorname{Gal}(H / K)$ follows from the fact that $\Omega=\operatorname{Gal}(K / \mathbf{Q})$ acts on $\operatorname{Gal}(H / K)$ by $\chi_{1}$. It follows that $\left(\Gamma_{K}^{a b}\right)_{\text {tors }}^{e_{\chi_{1}}}$ is mapped injectively into $\operatorname{Gal}(H / K)$ under the restriction map. That image will be $\operatorname{Gal}\left(H / H^{\prime}\right)$, where $H^{\prime}$ is an extension of $K$ contained in $H$. Furthermore, the fixed field for $\left(\Gamma_{K}^{a b}\right)_{\text {tors }}^{e_{\chi_{1}}}$ is precisely $K_{\infty}^{\left(\chi_{1}\right)}$. Consequently, $H^{\prime}=H \cap K_{\infty}^{\left(\chi_{1}\right)}$. This shows that $\left(\Gamma_{K}^{a b}\right)^{e_{\chi_{1}}}$ is torsion-free if and only if $H \subset K_{\infty}^{\left(\chi_{1}\right)}$.

Now assume that $K$ is real. There is a power $\varepsilon=\varepsilon_{0}^{a}$, where $a$ is not divisible by $p$, such that $\varepsilon \in \mathcal{U}^{\prime}$. We can choose $a$ to be even so that $\varepsilon$ has norm 1 . Then $\varepsilon \in\left(\mathcal{U}^{\prime}\right)^{e_{\chi_{1}}}$ and we have an exact sequence

$$
1 \longrightarrow\left(\mathcal{U}^{\prime}\right)^{e_{\chi_{1}}} / \overline{\langle\varepsilon\rangle} \longrightarrow\left(\Gamma_{K}^{a b}\right)^{e_{\chi_{1}}} \longrightarrow \operatorname{Gal}(H / K) \longrightarrow 1
$$

Thus, $\left(\Gamma_{K}^{a b}\right)^{e_{\chi_{1}}}$ is finite if $K$ is real. It follows that $K$ is $p$-rational if and only if both $\left(\mathcal{U}^{\prime}\right)^{e_{\chi_{1}} / \overline{\langle\varepsilon\rangle}}$ and $\operatorname{Gal}(H / K)$ are trivial. The first group is nontrivial if and only if $\varepsilon$ is a $p$-th power in $\left(\mathcal{U}^{\prime}\right)^{e} \chi_{1}$, or equivalently, if and only if $\varepsilon_{0}$ is a $p$-th power in $K_{v}$ for $v \mid p$. The group $\operatorname{Gal}(H / K)$ is nontrivial if and only if $h_{K}$ is divisible by $p$.

The following corollaries deal with various special cases. The first two concern $p=3$ and $p=5$.

Corollary 4.1.2. Suppose that $K=\mathbf{Q}(\sqrt{d})$, where $d$ is a squarefree integer and $3 \nmid d$. Then $K$ is 3 -rational if and only if the class number of $\mathbf{Q}(\sqrt{-3 d})$ is not divisible by 3 .

The same criterion is also proved by Fujii in [Fuj] (theorem 4.1) and by Minardi in [Min] (the corollary to proposition 6.B) when $d<0$. One can weaken the assumption that $3 \nmid d$. It suffices to assume that $\mu_{3}$ is not contained in the completion $K_{v}$ of $K$ at a prime $v$ dividing 3 .

Proof. Take $p=3$. Let $L^{\left(\chi_{1}\right)}$ be the fixed field for the Frattini subgroup of $\left(\Gamma_{K}^{a b}\right)^{e_{\chi_{1}}}$. Thus, $L^{\left(\chi_{1}\right)}$ is Galois over $\mathbf{Q}, \operatorname{Gal}\left(L^{\left(\chi_{1}\right)} / K\right)$ is an abelian group of exponent 3, and $\operatorname{Gal}(K / \mathbf{Q})$ acts on $\operatorname{Gal}\left(L^{\left(\chi_{1}\right)} / K\right)$ by $\chi_{1}$. If $K$ is complex, then $L^{\left(\chi_{1}\right)}$ contains the first layer $K_{1}^{\left(\chi_{1}\right)}$ in the anticyclotomic 
$\mathbf{Z}_{3}$-extension $K_{\infty}^{\left(\chi_{1}\right)} / K$, which is a cyclic extension of $K$ of degree 3 . In that case, the field $K$ is 3 -rational if and only if $L^{\left(\chi_{1}\right)}=K_{1}^{\left(\chi_{1}\right)}$. If $K$ is real, then $K$ is 3-rational if and only if $L^{\left(\chi_{1}\right)}=K$.

We will use the reflection principle. We assume that $d \neq 1$ so that $[K: \mathbf{Q}]=2$. Let $J=$ $K\left(\mu_{3}\right)=\mathbf{Q}(\sqrt{d}, \sqrt{-3 d})$ and let $F=\mathbf{Q}(\sqrt{-3 d})$. Then a Kummer theory argument shows that $J L^{\left(\chi_{1}\right)}$ is a compositum of fields of the form $J(\sqrt[3]{\alpha})$, where $\alpha \in F^{\times}$and $N_{F / \mathbf{Q}}(\alpha)=1$. Since $3 \nmid d$, it is clear that 3 is ramified in $F / \mathbf{Q}$. It follows that $\operatorname{ord}_{v}(\alpha)=0$ for the prime $v$ of $F$ lying over 3. Furthermore, the fact that $L^{\left(\chi_{1}\right)} / K$ is unramified at primes $v$ not lying over 3 implies that $\operatorname{ord}_{v}(\alpha) \equiv 0(\bmod 3)$ for all such $v$. Thus, $(\alpha)=\mathfrak{a}^{3}$, where $\mathfrak{a}$ is a fractional ideal of $F$.

Assume that the class number of $F$ is not divisible by 3. Then $\mathfrak{a}$ is principal. Thus $\alpha=\beta^{3} \varepsilon$, where $\beta \in F^{\times}$and $\varepsilon$ is a unit of $F$. Thus, $J(\sqrt[3]{\alpha})=J(\sqrt[3]{\varepsilon})$. If $K$ is real, then $F$ is complex and $\varepsilon$ is a root of unity whose order is not divisible by 3 (because $d \neq 1$ ). Thus, $J(\sqrt[3]{\varepsilon})=J$ and hence $L^{\left(\chi_{1}\right)}=K$. If $K$ is complex, then $F$ is real and $\pm \varepsilon$ is a power of the fundamental unit of $F$. Hence $J(\sqrt[3]{\varepsilon})$ is a cyclic extension of $J$. It follows that $L^{\left(\chi_{1}\right)} / K$ is cyclic and consequently we must have $L^{\left(\chi_{1}\right)}=K_{1}^{\left(\chi_{1}\right)}$. In both cases, we see that $K$ is indeed 3-rational.

Conversely, assume that the class number of $F$ is divisible by 3 . Let $c$ be an ideal class of order 3. Then $\operatorname{Gal}(K / \mathbf{Q})$ acts on $c$ by $\chi_{1}$. One verifies easily that one can choose an ideal $\mathfrak{a} \in c$ so that $\operatorname{Gal}(K / \mathbf{Q})$ acts on $\mathfrak{a}$ by $\chi_{1}$ and that $\mathfrak{a}^{3}$ has a generator $\alpha$ such that $N_{K / \mathbf{Q}}(\alpha)=1$. Furthermore, $J(\sqrt[3]{\alpha}) / J$ has degree 3 and is unramified except at 3. It follows that $J L^{\left(\chi_{1}\right)}$ contains $J(\sqrt[3]{\alpha})$. If $K$ is real, then it follows that $L^{\left(\chi_{1}\right)} \neq K$ and therefore $K$ is not 3-rational. Now assume that $K$ is complex. Then $F$ is real and $J L^{\left(\chi_{1}\right)}$ also contains $J\left(\sqrt[3]{\varepsilon_{0}}\right)$, where $\varepsilon_{0}$ is the fundamental unit of $F$. It follows that $J L^{\left(\chi_{1}\right)} / J$ is not cyclic and hence $L^{\left(\chi_{1}\right)}$ is not a cyclic extension of $K$. This means that $K$ is not 3 -rational.

Remark 4.1.3. A similar argument gives the following generalization for an arbitrary odd prime $p$. We assume that $K=\mathbf{Q}(\sqrt{d})$ and that $p \nmid d$. Let $J=K\left(\mu_{p}\right)$ and let $A$ denote the maximal elementary abelian $p$-subgroup of the ideal class group of $J$. We regard $A$ as an $\mathbf{F}_{p \text {-representation }}$ space for $\operatorname{Gal}(J / \mathbf{Q})$. The action of $\operatorname{Gal}(J / \mathbf{Q})$ on $\mu_{p}$ is described by a character $\omega: \operatorname{Gal}(J / \mathbf{Q}) \rightarrow \mathbf{F}_{p}^{\times}$. We regard $\chi_{1}$ as an $\mathbf{F}_{p}^{\times}$-valued character of $\operatorname{Gal}(J / \mathbf{Q})$ too. Then the field $K$ is $p$-rational if and only if the $\omega \chi_{1}$-component $e_{\omega \chi_{1}} A$ of $A$ is trivial.

Corollary 4.1.4. Suppose that $K=\mathbf{Q}(\sqrt{d})$, where $d$ is squarefree and $d>1$. Assume that $d \equiv \pm 1$ $(\bmod 5)$. Let $\varepsilon_{0}=a_{0}+b_{0} \sqrt{d}$ be the fundamental unit of $K$, where $a_{0}, b_{0} \in \mathbf{Q}$ and have denominator 1 or 2 . Then $\operatorname{ord}_{5}\left(a_{0} b_{0}\right) \geq 1$. The field $K$ is 5 -rational if and only if $5 \nmid h_{K}$ and ord $_{5}\left(a_{0} b_{0}\right)=1$.

Proof. The congruence for $d$ means that 5 splits in $K / \mathbf{Q}$. The fact that either $a_{0}$ or $b_{0}$ is divisible by 5 , but not both, follows from the equation $a_{0}^{2}-b_{0}^{2} d= \pm 1$ and the congruence for $d$. Divisibility refers to the ring $\mathbf{Z}\left[\frac{1}{2}\right]$. If $\mathfrak{p}$ is either one of the two primes of $K$ dividing 5 , then $\varepsilon_{0}^{2} \equiv \pm 1(\bmod \mathfrak{p})$. It is clear that $\varepsilon_{0}$ is a 5 -th power in $K_{\mathfrak{p}}$ if and only if $\varepsilon_{0}^{2} \equiv \pm 1\left(\bmod \mathfrak{p}^{2}\right)$. Furthermore, it is not difficult to show $\varepsilon_{0}^{2} \equiv \pm 1\left(\bmod \mathfrak{p}^{2}\right)$ if and only if $a_{0}$ or $b_{0}$ is divisible by $5^{2}$. The stated result then follows from proposition 4.1.1. 
Corollary 4.1.5. Let $K=\mathbf{Q}(\sqrt{5})$. Suppose that $p$ is an odd prime and $p \neq 5$. We will let $q=p$ if $p \equiv \pm 1(\bmod 5)$ and $q=p^{2}$ if $p \equiv \pm 2(\bmod 5)$. Then $K$ is $p$-rational if and only if $F_{q} \not \equiv 1$ $\left(\bmod p^{2}\right)$, where $F_{q}$ is the q-th Fibonacci number.

Proof. Since $h_{K}=1$, it follows that $K$ is $p$-rational if and only if the fundamental unit $\varepsilon_{0}$ is not a $p$-th power in the completion of $K$ at a prime $\mathfrak{p}$ above $p$. Let $F_{n}$ denote the $n$-th Fibonacci number. One has a well-known formula $F_{n}=a \varepsilon_{0}^{n-1}+b \bar{\varepsilon}_{0}{ }^{n-1}$ for all $n \geq 1$, where $a, b \in K$ and $\bar{\varepsilon}$ is the conjugate of $\varepsilon$ in $K$. We have $\sqrt{5} a=\varepsilon_{0}$ and $\sqrt{5} b=-\bar{\varepsilon}_{0}$. Thus, $a$ and $b$ are units in $K_{\mathfrak{p}}$. Now $\varepsilon_{0}^{q-1} \equiv 1(\bmod \mathfrak{p})$ and $\varepsilon_{0}$ is a $p$-th power in $K_{\mathfrak{p}}$ if and only if $\varepsilon_{0}^{q-1} \equiv 1\left(\bmod \mathfrak{p}^{2}\right)$. Since $\bar{\varepsilon}_{0}=-\varepsilon_{0}^{-1}$, the above congruences for $\varepsilon_{0}$ give similar congruences for $\bar{\varepsilon}_{0}$. It follows that $F_{q} \equiv 1(\bmod p)$ and that $\varepsilon_{0}$ is a $p$-th power in $K_{\mathfrak{p}}$ if and only if $F_{q} \equiv 1\left(\bmod p^{2}\right)$, proving the stated result.

Corollary 4.1.6. Suppose that $K$ is an imaginary quadratic field, that $p$ satisfies the hypothesis in proposition 4.1.1, that the class number of $K$ is divisible by $p$, and that the p-primary subgroup of the ideal class group of $K$ is cyclic. Suppose that $\mathfrak{a}$ is a fractional ideal of $K$ whose ideal class has order $p$ and that $\alpha$ is a generator of $\mathfrak{a}^{p}$. Then $K$ is $p$-rational if and only if $\alpha$ is not a $p$-th power in $K_{v}$ for a prime $v$ of $K$ lying above $p$.

One can find this result in [Min], proposition 6.A. Note that if the $p$-primary subgroup of the class group of $K$ is not cyclic, then proposition 4.1.1 clearly implies that $K$ is not $p$-rational.

Proof. We will use the criterion in remark 3.1.1. The unit group of $K$ is finite and of order prime to $p$. Furthermore, $N_{K / \mathbf{Q}}(\alpha)$ is a $p$-th power in $\mathbf{Q}$. If there are two primes of $K$ above $p$ and if $\alpha$ is a $p$-th power in the completion of $K$ for one of those primes $v$, then $\alpha$ is also a $p$-th power in the completion at the other prime above $p$. The assumptions imply that if $\beta \in \mathcal{H}_{p}(K)$, the group of hyperprimary elements of $K$, then $\beta=\alpha^{i} \gamma^{p}$, where $\gamma \in K^{\times}$and $0 \leq i<p$. Furthermore, by definition, $\beta$ is a $p$-th power in $K_{v}$ for all $v \mid p$. The assumption about $p$ implies that $\mu\left(K_{v}\right)$ is trivial for $v \mid p$. Now if $\alpha$ is not a $p$-th power in $K_{v}$ for $v \mid p$, then $i=0$. Thus, it follows that $\mathcal{H}_{p}(K)=\left(K^{\times}\right)^{p}$ and hence that $K$ is $p$-rational. For the converse, note that $\alpha \notin\left(K^{\times}\right)^{p}$ and that if $\alpha$ is a $p$-th power in $K_{v}$ for a prime $v \mid p$, then $\alpha \in \mathcal{H}_{p}(K)$.

Remark 4.1.7. Suppose that $K$ is an imaginary quadratic field and that the hypothesis in proposition 4.1 .1 concerning the prime $p$ is satisfied. Let $A$ denote the $p$-primary subgroup of the ideal class group of $K$. The Artin map defines an isomorphism $A \rightarrow \operatorname{Gal}(H / K)$, where $H$ is the $p$-Hilbert class field of $K$. Let $H^{\prime}=H \cap K_{\infty}^{\left(\chi_{1}\right)}$. Let $B$ denote the inverse image of $\operatorname{Gal}\left(H / H^{\prime}\right)$ under the Artin map. Just as in the proof of part (a) of proposition 4.1.1, one sees that the restriction map gives an isomorphism of the torsion subgroup of $\Gamma_{K}^{a b}$ to $\operatorname{Gal}\left(H / H^{\prime}\right)$. Thus, $K$ is $p$-rational if and only if $B$ is trivial.

Corollary 4.1.6 then follows immediately from the following intrinsic description of $B$. Suppose that $\mathfrak{a}$ is a fractional ideal of $K$ and that the class $a=\operatorname{cl}(\mathfrak{a})$ is in $A$. Thus $\mathfrak{a}^{p^{t}}=\alpha \mathcal{O}_{K}$ for some $t \geq 0$ 
and some $\alpha \in K^{\times}$. We will say that $\mathfrak{a}$ is a "singular ideal" and that $a$ is a "singular class" if the following condition is satisfied: A generator $\alpha$ for $\mathfrak{a}^{p^{t}}$ is a $p^{t}$-th power in $K_{v}^{\times}$for all $v$ dividing $p$. It is easy to verify that this definition depends only on the ideal class $a$ and not on the choice of $\mathfrak{a}, \alpha$, or $t$. The set of singular classes in $A$ is obviously a subgroup of $A$. This subgroup is precisely $B$. It is not difficult to prove this by using the description of $\Gamma_{K}^{a b}$ given by class field theory. The ray class formulation is the most convenient. We omit the details.

4.2. Compositums of quadratic fields. Suppose now that $\Omega=\operatorname{Gal}(K / \mathbf{Q}) \cong(\mathbf{Z} / 2 \mathbf{Z})^{t}$ for some $t \geq 2$. If $\chi \in \widehat{\Omega}$, and $\chi \neq \chi_{0}$, then $\operatorname{ker}(\chi)$ is a subgroup of $\Omega$ of index 2 . We let $F_{\chi}$ denote the corresponding quadratic extension of $\mathbf{Q}$. All the quadratic subfields of $K$ are of the form $F_{\chi}$ for some $\chi \neq \chi_{0}$. There are $2^{t}-1$ such subfields. Proposition 3.3.1 shows that $K$ is $p$-rational if and only if all of the quadratic field $F_{\chi}$ 's are $p$-rational. It seems reasonable to make the following conjecture, although our numerical evidence is not very strong.

Conjecture 4.2.1. For any odd prime $p$ and for any $t$, there exists a $p$-rational field $K$ such that $\operatorname{Gal}(K / \mathbf{Q}) \cong(\mathbf{Z} / 2 \mathbf{Z})^{t}$

We can merely give a few examples. For $p=3$, we use corollary 4.1.2 to check 3-rationality. For $p=5$, we use corollary 4.1.4 for the real quadratic subfields of $K$. For the imaginary quadratic subfields, we can just check either that the class number is not divisible by 5 or we can use corollary 4.1.6 if the class number is divisible by 5 .

$$
\begin{array}{ll}
p=3, t=5: & K=\mathbf{Q}(\sqrt{-1}, \sqrt{2}, \sqrt{5}, \sqrt{11}, \sqrt{97}) \\
p=3, t=6: & K=\mathbf{Q}(\sqrt{-1}, \sqrt{13}, \sqrt{145}, \sqrt{209}, \sqrt{269}, \sqrt{373}) \\
p=5, t=5: & K=\mathbf{Q}(\sqrt{-1}, \sqrt{6}, \sqrt{11}, \sqrt{14}, \sqrt{59})
\end{array}
$$

The example for $p=3$ and $t=5$ was found by Sourav Sen Gupta. The example for $p=3$ and $t=6$ was found by Robert Bradshaw. In both cases, a number of examples were found, although only a very small proportion of the trial set. The example for $p=5$ was found by the author. The verification requires using the criterion in corollary 4.1.6 for just one of the imaginary quadratic subfields of $K$, namely $F=\mathbf{Q}(\sqrt{-11 \cdot 59})$.

4.3. The field $K=\mathbf{Q}\left(\mu_{p}\right)$. Under the assumption that $p$ is a regular prime, Shafarevich proved that $\Gamma_{K}$ is a free pro- $p$ group if $K=\mathbf{Q}\left(\mu_{p}\right)$. One finds this on page 139 in [Sha], an illustration of general results in that paper about generators and relations for certain Galois groups. Using the results about $p$-rationality cited in section 3 , we can give a rather short argument.

Proposition 4.3.1. Let $K=\mathbf{Q}\left(\mu_{p}\right)$. Suppose that $p$ is a regular prime. Then $K$ is a p-rational field. 
Proof. We are assuming that the class group of $K$ has no elements of order $p$. Let $\varepsilon_{1}, \ldots, \varepsilon_{r}$ be a fundamental set of units for $K$, where $r=r_{2}(K)-1$. Let $\zeta_{p}$ be a generator for $\mu_{p}$. Then a straightforward Kummer theory argument shows that

$$
L=K\left(\sqrt[p]{p}, \sqrt[p]{\zeta_{p}}, \sqrt[p]{\eta_{1}}, \ldots, \sqrt[p]{\eta_{r}}\right)
$$

where $L$ is the fixed field for $\Phi\left(\Gamma_{K}\right)$. Consequently, one sees that

$$
\operatorname{dim}_{\mathbf{F}_{p}}(\operatorname{Gal}(L / K))=r+2=r_{2}(K)+1 .
$$

It follows that the inequalities (3) are equalities. Therefore $K$ is indeed a $p$-rational field.

4.4. The field $K=\mathbf{Q}\left(\mu_{5}\right)$ and $p \neq 5$. It is known that the class number of $K$ is 1 . Also, $\mu_{p}$ is not contained in the completion of $K$ at the primes lying above $p$. It follows that $\left(\Gamma_{K}^{a b}\right)^{e_{\chi}}$ is torsion-free when $\chi$ is a faithful, irreducible representation of $\Omega=\operatorname{Gal}(K / \mathbf{Q})$. Such a representation $\chi$ is odd. If $p \equiv 1(\bmod 4)$, then there are two such $\chi$ 's, both 1-dimensional over $\mathbf{Q}_{p}$, but if $p \equiv 3(\bmod 4)$, then there is just one such $\chi$, a 2-dimensional representation over $\mathbf{Q}_{p}$. One then sees from the proof of proposition 3.3.1 that $K=\mathbf{Q}\left(\mu_{5}\right)$ will be $p$-rational if and only if $\left(\Gamma_{K}^{a b}\right)^{e_{\chi}}$ is torsion-free when $\chi$ is the character of $\Omega$ of order 2 , which in turn means that the field $\mathbf{Q}(\sqrt{5})$ is $p$-rational. Corollary 4.1.5 gives a useful criterion involving Fibonacci numbers. Thus, we see that $K=\mathbf{Q}\left(\mu_{5}\right)$ is $p$-rational if and only if $F_{q} \not \equiv 1\left(\bmod p^{2}\right)$, where $q=p$ if $p$ is a quadratic residue modulo $5, q=p^{2}$ if $p$ is a quadratic nonresidue modulo 5 .

We have searched for primes $p$ for which $K$ fails to be $p$-rational. The criterion just stated suggests that such $p$ 's are quite rare, but should exist. We haven't found any. In particular, $K$ turns out to be $p$-rational for all $p<10,000$ and, if $p$ is a quadratic residue, even for all $p<8,000,000$, We did this verification using Sage. In the latter case, R. Pollack verified that $K$ is $p$-rational for the much larger range of primes $p<3 \times 10^{9}$. As we will explain in section 6 , if $p$ is a prime such that $4 \mid(p-1)$, and if $K$ is $p$-rational, then one can construct 3-dimensional Galois representations of $\operatorname{Gal}\left(M_{K} / \mathbf{Q}\right)$ over $\mathbf{Q}_{p}$ with open image.

\section{A Sylow pro- $p$ subgroup of $S L_{n}\left(\mathbf{Z}_{p}\right)$.}

We assume that $n \geq 2$ and that $p \geq 3$ throughout. We will first describe our notation for various groups. If $R$ is any commutative ring with identity, then $T_{n}(R)$ denotes the group of diagonal matrices in $G L_{n}(R), U_{n}(R)$ denotes the group of upper triangular matrices in $G L_{n}(R)$ with diagonal entries equal to $1_{R}$, and $B_{n}(R)=T_{n}(R) U_{n}(R)$ is the group of invertible upper triangular matrices. The $n \times n$ identity and zero matrices will be denoted by $I_{n}$ and $O_{n}$ for any ring $R$. We will also use the notation $E_{i j}$ to denote the matrix whose entries are all zeros except for a $1_{R}$ as the entry on the $i$-th row and $j$-th column. The ring, or the additive group, of $n \times n$ matrices over $R$ will be 
denoted by $M_{n}(R)$. The $R$-submodule consisting of matrices with trace equal to $0_{R}$ will be denoted by $M_{n}^{(0)}(R)$. Of course, $S L_{n}(R)$ denotes the kernel of the determinant map det $: G L_{n}(R) \rightarrow R^{\times}$.

Suppose that $R=\mathbf{Z}_{p}$. If $r \geq 1$, then the congruence subgroup $I_{n}+p^{r} M_{n}\left(\mathbf{Z}_{p}\right)$ of $G L_{n}\left(\mathbf{Z}_{p}\right)$ will be denoted by $C_{n}\left(p^{r}\right)$. Thus, $C_{n}\left(p^{r}\right)$ is a normal subgroup of $G L_{n}\left(\mathbf{Z}_{p}\right)$ and the corresponding quotient group is isomorphic to $G L_{n}\left(\mathbf{Z} / p^{r} \mathbf{Z}\right)$. The torsion subgroup of $T_{n}\left(\mathbf{Z}_{p}\right)$ will be denoted by $\Theta_{n}$. It is the subgroup of diagonal matrices whose diagonal entries are $(p-1)$-st roots of unity. For $1 \leq i \leq n$ and $\tau \in \Theta_{n}$, we let $\theta_{i}(\tau)$ denote the $i$-th diagonal entry of $\tau$. Thus, $\theta_{i}: \Theta_{n} \rightarrow \mathbf{Z}_{p}^{\times}$ is a character of $\Theta_{n}$ of order $p-1$. The reduction map $G L_{n}\left(\mathbf{Z}_{p}\right) \rightarrow G L_{n}\left(\mathbf{F}_{p}\right)$ induces a surjective homomorphism $T_{n}\left(\mathbf{Z}_{p}\right) \rightarrow T_{n}\left(\mathbf{F}_{p}\right)$. The restriction to $\Theta_{n}$ defines an isomorphism $\Theta_{n} \rightarrow T_{n}\left(\mathbf{F}_{p}\right)$. We identify those two groups and regard the characters $\theta_{i}$ as characters of $T_{n}\left(\mathbf{F}_{p}\right)$ or of $\Theta_{n}$, with values in $\mathbf{Z}_{p}^{\times}$or in $\mathbf{F}_{p}^{\times}$, depending on the context.

Let $\mathfrak{Z}=\mathbf{Z}_{p}^{n}$. We let $\left\{\mathfrak{z}_{1}, \ldots, \mathfrak{z}_{n}\right\}$ be the standard $\mathbf{Z}_{p}$-module basis for $\mathfrak{Z}$. Thus, the entries of $\mathfrak{z}_{i}$ are all 0 's, except for the $i$-th entry which is 1 . Let $G L_{n}\left(\mathbf{Z}_{p}\right)$ act on $\mathfrak{Z}$ by matrix multiplication, regarding the elements of $\mathfrak{Z}$ as column matrices. The Frattini quotient $\widetilde{\mathfrak{Z}}=\mathfrak{Z} / p \mathfrak{Z}$ is isomorphic to $\mathbf{F}_{p}^{n}$. The induced action of $G L_{n}\left(\mathbf{Z}_{p}\right)$ on $\widetilde{\mathfrak{Z}}$ factors through the quotient group $G L_{n}\left(\mathbf{F}_{p}\right)$ and is again just matrix multiplication. Since $C_{n}(p)$ is a normal pro-p-subgroup of $G L_{n}\left(\mathbf{Z}_{p}\right)$, a Sylow pro- $p$ subgroup of $G L_{n}\left(\mathbf{Z}_{p}\right)$ is determined by specifying a Sylow $p$-subgroup of $G L_{n}\left(\mathbf{F}_{p}\right)$. We can specify such a subgroup by choosing an ascending sequence of $\mathbf{F}_{p}$-subspaces $\widetilde{\mathfrak{Z}}_{i}$ for $0 \leq i \leq n$, where $\widetilde{\mathfrak{Z}}_{i}$ has dimension $i$. The set of elements of $G L_{n}\left(\mathbf{F}_{p}\right)$ which leave those subspaces fixed and which act trivially on $\widetilde{\mathfrak{Z}}_{i} / \widetilde{\mathfrak{Z}}_{i-1}$ for $i \geq 1$ is a Sylow $p$-subgroup. We simply choose $\widetilde{\mathfrak{Z}}_{i}$ to be the subspace generated by $\left\{\widetilde{\mathfrak{z}}_{1}, \ldots, \widetilde{\mathfrak{z}}_{i}\right\}$ for $i \geq 1$. The Sylow $p$-subgroup thus specified is $U_{n}\left(\mathbf{F}_{p}\right)$, as defined above. The corresponding Sylow pro-p-subgroup of $G L_{n}\left(\mathbf{Z}_{p}\right)$ will be denoted by $S_{n}\left(\mathbf{Z}_{p}\right)$. We have $S_{n}\left(\mathbf{Z}_{p}\right)=C_{n}(p) U_{n}\left(\mathbf{Z}_{p}\right)$ by definition.

5.1. Special elements of $S L_{n}\left(\mathbf{Z}_{p}\right)$. The $E_{i j}$ 's satisfy the following simple multiplication law: $E_{a b} E_{c d}=\delta_{b c} E_{a d}$, where $\delta_{b c}$ is 1 if $b=c$ and is 0 otherwise. It follows that $E_{i j}^{2}=O_{n}$ if $i \neq j$. Therefore, if $i \neq j$ and if $a$ is a positive integer, then

$$
\left(I_{n}+E_{i j}\right)^{a}=I_{n}+a E_{i j}
$$

This shows that $\left(I_{n}+E_{i j}\right)^{a} \rightarrow I_{n}$ as $a \rightarrow 0 p$-adically. Hence $\overline{\left\langle I_{n}+E_{i j}\right\rangle}$ is a pro- $p$ subgroup of $S L_{n}\left(\mathbf{Z}_{p}\right)$ and is isomorphic to $\mathbf{Z}_{p}$. Furthermore, (6) holds for all $a \in \mathbf{Z}_{p}$. If $j>i$, then $\overline{\left\langle I_{n}+E_{i j}\right\rangle} \subset U_{n}\left(\mathbf{Z}_{p}\right)$.

Suppose that $D \in T_{n}\left(\mathbf{Z}_{p}\right)$. Thus, $D=\sum_{i=1}^{n} d_{i} E_{i i}$, where $d_{i} \in \mathbf{Z}_{p}^{\times}$for $1 \leq i \leq n$. Of course, $D^{-1}=\sum_{i=1}^{n} d_{i}^{-1} E_{i i}$. The following relationship follows immediately from the multiplication law and will be quite useful:

$$
D\left(I_{n}+E_{i j}\right) D^{-1}=I_{n}+d_{i} d_{j}^{-1} E_{i j}=\left(I_{n}+E_{i j}\right)^{d_{i} d_{j}^{-1}}
$$


for any $i$ and $j$ with $i \neq j$. In particular, this applies when $D \in \Theta_{n}$ in which case we have $d_{i} d_{j}^{-1}=\left(\theta_{i} \theta_{j}^{-1}\right)(D)$. If we take $\Omega=\Theta_{n}$, then $\overline{\left\langle I_{n}+E_{i j}\right\rangle}$ is an $\Omega$-group in the sense of section 2 . More precisely, $I_{n}+E_{i j}$ is a $\chi$-element, where $\chi$ is the $\mathbf{Z}_{p}^{\times}$-valued character $\theta_{i} \theta_{j}^{-1}$ of $\Theta_{n}$

5.2. The structure of $U_{n}\left(\mathbf{F}_{p}\right)$. It is obvious that $U_{n}\left(\mathbf{F}_{p}\right)$ is a $p$-group. Every element of $U_{n}\left(\mathbf{F}_{p}\right)$ has the form $I_{n}+A$, where $A$ can be written in the form $A=\sum_{j>i} a_{i j} E_{i j}$. Here, the $a_{i j}$ 's are in $\mathbf{F}_{p}$. The following properties of $U_{n}\left(\mathbf{F}_{p}\right)$ are well-known. The Frattini subgroup $\Phi\left(U_{n}\left(\mathbf{F}_{p}\right)\right)$ consists of elements $I+A$ where $a_{i j}=0$ when $j-i=1$. Equivalently, $\Phi\left(U_{n}\left(\mathbf{F}_{p}\right)\right)$ consists of the invertible matrices which leave the subspaces $\widetilde{\mathfrak{Z}}_{i}$ fixed and which act trivially on the 2-dimensional subquotients $\widetilde{\mathfrak{Z}}_{i} / \widetilde{\mathfrak{Z}}_{i-2}$ for $2 \leq i \leq n$. It follows that

$$
U_{n}\left(\mathbf{F}_{p}\right) / \Phi\left(U_{n}\left(\mathbf{F}_{p}\right)\right) \cong \mathbf{F}_{p}^{n-1}
$$

as a group. With the above notation, the isomorphism is defined by sending $I+A$ to $\left(a_{12}, \ldots, a_{(n-1) n}\right)$. It is then clear that the set

$$
\left\{I_{n}+E_{i j} \mid j=i+1, \text { where } 1 \leq i \leq n-1\right\}
$$

is a minimal set of generators for $U_{n}\left(\mathbf{F}_{p}\right)$. Its cardinality is $n-1$.

Alternatively, one can verify that (8) generates $U_{n}\left(\mathbf{F}_{p}\right)$ by the following induction argument. It is clear for $n=2$ and, if $n \geq 3$, one can identify $U_{n-1}\left(\mathbf{F}_{p}\right)$ with the subgroup of $U_{n}\left(\mathbf{F}_{p}\right)$ consisting of elements which fix $\widetilde{\mathfrak{z}} n$. Assume that this subgroup $U_{n-1}\left(\mathbf{F}_{p}\right)$ is generated by the first $n-2$ elements in (8). Now the kernel of the restriction map $r$ defined by $r(A)=\left.A\right|_{\widetilde{\mathfrak{Z}}_{n-1}}$ is the subgroup generated by $\left\{I_{n}+E_{j n} \mid 1 \leq j \leq n-1\right\}$, which is an elementary abelian $p$-group. One sees that $U_{n}\left(\mathbf{F}_{p}\right)$ is the semidirect product of $U_{n-1}\left(\mathbf{F}_{p}\right)$ and $\operatorname{ker}(r)$. Furthermore, when $U_{n-1}\left(\mathbf{F}_{p}\right)$ acts on $\operatorname{ker}(r)$ by conjugation, one checks easily that the orbit of $I_{n}+E_{(n-1) n}$ generates $\operatorname{ker}(r)$. Thus, it would follow that (8) is a generating set for $U_{n}\left(\mathbf{F}_{p}\right)$.

5.3. The action of $U_{n}\left(\mathbf{F}_{p}\right)$ on $M_{n}\left(\mathbf{F}_{p}\right)$. We let $U_{n}\left(\mathbf{F}_{p}\right)$ act on $M_{n}\left(\mathbf{F}_{p}\right)$ by conjugation. That is, if $u \in U_{n}\left(\mathbf{F}_{p}\right)$ and $A \in M_{n}\left(\mathbf{F}_{p}\right)$, then $u$ acts by sending $A$ to $u(A)=u A u^{-1}$. Thus, $M_{n}\left(\mathbf{F}_{p}\right)$ becomes an $\mathbf{F}_{p}$-representation space for $U_{n}\left(\mathbf{F}_{p}\right)$ of degree $n^{2}$. Thus, $M_{n}\left(\mathbf{F}_{p}\right)$ can be regarded as a module over the group ring $\mathbf{F}_{p}\left[U_{n}\left(\mathbf{F}_{p}\right)\right]$. The following result is crucial for this paper.

Proposition 5.3.1. The $\mathbf{F}_{p}\left[U_{n}\left(\mathbf{F}_{p}\right)\right]$-module $M_{n}^{(0)}\left(\mathbf{F}_{p}\right)$ is cyclic. It is generated by $E_{n 1}$.

Proof. For brevity, let $U=U_{n}\left(\mathbf{F}_{p}\right)$. Consider the $\mathbf{F}_{p}$-bilinear pairing

$$
\langle\cdot, \cdot\rangle: M_{n}\left(\mathbf{F}_{p}\right) \times M_{n}\left(\mathbf{F}_{p}\right) \longrightarrow \mathbf{F}_{p}
$$

defined as follows: $\langle A, B\rangle=\operatorname{Tr}(A B)$ for all $A, B \in M_{n}\left(\mathbf{F}_{p}\right)$. It is clear that this pairing is nondegenerate. We also have $\langle u(A), u(B)\rangle=\langle A, B\rangle$ for all $u \in U$ and $A, B \in M_{n}\left(\mathbf{F}_{p}\right)$. Consequently, the $\mathbf{F}_{p}$-representation space $M_{n}\left(\mathbf{F}_{p}\right)$ for $U$ is self-dual. 
Suppose that $W$ is an $\mathbf{F}_{p}$-subspace of $M_{n}\left(\mathbf{F}_{p}\right)$. We let $W^{\perp}$ denote the orthogonal complement of $W$ with respect to the pairing $\langle\cdot, \cdot\rangle$. If $W$ is invariant under the action of $U$, then so is $W^{\perp}$. Moreover, the above pairing induces a non-degenerate $\mathbf{F}_{p}$-bilinear pairing $W^{\perp} \times\left(M_{n}\left(\mathbf{F}_{p}\right) / W\right) \rightarrow \mathbf{F}_{p}$ which is also equivariant for the action of $U$. In particular, let $W=\mathbf{F}_{p} I_{n}$. Then $W^{\perp}=M_{n}^{(0)}$ and we obtain a nondegenerate, $U$-equivariant pairing

$$
M_{n}^{(0)}\left(\mathbf{F}_{p}\right) \times\left(M_{n}\left(\mathbf{F}_{p}\right) / \mathbf{F}_{p} I_{n}\right) \longrightarrow \mathbf{F}_{p}
$$

This means that the two $\mathbf{F}_{p}$-representation spaces $M_{n}^{(0)}\left(\mathbf{F}_{p}\right)$ and $M_{n}\left(\mathbf{F}_{p}\right) / \mathbf{F}_{p} I_{n}$ for $U$ are dual to each other.

The ring $\mathbf{F}_{p}[U]$ is local. Its maximal ideal is the augmentation ideal $\mathfrak{m}$. That ideal is generated by the elements $u-I_{n}$, where $u$ varies over $U$. To prove that a nontrivial $\mathbf{F}_{p}[U]$-module $M$ is cyclic, one must show that $M / \mathfrak{m} M$ is 1-dimensional over $\mathbf{F}_{p}$. Note that $M / \mathfrak{m} M$ is the maximal quotient $M_{U}$ of $M$ on which $U$ acts trivially. If $N=\operatorname{Hom}_{\mathbf{F}_{p}}\left(M, \mathbf{F}_{p}\right)$, then $M_{U}$ is dual to $N^{U}$, the maximal submodule of $N$ on which $U$ acts trivially. Therefore, the following lemma implies proposition 5.3.1.

Lemma 5.3.2. For any $n \geq 2$ and any odd prime $p$, we have

$$
\left(M_{n}\left(\mathbf{F}_{p}\right) / \mathbf{F}_{p} I_{n}\right)^{U}=\left(\mathbf{F}_{p} I_{n}+\mathbf{F}_{p} E_{1 n}\right) / \mathbf{F}_{p} I_{n}
$$

an $\mathbf{F}_{p}$-vector space of dimension 1 .

Proof. The group $U$ acts on the $\mathbf{F}_{p}$-vector space $\widetilde{\mathfrak{Z}}$. One sees easily that the only subspaces of $\widetilde{\mathfrak{Z}}$ which are invariant under the action of $U$ are the $\widetilde{\mathfrak{Z}}_{i}$ 's, where $0 \leq i \leq n$. Note that $\left(\widetilde{\mathfrak{Z}} / \widetilde{\mathfrak{Z}}_{i}\right)^{U}=$ $\widetilde{\mathfrak{Z}}_{i+1} / \widetilde{\mathfrak{Z}}_{i}$ for $0 \leq i \leq n-1$. It will also be useful to note that the action of $U$ on the unique 2-dimensional quotient $\widetilde{\mathfrak{Z}} / \widetilde{\mathfrak{Z}}_{n-2}$ is nontrivial and that if $n \geq 3$ and $2 \leq i<n$, then $\widetilde{\mathfrak{Z}}_{i} / \widetilde{\mathfrak{Z}}_{i-2}$ is not isomorphic to $\widetilde{\mathfrak{Z}} / \widetilde{\mathfrak{Z}}_{n-2}$ as an $\mathbf{F}_{p}[U]$-module. This is clear since the action of $I_{n}+E_{(n-1) n}$ is trivial on the first module and nontrivial on the second.

We first show that $M_{n}\left(\mathbf{F}_{p}\right)^{U}=\mathbf{F}_{p} I_{n}+\mathbf{F}_{p} E_{1 n}$. The inclusion in one direction is obvious. For the other direction, suppose that $A \in M_{n}\left(\mathbf{F}_{p}\right)^{U}$. This means that $u A=A u$ for all $u \in U$. Since $\widetilde{\mathfrak{Z}}_{1}=\widetilde{\mathfrak{Z}}^{U}$, one sees that $A \widetilde{\mathfrak{Z}}_{1} \subseteq \widetilde{\mathfrak{Z}}_{1}$. A simple induction argument shows that $A \widetilde{\mathfrak{Z}}_{i} \subseteq \widetilde{\mathfrak{Z}}_{i}$ for all $i$. Thus, $A$ is upper triangular. Now $A \widetilde{\mathfrak{z}}_{1}=a \widetilde{\mathfrak{z}}_{1}$ for some $a \in \mathbf{F}_{p}$. Let $B=A-a I_{n}$, which is also in $M_{n}\left(\mathbf{F}_{p}\right)^{U}$ and is a singular matrix. It follows that $B \widetilde{\mathfrak{Z}}$ is a $U$-invariant subspace of $\widetilde{\mathfrak{Z}}$ and hence that $B \widetilde{\mathfrak{Z}}=\widetilde{\mathfrak{Z}}_{i}$ for some $i \leq n-1$. In fact, we must have $i \leq 1$. For otherwise, multiplication by $B$ would define an isomorphism from $\widetilde{\mathfrak{Z}} / \widetilde{\mathfrak{Z}}_{n-2}$ onto $\widetilde{\mathfrak{Z}}_{i} / \widetilde{\mathfrak{Z}}_{i-2}$, and this is not possible. Therefore, $B \widetilde{\mathfrak{z}}_{i}=0$ for all $i \leq n-1$ and $B \widetilde{\mathfrak{z}}_{n}=b_{\widetilde{\mathfrak{z}}_{1}}$ for some $b \in \mathbf{F}_{p}$. Consequently, $B=b E_{1 n}$. Hence, we indeed have $A=a I_{n}+b E_{1 n}$, where $a, b \in \mathbf{F}_{p}$.

Suppose that $p \nmid n$. Then $M_{n}\left(\mathbf{F}_{p}\right)$ is a direct sum $\mathbf{F}_{p} I_{n}+M_{n}^{(0)}\left(\mathbf{F}_{p}\right)$ as an $\mathbf{F}_{p}[U]$-module. We therefore have an isomorphism $M_{n}\left(\mathbf{F}_{p}\right) / \mathbf{F}_{p} I_{n} \cong M_{n}^{(0)}\left(\mathbf{F}_{p}\right)$. We also have $M_{n}^{(0)}\left(\mathbf{F}_{p}\right)^{U}=\mathbf{F}_{p} E_{1 n}$. 
Thus, the stated conclusion is now clear if $p \nmid n$. Note also that $M_{n}^{(0)}\left(\mathbf{F}_{p}\right)$ is a self-dual representation space for $U$ in this case.

If $p \mid n$, then we can use the following alternative argument which just requires that $n \geq 3$. Suppose that $A \in M_{n}\left(\mathbf{F}_{p}\right)$ and that $u A u^{-1}-A \in \mathbf{F}_{p} I_{n}$ for all $u \in U$. For any pair $(s, t)$ such that $1 \leq s<t \leq n$, let $u=I_{n}+E_{s t}$. Then $u^{-1}=I_{n}-E_{s t}$ and we have

$$
u A u^{-1}-A=E_{s t} A-A E_{s t}-E_{s t} A E_{s t},
$$

which is a matrix whose nonzero entries can only be in row $s$ or in column $t$. Since $n \geq 3$, this matrix can be equal to $c I_{n}$, where $c \in \mathbf{F}_{p}$, only if $c=0$. Thus, $u A u^{-1}=A$ for all $u$ 's of the above form. Since $U$ is generated by the set (8), it follows that $A \in M_{n}\left(\mathbf{F}_{p}\right)^{U}$. The conclusion then follows from the first step in the proof.

An element $A \in M_{n}^{(0)}\left(\mathbf{F}_{p}\right)$ will be a generator of $M_{n}^{(0)}\left(\mathbf{F}_{p}\right)$ as an $\mathbf{F}_{p}[U]$-module if and only if $A$ has a nontrivial image in $M_{n}^{(0)}\left(\mathbf{F}_{p}\right)_{U}$. This means that $A$ is not orthogonal to $E_{1 n}$. One such element is $A=E_{n 1}$. We have $\left\langle E_{n 1}, E_{1 n}\right\rangle=1$.

5.4. Generators for a Sylow pro- $p$ subgroup of $S L_{n}\left(\mathbf{Z}_{p}\right)$. We let $C_{n}^{(0)}\left(p^{r}\right)$ and $S_{n}^{(0)}\left(\mathbf{Z}_{p}\right)$ denote the intersections $C_{n}\left(p^{r}\right) \cap S L_{n}\left(\mathbf{Z}_{p}\right)$ and $S_{n}\left(\mathbf{Z}_{p}\right) \cap S L_{n}\left(\mathbf{Z}_{p}\right)$, respectively. The Sylow pro- $p$ subgroup of $S L_{n}\left(\mathbf{Z}_{p}\right)$ is $S_{n}^{(0)}\left(\mathbf{Z}_{p}\right)$, which can also be described as $C_{n}^{(0)}(p) U_{n}\left(\mathbf{Z}_{p}\right)$. Thus, $S_{n}^{(0)}\left(\mathbf{Z}_{p}\right)$ has a descending sequence of normal subgroups $C_{n}^{(0)}\left(p^{r}\right)$ for $r \geq 1, S_{n}^{(0)}\left(\mathbf{Z}_{p}\right) / C_{n}^{(0)}(p)$ is isomorphic to $U_{n}\left(\mathbf{F}_{p}\right)$, and $C_{n}^{(0)}\left(p^{r}\right) / C_{n}^{(0)}\left(p^{r+1}\right)$ is isomorphic to the additive group of $M_{n}^{(0)}\left(\mathbf{F}_{p}\right)$ for all $r \geq 1$. The latter isomorphisms are defined by the maps defined by sending the coset represented by a matrix of the form $I_{n}+p^{r} A$, where $A \in M_{n}\left(\mathbf{Z}_{p}\right)$, to the image $\widetilde{A}$ of $A$ in $M_{n}\left(\mathbf{F}_{p}\right)$. This defines an isomorphism

$$
C_{n}\left(p^{r}\right) / C_{n}\left(p^{r+1}\right) \longrightarrow M_{n}\left(\mathbf{F}_{p}\right)
$$

which is equivariant for the natural actions of $U_{n}\left(\mathbf{F}_{p}\right)$ on those two groups (defined by conjugation). This isomorphism is easily seen to send the subgroup represented by matrices of determinant 1 onto $M_{n}^{(0)}\left(\mathbf{F}_{p}\right)$. Proposition 5.3.1 then implies that $C_{n}^{(0)}\left(p^{r}\right) / C_{n}^{(0)}\left(p^{r+1}\right)$ is generated as a group by the $U_{n}\left(\mathbf{F}_{p}\right)$-orbit of the element which is represented by the matrix $I_{n}+p^{r} E_{n 1}$. Note that $I_{n}+p^{r} E_{n 1}$ is a power of $I_{n}+p E_{n 1}$ for all $r \geq 1$.

The above remarks and a straightforward induction argument give the following result.

Proposition 5.4.1. The Sylow pro-p subgroup $S_{n}^{(0)}\left(\mathbf{Z}_{p}\right)$ of $S L_{n}\left(\mathbf{Z}_{p}\right)$ can be generated topologically by

$$
\left\{I_{n}+E_{i j} \quad \mid j=i+1, \text { where } 1 \leq i \leq n-1\right\} \bigcup\left\{I_{n}+p E_{n 1}\right\}
$$

This is a minimal generating set for $S_{n}^{(0)}\left(\mathbf{Z}_{p}\right)$. It has cardinality $n$. 
Alternatively, one can verify this by using the fact that the Frattini subgroup of $C_{n}(p)$ is $C_{n}\left(p^{2}\right)$, proposition 5.3.1, and the BBT. The set (9) is a minimal topological generating set for $S_{n}^{(0)}\left(\mathbf{Z}_{p}\right)$. To see this, note that the first set in the union is contained in $U_{n}\left(\mathbf{Z}_{p}\right)$. The images of those $n-1$ elements in the Frattini quotient of $S_{n}^{(0)}\left(\mathbf{Z}_{p}\right)$ are linearly independent over $\mathbf{F}_{p}$. They don't generate $S_{n}^{(0)}\left(\mathbf{Z}_{p}\right)$ and hence cannot be a basis for the Frattini quotient of that group. Therefore, at least $n$ elements are needed to generate $S_{n}^{(0)}\left(\mathbf{Z}_{p}\right)$ topologically. We also remark that one obtains a minimal topological generating set for the Sylow pro- $p$ subgroup $S_{n}\left(\mathbf{Z}_{p}\right)$ of $G L_{n}\left(\mathbf{Z}_{p}\right)$ by just including any additional matrix $A$ such that $\operatorname{det}(A)=1+p$. For example, one can take $A=I_{n}+p E_{n n}$.

5.5. The action of $\Theta_{n}$. If we take $\Omega=\Theta_{n}$, then the elements in (9) are $\Omega$-elements. This follows from (7). The corresponding characters (in the listed order) are: $\theta_{1} \theta_{2}^{-1}, \ldots, \theta_{n-1} \theta_{n}^{-1}$, and $\theta_{n} \theta_{1}^{-1}$. These characters are all distinct. Their product is the trivial character of $\Theta_{n}$. If we choose a diagonal matrix $A$ with determinant $1+p$, then such an $A$ will also be an $\Omega$-element. The corresponding character is the trivial character of $\Theta_{n}$.

More generally, suppose that $\Omega$ is a group satisfying assumption A stated in section 2. Suppose that we fix a homomorphism $\omega: \Omega \rightarrow \Theta_{n}$. Such an $\omega$ is determined by specifying the elements $\omega_{i}=\theta_{i} \circ \omega$ of $\widehat{\Omega}$ for $1 \leq i \leq n$. We can then regard $S_{n}^{(0)}\left(\mathbf{Z}_{p}\right)$ as an $\Omega$-group. The set (9) consists of $\Omega$-elements and the corresponding characters are $\omega_{1} \omega_{2}^{-1}, \ldots, \omega_{n-1} \omega_{n}^{-1}$, and $\omega_{n} \omega_{1}^{-1}$. Of course, they are not necessarily distinct. Their product is the trivial character $\chi_{0}$ of $\Omega$. This observation determines the $\Omega$-type of $S_{n}^{(0)}\left(\mathbf{Z}_{p}\right)$. It is simply the direct sum of the above listed characters. The $\Omega$-type of $S_{n}\left(\mathbf{Z}_{p}\right)$ is then obtained by including an additional $\chi_{0}$ in the direct sum.

We now prove a result which is useful for certain applications, although we will not need it in this paper. Suppose that $(u, v)$ is a pair of integers such that $1 \leq u, v \leq n$. Let $\Omega$ and $\omega$ be as in the previous paragraph. We will say that $(u, v)$ is $(\Omega, \omega)$-distinguished if the following statement is satisfied:

$$
\text { If } 1 \leq i, j \leq n \text { and } \omega_{i} \omega_{j}^{-1}=\omega_{u} \omega_{v}^{-1} \text {, then }(i, j)=(u, v) .
$$

In particular, since $n \geq 2$, this statement implies that $u \neq v$. The following proposition will be useful in [Gr2], but only for the case where $n=2$. In that case, the pair $(1,2)$ is $(\Omega, \omega)$-distinguished if and only if the order of the character $\omega_{1} \omega_{2}^{-1}$ is not 1 or 2 .

Proposition 5.5.1. Suppose that $p-1>n$ and that $(u, v)$ is $(\Omega, \omega)$-distinguished. Suppose that $A \in G L_{n}\left(\mathbf{Z}_{p}\right)$ and that the image of $A$ in $G L_{n}\left(\mathbf{F}_{p}\right)$ is of p-power order. Suppose that $A$ is an $\Omega$-element and that the corresponding character is $\omega_{u} \omega_{v}^{-1}$. Then $A=\left(I_{n}+E_{u v}\right)^{a}$ for some $a \in \mathbf{Z}_{p}$.

Proof. First note that $A^{p^{k}} \in C_{n}(p)$ for some $k \geq 0$. Thus, $\overline{\langle A\rangle}$ is a pro- $p$ subgroup of $G L_{n}\left(\mathbf{Z}_{p}\right)$. If $r \geq 1$, then one can define a function $\log : C_{n}\left(p^{r}\right) \longrightarrow M_{n}\left(\mathbf{Z}_{p}\right)$ by the usual power series expansion. We take $r$ sufficiently large so that the image of the above map is contained in the 
domain where the power series expansion for the exponential function converges and gives a left inverse for $\log$, which we denote by exp. Then $\log$ will be injective on $C_{n}\left(p^{r}\right)$. Another property is that if $B \in C_{n}\left(p^{r}\right)$ and $b \in \mathbf{Z}_{p}$, then $\log \left(B^{b}\right)=b \cdot \log (B)$. This is a formal property of the power series defining $\log$. In addition, it is clear that if $T \in G L_{n}\left(\mathbf{Z}_{p}\right)$ and $B \in C_{n}\left(p^{r}\right)$, then $\log \left(T B T^{-1}\right)=T \log (B) T^{-1}$.

Suppose that $A$ satisfies the assumptions in the proposition. Let $\chi=\omega_{u} \omega_{v}^{-1}$, a nontrivial character of $\Omega$. Then $A^{p^{m}}$ is in $C_{n}\left(p^{r}\right)$ for some $m \geq 1$ and is a $\chi$-element. Now we can also use $\omega$ to make the additive group of $M_{n}\left(\mathbf{Z}_{p}\right)$ into an $\Omega$-group. An element $\alpha \in \Omega$ acts as conjugation by the matrix $\omega(\alpha)$. It follows that $\log \left(A^{p^{m}}\right)$ is a $\chi$-element of $M_{n}\left(\mathbf{Z}_{p}\right)$. The assumption that $(u, v)$ is $(\Omega, \omega)$-distinguished implies that $\log \left(A^{p^{m}}\right)=k E_{u v}$ for some $k \in \mathbf{Z}_{p}$. Since $u \neq v$, we have $E_{u v}^{2}=O_{n}$. As a consequence of these remarks, together with (6), we have

$$
A^{p^{m}}=\exp \left(k E_{u v}\right)=I_{n}+k E_{u v}=\left(I_{n}+E_{u v}\right)^{k}
$$

for some $k \in \mathbf{Z}_{p}$. In particular, $A^{p^{m}}$ is a unipotent matrix. Thus, the eigenvalues of $A$ are $p$-power roots of unity. Since $n<p-1=\left[\mathbf{Q}_{p}\left(\mu_{p}\right): \mathbf{Q}_{p}\right]$, it follows that $A$ itself is unipotent.

If $k=0$, then one sees easily that $A=I_{n}$ and one can take $a=0$. We now assume that $k \neq 0$. Consider $A$ and $E_{u v}$ as endomorphisms of the vector space $\mathcal{V}=\mathfrak{Z} \otimes \mathbf{z}_{p} \mathbf{Q}_{p}$. Note that $A^{p^{m}}-I_{n}=\left(A-I_{n}\right) B$, where $B$ is invertible in $M_{n}\left(\mathbf{Q}_{p}\right)$. Thus, $A-I_{n}$ and $A^{p^{m}}-I_{n}$ have the same kernel and the same image as endomorphisms of $\mathcal{V}$, which are also the same as the kernel and the image of $E_{u v}$, respectively. The common kernel has codimension 1 and is generated by the $\mathfrak{z} i$ 's with $i \neq t$. The common image is $\mathbf{Q}_{p} \mathfrak{z}_{u}$ and so $\left(A-I_{n}\right) \mathfrak{z}_{v}=a \mathfrak{z}_{u}$ for some $a \in \mathbf{Q}_{p}$. Clearly, $a \in \mathbf{Z}_{p}$. It follows that $A-I_{n}=a E_{u v}$ and the stated conclusion then follows by using (6).

5.6. Automorphisms of $S_{n}^{(0)}\left(\mathbf{Z}_{p}\right)$. Let $s_{1}, \ldots, s_{n}$ be the topological generators for $S_{n}^{(0)}\left(\mathbf{Z}_{p}\right)$ listed in (9). A continuous automorphism $\delta$ of $S_{n}^{(0)}\left(\mathbf{Z}_{p}\right)$ is determined if one specifies $\delta\left(s_{i}\right)$ for $1 \leq i \leq n$. If we take $\Omega=\Theta_{n}$ and $\omega$ to be the identity map, then every pair $(u, v)$ with $u \neq v$ is $(\Omega, \omega)$ distinguished. Assuming that $p-1>n$, it then follows from proposition 5.5.1 that any continuous $\Omega$-automorphism $\delta$ of $S_{n}^{(0)}\left(\mathbf{Z}_{p}\right)$ must have the property that $\delta\left(s_{i}\right)=s_{i}^{a_{i}}$ where $a_{i} \in \mathbf{Z}_{p}$. Thus, $\delta$ is determined by the $n$-tuple $\left(a_{1}, \ldots, a_{n}\right)$. The following result shows that $\delta$ is just conjugation by a certain matrix in $T_{n}\left(\mathbf{Z}_{p}\right)$. The constraint on the product of the $a_{i}$ 's will be useful in the proof of proposition 6.1.2. No assumption about $p$ is needed.

Proposition 5.6.1. Suppose that $a_{1}, \ldots, a_{n} \in \mathbf{Z}_{p}$. There exists a continuous automorphism $\delta$ of $S_{n}^{(0)}\left(\mathbf{Z}_{p}\right)$ such that $\delta\left(s_{i}\right)=s_{i}^{a_{i}}$ for $1 \leq i \leq n$ if and only if $\prod_{i=1}^{n} a_{i}=1$. For any such automorphism $\delta$, there exists a matrix $t \in T_{n}\left(\mathbf{Z}_{p}\right)$ such that $\delta(s)=t s t^{-1}$ for all $s \in S_{n}^{(0)}\left(\mathbf{Z}_{p}\right)$.

Proof. Suppose first that $a_{1}, \ldots, a_{n} \in \mathbf{Z}_{p}$ and that $\prod_{i=1}^{n} a_{i}=1$. We can then define $t_{1}, \ldots, t_{n} \in \mathbf{Z}_{p}^{\times}$ such that

$$
a_{1}=t_{1} t_{2}^{-1}, \ldots ., a_{n-1}=t_{n-1} t_{n}^{-1}, \quad a_{n}=t_{n} t_{1}^{-1} .
$$


Let $t=\sum_{i=1}^{n} t_{i} E_{i i}$. We then have $\delta\left(s_{i}\right)=s_{i}^{a_{i}}$ for $1 \leq i \leq n$. This follows from (7). Obviously, $\delta$ is a continuous automorphism of $S_{n}^{(0)}\left(\mathbf{Z}_{p}\right)$.

For the converse, by composing a given $\delta$ with the automorphism of $S_{n}^{(0)}\left(\mathbf{Z}_{p}\right)$ defined by conjugation by a suitable diagonal matrix $t$, we can reduces to the case where $a_{1}, \ldots, a_{n-1}$ are all 1 's. Letting $a=a_{n}$, it then suffices to show that $a=1$. Thus, we can now assume that $\delta$ fixes $s_{1}, \ldots, s_{n-1}$ and that $\delta\left(s_{n}\right)=s_{n}^{a}$. Recall that $\left\{s_{1}, \ldots, s_{n-1}\right\}$ is a topological generating set for $U_{n}\left(\mathbf{Z}_{p}\right)$. It follows that $\delta$ fixes the elements of $U_{n}\left(\mathbf{Z}_{p}\right)$. Proposition 5.3.1 implies that $C_{n}^{(0)}(p)$ has a topological generating set consisting of conjugates of $s_{n}=I_{n}+p E_{n 1}$ by elements of $U_{n}\left(\mathbf{Z}_{p}\right)$. This follows from the facts that the Frattini subgroup of $C_{n}^{(0)}(p)$ is $C_{n}^{(0)}\left(p^{2}\right)$ and that there is a $U_{n}\left(\mathbf{F}_{p}\right)$-equivariant isomorphism of $C_{n}^{(0)}(p) / C_{n}^{(0)}\left(p^{2}\right)$ to the additive group $M_{n}^{(0)}\left(\mathbf{F}_{p}\right)$. It is clear that if $u \in U_{n}\left(\mathbf{Z}_{p}\right)$ and if $c=u s_{n} u^{-1}$, then $\delta(c)=c^{a}$. Therefore, $C_{n}^{(0)}(p)$ has a topological generating set consisting of elements $c_{j}$, where $1 \leq j \leq n^{2}-1$, such that $\delta\left(c_{j}\right)=c_{j}^{a}$ for all $j$ 's.

We will use theorems 4.3.1, 5.3.2, and corollary 9.23 from [DSMS]. They imply that $C_{n}^{(0)}(p)$ is a $p$-adic analytic group and that its elements can be uniquely expressed in the form

$$
\prod_{j=1}^{n^{2}-1} c_{j}^{x_{j}}
$$

where the $x_{j}$ 's are in $\mathbf{Z}_{p}$ and can be taken as the coordinates defining the analytic structure on $C_{n}^{(0)}(p)$. The effect of applying $\delta$ to such an element is to multiply the corresponding coordinates by $a$. Hence $\delta$ is an analytic automorphism of $C_{n}^{(0)}(p)$. We then get an automorphism $\mathfrak{d}$ of the Lie algebra $\mathfrak{s l}_{n}$ for $C_{n}^{(0)}(p)$, a Lie algebra over $\mathbf{Q}_{p}$. For each $j$, the subgroup $\overline{\left\langle c_{j}\right\rangle}$ of $C_{n}^{(0)}(p)$ is a 1dimensional analytic subgroup on which $\delta$ acts by the map $c \rightarrow c^{a}$. Thus $\mathfrak{d}$ acts on the corresponding 1-dimensional subalgebra of $\mathfrak{s l}_{n}$ as multiplication by $a$. Since $\mathfrak{d}$ is $\mathbf{Q}_{p}$-linear and those subalgebras generate $\mathfrak{s l}_{n}$ as a $\mathbf{Q}_{p}$-vector space, it follows that $\mathfrak{d}$ acts as multiplication by $a$ on $\mathfrak{s l}_{n}$ and therefore on the Lie subalgebra $\mathfrak{u}_{n}$ corresponding to $U_{n}\left(\mathbf{Z}_{p}\right)$. Since $\delta$ acts trivially on all of $U_{n}\left(\mathbf{Z}_{p}\right)$, $\mathfrak{d}$ will act trivially on $\mathfrak{u}_{n}$. It follows that $a=1$.

5.7. The $\Omega$-type of the pro- $p$ group $C_{n}(p)$. We assume that $\Omega$ has order prime to $p$ and that we are given a homomorphism $\bar{\omega}: \Omega \rightarrow G L_{n}\left(\mathbf{F}_{p}\right)$. Then we know that $\bar{\omega}$ can be lifted to a homomorphism $\omega: \Omega \rightarrow G L_{n}\left(\mathbf{Z}_{p}\right)$. If $\alpha \in \Omega$, then we can let $\alpha$ act on $C_{n}(p)$ as conjugation by the matrix $\omega(\alpha)$. Thus, $C_{n}(p)$ becomes an $\Omega$-group. The Frattini quotient is

$$
\widetilde{C_{n}(p)}=C_{n}(p) / C_{n}\left(p^{2}\right) \cong M_{n}\left(\mathbf{F}_{p}\right) \text {. }
$$

Thus, the minimal cardinality of a topological generating set for $C_{n}(p)$ is $n^{2}$. The $\Omega$-type of $C_{n}(p)$ is determined by $\bar{\omega}$ and is defined by letting $\alpha \in \Omega$ act on $M_{n}\left(\mathbf{F}_{p}\right)$ as conjugation by the matrix $\bar{\omega}(\alpha)$. This representation of $\Omega$ is isomorphic to the tensor product (over $\mathbf{F}_{p}$ ) of $\bar{\omega}$ and the contragredient of 
$\bar{\omega}$. If we let $\check{\omega}$ denote the contragredient of $\omega$, then the $\Omega$-type of $C_{n}(p)$ is obtained from $\omega \otimes \mathbf{z}_{p} \check{\omega}$ by reduction modulo $p$. The resulting representation of $\Omega$ over $\mathbf{F}_{p}$ is the so-called adjoint represention (corresponding to $\bar{\omega}$ ) and will be denoted by $\operatorname{ad}(\bar{\omega})$.

\section{The construction.}

We assume that $K / \mathbf{Q}$ is a finite abelian extension, that $p$ is an odd prime, and that $\Omega=\operatorname{Gal}(K / \mathbf{Q})$ has exponent dividing $p-1$. As in section 3 , we let $M=M_{K}$ denote the maximal pro- $p$ extension of $K$ which is unramified outside of the set of primes above $p$. Let $\Gamma=\Gamma_{K}=\operatorname{Gal}(M / K)$. The action of $G_{\mathbf{Q}}$ on $\mu_{p^{\infty}}$ defines a continuous homomorphism $\chi_{c y c}$ from $G_{\mathbf{Q}}$ to $G L_{1}\left(\mathbf{Z}_{p}\right)=\mathbf{Z}_{p}^{\times}$. It is surjective. Now $1+p \mathbf{Z}_{p}$ is a direct factor in $\mathbf{Z}_{p}^{\times}$. Composing $\chi_{c y c}$ with the projection map defines a surjective homomorphism $\kappa: G_{\mathbf{Q}} \rightarrow 1+p \mathbf{Z}_{p}$. One sees easily that $\kappa$ factors through $\operatorname{Gal}(M / \mathbf{Q})$.

6.1. The basic proposition. Our construction of continuous representations into $G L_{n}\left(\mathbf{Z}_{p}\right)$ with open image is based on the following result.

Proposition 6.1.1. Assume that $K$ is p-rational and that $\Omega=\operatorname{Gal}(K / \mathbf{Q})$ has exponent dividing $p-1$. Assume also that one can find distinct characters $\chi_{1}, \ldots, \chi_{n}$ in $\widehat{\Omega}_{\text {odd }} \cup\left\{\chi_{0}\right\}$ such that their product is $\chi_{0}$. Then there exists a continuous homomorphism

$$
\rho_{0}: \operatorname{Gal}(M / \mathbf{Q}) \longrightarrow G L_{n}\left(\mathbf{Z}_{p}\right)
$$

such that $\rho_{0}(\Gamma)=S_{n}^{(0)}\left(\mathbf{Z}_{p}\right)$. Furthermore, $\rho=\rho_{0} \otimes \kappa$ is a continuous homomorphism from $\operatorname{Gal}(M / \mathbf{Q})$ to $G L_{n}\left(\mathbf{Z}_{p}\right)$ with open image.

Proof. We may as well assume that $n \geq 2$. The result is trivial for $n=1$. Thus, $\widehat{\Omega}_{\text {odd }}$ is nonempty and $K$ is totally complex. If $\omega: \Omega \rightarrow \Theta_{n}$ is a homomorphism, then we let $\omega_{i}=\theta_{i} \circ \omega$ for $1 \leq i \leq n$. The $\omega_{i}$ 's are in $\widehat{\Omega}$. We specify $\omega$ by choosing the $\omega_{i}$ 's so that

$$
\omega_{1} \omega_{2}^{-1}=\chi_{1}, \quad . \quad . \quad . \quad, \quad \omega_{n-1} \omega_{n}^{-1}=\chi_{n-1}, \quad \text { and } \quad \omega_{n} \omega_{1}^{-1}=\chi_{n} .
$$

If we choose $\omega_{1} \in \widehat{\Omega}$ arbitrarily, and choose $\chi_{1}, \ldots, \chi_{n}$ as stated in the proposition, then the first $n-1$ of these equations will determine a certain $\omega$. The assumption about the product of the $\chi_{i}$ 's makes the $n$-th equation satisfied too.

Note that $\Theta_{n}$ normalizes $S_{n}^{(0)}\left(\mathbf{Z}_{p}\right)$. If $\alpha \in \Omega$, then we can let $\alpha$ act on $S_{n}^{(0)}\left(\mathbf{Z}_{p}\right)$ as conjugation by $\omega(\alpha)$. Hence, $S_{n}^{(0)}\left(\mathbf{Z}_{p}\right)$ becomes an $\Omega$-group. Furthermore, there is a homomorphism from the corresponding semidirect product $S_{n}^{(0)}\left(\mathbf{Z}_{p}\right) \rtimes \Omega$ to $G L_{n}\left(\mathbf{Z}_{p}\right)$. It is defined by making it the identity map on $S_{n}^{(0)}\left(\mathbf{Z}_{p}\right)$ and the map $\omega$ on $\Omega$. 
As pointed out in section 5.5, the generators of $S_{n}^{(0)}\left(\mathbf{Z}_{p}\right)$ listed in proposition 5.4.1 are $\Omega$ elements and the corresponding characters are $\chi_{1}, \ldots, \chi_{n}$, respectively. We will denote them by $s_{1}, \ldots, s_{n}$ in order. Thus, it is clear that the $\Omega$-type of $S_{n}^{(0)}\left(\mathbf{Z}_{p}\right)$ is bounded above by the $\Omega$-type of the free pro- $p$ group $\Gamma=\Gamma_{K}$ which is described in corollary 3.2.2. Recall from section 3.2 that $\operatorname{Gal}(M / \mathbf{Q})$ is isomorphic to a semidirect product $\Gamma \rtimes \Omega$. This is how one makes $\Gamma$ into an $\Omega$ group. By proposition 2.1.1, we can choose a topological generating set $\gamma_{1}, \ldots, \gamma_{r}$ for $\Gamma$ consisting of $\Omega$ elements, where $\frac{1}{2}[K: \mathbf{Q}]+1$. We have $n \leq r$. Choose the indexing so that $\gamma_{i}$ is a $\chi_{i}$-element for $1 \leq i \leq n$.

We can define a surjective homomorphism $\sigma_{0}: \Gamma \rightarrow S_{n}^{(0)}\left(\mathbf{Z}_{p}\right)$ by mapping $\gamma_{i}$ to $s_{i}$ for $1 \leq i \leq n$ and mapping the $\gamma_{i}$ 's for $i>n$ (if there are any) to $I_{n}$. It is clear that $\sigma_{0}$ is an $\Omega$-homomorphism. Therefore, we can extend $\sigma_{0}$ to a surjective homomorphism from $\Gamma \rtimes \Omega$ to $S_{n}^{(0)}\left(\mathbf{Z}_{p}\right) \rtimes \Omega$. This then gives us a homomorphism from $\rho_{0}$ from $\operatorname{Gal}(M / \mathbf{Q})$ to $G L_{n}\left(\mathbf{Z}_{p}\right)$ whose image contains $S_{n}^{(0)}\left(\mathbf{Z}_{p}\right)$. smallskip

Consider the representation $\rho=\rho_{0} \otimes \kappa$ of $\operatorname{Gal}(M / \mathbf{Q})$. Denote $M^{\operatorname{ker}\left(\rho_{0}\right)}$ by $\mathbf{Q}\left(\rho_{0}\right)$. Thus, $\operatorname{Gal}\left(\mathbf{Q}\left(\rho_{0}\right) / \mathbf{Q}\right)$ is isomorphic to the image of $\rho_{0}$, a $p$-adic Lie group whose Lie algebra is $\mathfrak{s l}_{n}$. It follows that the maximal abelian quotient of $\operatorname{Gal}\left(\mathbf{Q}\left(\rho_{0}\right) / \mathbf{Q}\right)$ is finite. The field $M^{\mathrm{ker}(\kappa)}$ is just the cyclotomic $\mathbf{Z}_{p}$-extension $\mathbf{Q}_{\infty}$ of $\mathbf{Q}$. Since $\mathbf{Q}_{\infty}$ is an abelian extension of $\mathbf{Q}$, it follows that $\mathbf{Q}\left(\rho_{0}\right) \cap \mathbf{Q}_{\infty}$ is a finite extension of $\mathbf{Q}$. Let $F=\mathbf{Q}\left(\rho_{0}\right) \mathbf{Q}_{\infty}$. Both $\rho_{0}$ and $\kappa$ can be regarded as representations of $\operatorname{Gal}(F / \mathbf{Q})$. The restrictions of $\rho$ and $\rho_{0}$ to $\operatorname{Gal}\left(F / \mathbf{Q}_{\infty}\right)$ coincide and their image contains an open subgroup of $S L_{n}\left(\mathbf{Z}_{p}\right)$. On the other hand, the restriction of $\rho$ to $\operatorname{Gal}\left(F / \mathbf{Q}\left(\rho_{0}\right)\right)$ coincides with the restriction of $\kappa$, viewed as having its values in the group $\mathbf{Z}_{p}^{\times} I_{n}$ of scalar matrices in $G L_{n}\left(\mathbf{Z}_{p}\right)$. The image will be an open subgroup of $\mathbf{Z}_{p}^{\times} I_{n}$. Thus, the image of $\rho$ contains open subgroups of both $S L_{n}\left(\mathbf{Z}_{p}\right)$ and $\mathbf{Z}_{p}^{\times} I_{n}$, and hence must indeed be an open subgroup of $G L_{n}\left(\mathbf{Z}_{p}\right)$.

Proposition 6.1.2. Under the assumptions of proposition 6.1.1, there exists an uncountable collection of homomorphisms $\rho_{0}$ with the stated properties and such that the corresponding kernels are distinct.

Proof. Assume that $K, p$, and $n$ satisfy the assumptions in proposition 6.1.1. Fix a choice of $\chi_{1}, \ldots, \chi_{n}$ and define $\omega: \Omega \rightarrow \Theta_{n}$ as in the proof. Thus, $S_{n}^{(0)}\left(\mathbf{Z}_{p}\right)$ can be regarded as an $\Omega$-group. One obtains a $\rho_{0}$ for each choice of a surjective $\Omega$-homomorphism $\sigma_{0}$ from $\Gamma$ to $S_{n}^{(0)}\left(\mathbf{Z}_{p}\right)$. Fix a topological generating set $\gamma_{1}, \ldots, \gamma_{r}$ for $\Gamma$ as in the proof of proposition 6.1.1. We continue to let $s_{1}, \ldots, s_{n}$ be the topological generators for $S_{n}^{(0)}\left(\mathbf{Z}_{p}\right)$ given in (9). We will assume that $\sigma_{0}$ is defined by mapping $\gamma_{i}$ to $s_{i}$ for $1 \leq i \leq n$ and by mapping the remaining generators $\gamma_{i}$ for $i>n$ (if $r$ exceeds $n$ ) to the identity element $I_{n}$ of $S_{n}^{(0)}\left(\mathbf{Z}_{p}\right)$.

If $\psi$ is any $\Omega$-automorphism of $\Gamma_{K}$, then $\sigma \circ \psi$ will be another surjective $\Omega$-homomorphism from $\Gamma_{K}$ to $S_{n}^{(0)}\left(\mathbf{Z}_{p}\right)$. We have

$$
\operatorname{ker}(\sigma \circ \psi)=\psi^{-1}(\operatorname{ker}(\sigma))
$$


For simplicity, we will restrict attention to automorphisms $\psi$ of the following special form:

$$
\psi\left(\gamma_{i}\right)=\gamma_{i}^{a_{i}} \quad \text { for } 1 \leq i \leq n, \quad \psi\left(\gamma_{i}\right)=\gamma_{i} \quad \text { for } i>n
$$

where the $a_{i}$ 's are in $\mathbf{Z}_{p}^{\times}$. Any such automorphism is clearly an $\Omega$-automorphism. We refer to $a_{1}, \ldots, a_{n}$ as the parameters for $\psi$. Since $\Gamma_{K}$ is free, any choice of $a_{1}, \ldots, a_{n}$ will determine such an automorphism $\psi$ of $\Gamma_{K}$. Note that $\sigma \circ \psi$ also maps the $\gamma_{i}$ 's for $i>n$ to $I_{n}$.

Suppose that $\psi^{\prime}$ is another automorphism of $\Gamma$ of the form (10) and that the corresponding parameters are $a_{1}^{\prime}, \ldots, a_{n}^{\prime}$. We will prove that

$$
\operatorname{ker}(\sigma \circ \psi)=\operatorname{ker}\left(\sigma \circ \psi^{\prime}\right) \Longleftrightarrow \prod_{i=1}^{n} a_{i}=\prod_{i=1}^{n} a_{i}^{\prime}
$$

and hence that there are indeed uncountably many distinct kernels for the homomorphisms $\sigma \circ \psi$. In effect, we are just replacing one set of topological generators for $\Gamma_{K}$ by other choices, each consisting of $\Omega$-elements. We thereby get many $\rho_{0}$ 's and uncountably many distinct kernels.

One easily reduces to the case where $\psi^{\prime}$ is the identity automorphism of $\Gamma_{K}$, i.e., where $a_{i}^{\prime}=1$ for $1 \leq i \leq n$. Thus, we must show that $\sigma \circ \psi$ and $\sigma$ have the same kernel if and only if $\prod_{i=1}^{n} a_{i}=1$. This assertion follows from proposition 5.6.1. To see this, first assume that $\sigma \circ \psi$ and $\sigma$ have the same kernel. Thus, $\operatorname{ker}(\sigma)$ is fixed by $\psi$. It follows that $\psi$ will induce an automorphism on the quotient group $\Gamma_{K} / \operatorname{ker}(\sigma)$, and hence on the group $S_{n}^{(0)}\left(\mathbf{Z}_{p}\right)$. Calling that automorphism $\delta$, we have

$$
\sigma \circ \psi=\delta \circ \sigma
$$

Conversely, the existence of such a $\delta$ implies that $\sigma \circ \psi$ and $\sigma$ have the same kernel. Note that (11) implies that $\delta\left(s_{i}\right)=s_{i}^{a_{i}}$ for $1 \leq i \leq n$, where $a_{1}, \ldots, a_{n}$ are the parameters for $\psi$. According to proposition 5.6.1, such a $\delta$ exists if and only if $\prod_{i=1}^{n} a_{i}=1$.

Remark 6.1.3. Following the notation in the proof of proposition 6.1.2, suppose that $\sigma$ and $\sigma^{\prime}$ are two surjective homomorphisms from $\Gamma_{K}$ to $S_{n}^{(0)}\left(\mathbf{Z}_{p}\right)$ and that $\operatorname{ker}(\sigma) \neq \operatorname{ker}\left(\sigma^{\prime}\right)$. Then $\operatorname{ker}(\sigma) \operatorname{ker}\left(\sigma^{\prime}\right)$ is a normal subgroup of $\Gamma_{K}$ and the corresponding quotient group is isomorphic to a proper quotient group of $S_{n}^{(0)}\left(\mathbf{Z}_{p}\right)$. The Lie algebra of $S_{n}^{(0)}\left(\mathbf{Z}_{p}\right)$ is $\mathfrak{s l}_{n}$, a simple Lie algebra over $\mathbf{Q}_{p}$. One shows easily that $S_{n}^{(0)}\left(\mathbf{Z}_{p}\right)$ has no nontrivial finite, normal subgroups. Thus, a closed normal subgroup of $S_{n}^{(0)}\left(\mathbf{Z}_{p}\right)$ must have finite index. In particular, $\operatorname{ker}(\sigma) \operatorname{ker}\left(\sigma^{\prime}\right)$ has finite index in $\Gamma_{K}$. Therefore, the intersection of the fields cut out by the representations $\sigma$ and $\sigma^{\prime}$ must be a finite extension of $K$. If one constructs representations $\rho$ and $\rho^{\prime}$ of $G_{\mathbf{Q}}$ from $\sigma$ and $\sigma^{\prime}$ as in proposition 6.1.1, so that their images are open, then the intersection of the fields cut out by $\rho$ and $\rho^{\prime}$ will be a finite extension of $\mathbf{Q}_{\infty}$

Remark 6.1.4. It is natural to ask whether the uncountable family of representations $\rho$ constructed in the proof of proposition 6.1.1 includes some representations having nice arithmetic properties 
at $p$. Could one make the construction so that the restriction of $\rho$ to a decomposition subgroup is crystalline or Hodge-Tate, for example? We don't see how to deal with such questions. The difficulties become clear by considering two extreme cases.

Suppose first that there is just one prime $\mathfrak{p}$ of $K$ lying over $p$, where $K$ satisfies the assumptions in proposition 6.1.1. Suppose also that $p$ doesn't divide the class number of $K$. Then one sees easily that $\mathfrak{p}$ is totally ramified in $M / K$ and therefore that $\Gamma_{K}$ can be identified with $\operatorname{Gal}\left(M_{\mathfrak{p}} / K_{\mathfrak{p}}\right)$, where $M_{\mathfrak{p}}$ is a certain pro- $p$ extension of $K_{\mathfrak{p}}$. However, we don't see any way to identify that extension. There may indeed be $n$-dimensional representations of the local Galois group $G_{K_{\mathfrak{p}}}$ with open image which have some nice properties (a question which we haven't examined), but how can one construct such representations so that they factor through the quotient group $\operatorname{Gal}\left(M_{\mathfrak{p}} / K_{\mathfrak{p}}\right)$.

Suppose now that $p$ splits completely in $K / \mathbf{Q}$. Let $\mathfrak{p}$ be one of the primes of $K$ lying over $p$. Thus, $K_{\mathfrak{p}}=\mathbf{Q}_{p}$. If $K$ is a complex, abelian extension of $\mathbf{Q}$, then one can show that the decomposition subgroup of $\Gamma_{K}^{a b}$ for $\mathfrak{p}$ is isomorphic to $\mathbf{Z}_{p}^{2}$. This is a nontrivial fact. It follows as a consequence of proposition 3 in [Gr73]. Thus, the decomposition subgroup $D_{\mathfrak{p}}$ of $\Gamma_{K}$ for a prime of $M$ lying above $\mathfrak{p}$ requires at least two topological generators. One can identify $D_{\mathfrak{p}}$ with $\operatorname{Gal}\left(M_{\mathfrak{p}} / \mathbf{Q}_{p}\right)$, where $M_{\mathfrak{p}}$ is a certain pro- $p$ extension of $\mathbf{Q}_{p}$. Furthermore, if one assumes that $K$ is $p$-rational, then $D_{\mathfrak{p}}$ must be a free pro- $p$ group since it is a subgroup of the free pro- $p$ group $\Gamma_{K}$.

Now one can show that the Galois group $\Gamma_{\mathbf{Q}_{p}}$ for the maximal pro- $p$ extension of $\mathbf{Q}_{p}$ is a free pro- $p$ group on 2 generators. Since there is a surjective homomorphism from $\Gamma_{\mathbf{Q}_{p}}$ to $D_{\mathfrak{p}}$, the facts mentioned above imply that such a homomorphism is injective and hence that $M_{\mathfrak{p}}$ is precisely the maximal pro- $p$ extension of $\mathbf{Q}_{p}$. The difficulty is that we cannot give a description of the subgroup $D_{\mathfrak{p}}$ in terms of the type of generating set for $\Gamma_{K}$ described in proposition 3.2.1. Thus, it is not clear how to study the restrictions $\left.\rho\right|_{D_{\mathfrak{p}}}$ for the representations $\rho$ constructed in propositions 6.1.1.

Remark 6.1.5. The image of the representation $\rho$ in proposition 6.1 .1 contains $S_{n}\left(\mathbf{Z}_{p}\right)$ if $n$ is even and $p \nmid n$. To see this, note that if $n$ is even, then the characters $\chi_{1}, \ldots, \chi_{n}$ will all be odd. Referring to the proof of the proposition, one sees that the $\Omega$-type of $\sigma_{0}(\Gamma)=S_{n}^{(0)}\left(\mathbf{Z}_{p}\right)$ doesn't contain $\chi_{0}$. Let $K\left(\sigma_{0}\right)=M^{\operatorname{ker}\left(\sigma_{0}\right)}$ and $K_{\infty}=K \mathbf{Q}_{\infty}$, which is the fixed field for $\left.\kappa\right|_{\Gamma}$. We then have

$$
K\left(\sigma_{0}\right) \cap K_{\infty}=K
$$

Therefore, the image of $\operatorname{Gal}\left(M / K_{\infty}\right)$ under $\sigma_{0}$, and hence under $\rho$, will still be $S_{n}^{(0)}\left(\mathbf{Z}_{p}\right)$. Thus, the image of $\rho$ contains $S_{n}^{(0)}\left(\mathbf{Z}_{p}\right)$. It then suffices to show that the image of $\operatorname{det}(\rho)$ contains $1+p \mathbf{Z}_{p}$. However, $\kappa(\Gamma)=\kappa\left(\operatorname{ker}\left(\sigma_{0}\right)\right)$ is $1+p \mathbf{Z}_{p}$. The restriction of $\rho$ to $\operatorname{ker}\left(\sigma_{0}\right)$ is just a direct sum of $n$ copies of $\kappa$. The image under the determinant map is $1+p \mathbf{Z}_{p}$ since $p \nmid n$.

6.2. Finding suitable character sets. Now we discuss the existence of a set of characters $\chi_{1}, \ldots, \chi_{n}$ with the properties stated in the above proposition. Suppose first that $K$ is a cyclic extension of $\mathbf{Q}$ and that $K$ is complex. Then $[K: \mathbf{Q}]=2 g$ for some $g$. We have $\left|\widehat{\Omega}_{\text {odd }}\right|=g$. Assume that $g \geq 2$. Consider pairs of odd characters $\left\{\chi, \chi^{-1}\right\}$, where $\chi^{-1} \neq \chi$. The number of such pairs 
is $[g / 2]$. Thus, if $n$ is even and $n \leq 2[\mathrm{~g} / 2]$, then we can take $n / 2$ such pairs to obtain $n$ distinct, odd characters whose product is $\chi_{0}$. If $n$ is odd, then we can choose one of the characters to be $\chi_{0}$ and choose the other characters in pairs as above. We then obtain a suitable set of $n$ characters if $n-1 \leq 2[g / 2]$. In both cases, the inequality we need for $n$ can be stated as $[n / 2] \leq g / 2$. Thus, we obtain the following result.

Proposition 6.2.1. Suppose that $n \geq 2$. Suppose that $K$ is a complex, cyclic extension of $\mathbf{Q}$, that $[K: \mathbf{Q}]$ divides $p-1$, that $K$ is p-rational, and that $[K: \mathbf{Q}] \geq 4[n / 2]$. Then there exist continuous homomorphisms $\rho_{0}$ and $\rho$ having the properties stated in proposition 6.1.1.

Proposition 1.1 in the introduction is the special case $K=\mathbf{Q}\left(\mu_{p}\right)$. The assumption there is that $p$ is a regular prime. Hence $K$ is $p$-rational by proposition 4.3.1. However, even if $p$ is irregular, the construction sometimes works. As an example, suppose that $p=37$. Then the torsion subgroup of $\left(\Gamma_{K}^{a b}\right)^{e_{\chi}}$ is nontrivial for exactly one $\chi$, namely $\chi=\varepsilon^{32}$ where $\varepsilon$ denotes the character giving the action of $\operatorname{Gal}(K / \mathbf{Q})$ on $\mu_{p}$. It follows from the discussion in remark 3.2.4 that the unique subfield $K^{\prime}$ of $K$ such that $\left[K^{\prime}: \mathbf{Q}\right]=12$ is $p$-rational. Thus, one can apply proposition 6.1 .3 for $p=37$ to the field $K^{\prime}$ provided that $2 \leq n \leq 7$.

Another special case is $K=\mathbf{Q}\left(\mu_{5}\right)$. Since $[K: \mathbf{Q}]=4$, we assume that $p \equiv 1(\bmod 4)$. Except for the seemingly rare primes $p$ for which $K$ fails to be $p$-rational, one can then apply proposition 6.1.3, taking $n$ to be 2 or 3 . In particular, one obtains representations $\rho: G_{\mathbf{Q}} \rightarrow G L_{3}\left(\mathbf{Z}_{p}\right)$ for an extremely large set of primes, including all primes $p<10,000$ such that $p \equiv 13$ or $17(\bmod 20)$ and all primes $p<3 \times 10^{9}$ such that $p \equiv 1$ or $9(\bmod 20)$.

Now consider the case where $K$ is a compositum of quadratic fields. Thus, $\Omega$ is an elementary abelian 2-group. The exponent of $\Omega$ certainly divides $p-1$. We prove the following result.

Proposition 6.2.2. Suppose that $K$ is complex and that $\Omega=\operatorname{Gal}(K / \mathbf{Q})$ is isomorphic to $(\mathbf{Z} / 2 \mathbf{Z})^{t}$, where $t \geq 4$. Suppose also that $4 \leq n \leq 2^{t-1}-3$. Then one can find distinct characters $\chi_{1}, \ldots, \chi_{n}$ in $\widehat{\Omega}_{\text {odd }} \cup\left\{\chi_{0}\right\}$ whose product is $\chi_{0}$. Consequently, if we also assume that $K$ is p-rational, then there exists continuous homomorphisms $\rho_{0}$ and $\rho$ having the properties stated in proposition 6.1.1.

Proof. Let $\widehat{\Omega}_{e v}$ denote the subgroup of $\widehat{\Omega}$ consisting of even characters, which has order $2^{t-1}$. Let $m=2[n / 2]$. Then $m$ is even and $4 \leq m \leq n$. We will show that there exists distinct elements $\varphi_{1}, \ldots, \varphi_{m} \in \widehat{\Omega}_{e v}$ whose product is $\chi_{0}$. If $\psi$ is any element of $\widehat{\Omega}_{o d d}$, then one can take $\chi_{i}=\psi \varphi_{i}$ for $1 \leq i \leq m$. These $\chi_{i}$ 's are distinct elements of $\widehat{\Omega}_{o d d}$ and their product is $\chi_{0}$ since $\psi^{m}=\chi_{0}$. If $n$ is even, then $n=m$, and the stated result follows. If $n$ is odd, then $n=m+1$. We then take $\chi_{n}$ to be $\chi_{0}$.

To show the existence of $\varphi_{1}, \ldots, \varphi_{m}$, assume first that $4 \mid m$. Let $\Xi$ be a subgroup of $\widehat{\Omega}_{e v}$ of order 4. Any coset of $\Xi$ consists of four characters whose product is $\chi_{0}$. Thus, we can just choose the $\varphi_{i}$ 's so that $\left\{\varphi_{1}, \ldots, \varphi_{m}\right\}$ is a union of $m / 4$ distinct cosets of $\Xi$ in $\widehat{\Omega}_{e v}$. 
On the other hand, if $4 \nmid m$, then the inequalities show that $t \geq 5$. Thus, $\left[\widehat{\Omega}_{e v}: \Xi\right] \geq 4$. The bound on $n$ corresponds to the inequality $6 \leq m \leq 2^{t-1}-6$. First consider $m=6$. Suppose that the nontrivial elements of $\Xi$ are $\xi_{1}, \xi_{2}$, and $\xi_{3}$. Suppose that $\varphi$ and $\varphi^{\prime}$ belong to distinct, nontrivial cosets of $\Xi$ in $\widehat{\Omega}_{e v}$. Then

$$
A=\left\{\xi_{1}, \xi_{2}, \varphi \xi_{1}, \varphi \xi_{3}, \varphi^{\prime} \xi_{2}, \varphi^{\prime} \xi_{3}\right\}
$$

has cardinality $m$ and the product of the characters in $A$ is indeed $\chi_{0}$. Those characters belong to a union of three of the cosets of $\Xi$. If $m>6$, then one can form a set $A \cup B$, where $B$ is a union of $(m-6) / 4$ of the remaining cosets of $\Xi$ in $\widehat{\Omega}_{e v}$. The product of the elements in $A \cup B$ will again be $\chi_{0}$. This will settle all the $m$ 's in the indicated range.

Remark 6.2.3. If $K$ is a compositum of quadratic fields and $t \geq 3$, then one can simply take $\left\{\chi_{1}, \ldots, \chi_{n}\right\}$ to be either $\widehat{\Omega}_{o d d}$ or $\widehat{\Omega}_{o d d} \cup\left\{\chi_{0}\right\}$. The requirements in proposition 6.1 .1 are then satisfied for $n=2^{t-1}$ or $n=2^{t-1}+1$, respectively. Thus, if $K$ is $p$-rational, then one obtains homomorphisms $\rho_{0}$ and $\rho$ with the properties in proposition 6.1.1 for those values of $n$ too. For the special case $t=5$, we gave examples of suitable compositums of quadratic fields when $p=3$ and $p=5$ in section 4 . Thus, using those specific $K$ 's, we obtain representations $\rho: G_{\mathbf{Q}} \rightarrow G L_{n}\left(\mathbf{Z}_{p}\right)$ with an open image for both of those primes when $4 \leq n \leq 13$ and for $n=16$ and $n=17$ too. Using the example for $p=3$ with $t=6$, one obtains such $\rho$ 's for $4 \leq n \leq 29$ and for $n=32$ and 33. If one grants conjecture 4.2.1, then proposition 6.1.1 will give such representations for all pairs $(n, p)$, where $p \geq 3$ and $n \geq 4$.

For $n=2$, the requirements that $\chi_{1} \chi_{2}=\chi_{0}$ and $\chi_{1} \neq \chi_{2}$ imply that $\chi_{1}$ and $\chi_{2}$ have order at least 3. Similarly, if $n=3$, the requirements on $\chi_{1}, \chi_{2}$, and $\chi_{3}$ imply that one of those three characters is $\chi_{0}$ and the other two have order at least 3. Thus, compositums of quadratic fields will not work in these cases. However, if $p>3$, then one can apply proposition 6.1 .1 for $n=2$ or $n=3$ provided that one can find a cyclic extension $K$ of $\mathbf{Q}$ with the following two properties: (i) $K$ is complex and p-rational, (ii) the degree $[K: \mathbf{Q}]$ is at least 4 and divides $p-1$. It is reasonable to conjecture that such a field $K$ should exist for any prime $p$. The remaining cases are $(n, p)=(2,3)$ and $(n, p)=(3,3)$, both of which resist the approach of this paper. Of course, elliptic curves give many examples of 2-dimensional representations over $\mathbf{Z}_{3}$ with open image. We haven't found a way to construct 3-dimensional representations over $\mathbf{Z}_{3}$ with open image.

\section{Examples with irreducible residual representation.}

Suppose that $K$ is a finite Galois extension of $\mathbf{Q}$. We will assume in this section that $K$ is totally complex and that $\Omega=\operatorname{Gal}(K / \mathbf{Q})$ has order prime to $p$. We continue to assume that $p$ is an odd prime. As before, let $M$ denote the maximal pro- $p$ extension of $K$ which is unramified at all $\ell \notin \Sigma_{p}$. Let $\Gamma=\operatorname{Gal}(M / K)$. We will also assume that $K$ is $p$-rational in the main result and the 
illustrations in this section. Consequently, $\Gamma=\operatorname{Gal}(M / K)$ will be a free pro- $p$ group. We identify $\Omega$ with a subgroup of $\operatorname{Gal}(M / \mathbf{Q})$. Then $\Gamma$ becomes an $\Omega$-group and $\operatorname{Gal}(M / \mathbf{Q})$ is isomorphic to the corresponding semidirect product $\Gamma \rtimes \Omega$.

Suppose that $\mathcal{F}$ is a finite extension of $\mathbf{Q}_{p}, \mathfrak{m}$ is the maximal ideal in the ring of integers $\mathcal{O}$ of $\mathcal{F}$, and $\mathfrak{f}=\mathcal{O} / \mathfrak{m}$ is the corresponding residue field. Let $\mathcal{R}_{\mathcal{F}}(\Omega)$ and $\mathcal{R}_{\mathfrak{f}}(\Omega)$ denote the Grothendieck groups for representations of $\Omega$ over $\mathcal{F}$ and $\mathfrak{f}$, respectively. Then the natural map $\mathcal{R}_{\mathcal{F}}(\Omega) \rightarrow \mathcal{R}_{\mathfrak{f}}(\Omega)$ is an isomorphism. (See the references to [Ser] cited in remark 2.2.1.) The map is defined as follows. If $\omega$ is a representation of $\Omega$ over $\mathcal{F}$, then one can realize $\omega$ over $\mathcal{O}$. Let $\bar{\omega}$ denote the reduction of $\omega$ modulo $\mathfrak{m}$. The image of the isomorphism class of $\omega$ is defined to be the isomorphism class of $\bar{\omega}$ as an $\mathfrak{f}$-representation space for $\Omega$, which is well-defined because $|\Omega|$ is not divisible by $p$. If $\omega_{1}$ and $\omega_{2}$ are arbitrary $\mathcal{F}$-representations of $\Omega$, then $\omega_{1}$ is a direct summand in $\omega_{2}$ if and only if $\bar{\omega}_{1}$ is a direct summand in $\bar{\omega}_{2}$. All finite-dimensional representations of $\Omega$ over $\mathcal{F}$ and $\mathfrak{f}$ are completely reducible. In particular, note that if $\omega$ is an absolutely irreducible representation of $\Omega$ over $\mathbf{Q}_{p}$, then $\bar{\omega}$ is an absolutely irreducible representation of $\Omega$ over $\mathbf{F}_{p}$.

As before, let $\Omega_{\infty}$ be the decomposition subgroup for an infinite prime of $K$, a subgroup of $\Omega$ of order 2 , and let $\varepsilon_{1}$ be the nontrivial character of $\Omega_{\infty}$, regarded as a character with values in $\mathbf{Z}_{p}^{\times}$. Then $\operatorname{Ind}_{\Omega_{\infty}}^{\Omega}\left(\varepsilon_{1}\right)$ is a representation of $\Omega$ over $\mathbf{Q}_{p}$ of degree $\frac{1}{2}[K: \mathbf{Q}]$. The Frobenius Reciprocity Law implies that if $\omega$ is an absolutely irreducible representation of $\Omega$, then the multiplicity of $\omega$ in $\operatorname{Ind}_{\Omega_{\infty}}^{\Omega}\left(\varepsilon_{1}\right)$ (extending scalars if necessary) coincides with the multiplicity of $\varepsilon_{1}$ in $\left.\omega\right|_{\Omega_{\infty}}$. Thus, that multiplicity is positive unless $\Omega_{\infty} \subseteq \operatorname{ker}(\omega)$, i.e., unless $\omega$ factors through $\operatorname{Gal}\left(K^{\prime} / \mathbf{Q}\right)$, where $K^{\prime}$ is a totally real Galois extension of $\mathbf{Q}$ contained in $K$.

7.1. The basic proposition. The result below is a straightforward consequence of proposition 2.3.1. We will let $\omega_{0}$ denote the trivial representation of $\Omega$ in this section. Note that for any representation $\omega$ of $\Omega$ over a field $\mathcal{F}$, the representation $\omega \otimes_{\mathcal{F}} \check{\omega}$ contains $\omega_{0}$ with positive multiplicity. That multiplicity is 1 if and only if $\omega$ is absolutely irreducible. We will write the above tensor product more simply as $\omega \otimes \check{\omega}$.

Proposition 7.1.1. In addition to the above assumptions, suppose that $K$ is p-rational. Let $\omega$ be a representation of $\Omega$ over $\mathbf{Q}_{p}$ of degree $n$. Assume that $\omega \otimes \check{\omega}$ is a direct summand in $\operatorname{Ind}_{\Omega_{\infty}}^{\Omega}\left(\varepsilon_{1}\right) \oplus \omega_{0}$. Then there exists a representation $\rho: \operatorname{Gal}(M / \mathbf{Q}) \rightarrow G L_{n}\left(\mathbf{Z}_{p}\right)$ with open image such that $\bar{\rho}$ is isomorphic to $\bar{\omega}$.

The assumption about the tensor product implies that $\omega$ is absolutely irreducible. This is so because Frobenius reciprocity implies that $\omega_{0}$ is not a constituent in $\operatorname{Ind}_{\Omega_{\infty}}^{\Omega}\left(\varepsilon_{1}\right)$ and hence occurs with multiplicity 1 in $\omega \otimes \check{\omega}$. Therefore, since $\Omega$ has order prime to $p$, $\bar{\omega}$ will also be absolutely irreducible.

Proof. We can realize $\omega$ as a homomorphism from $\Omega$ to $G L_{n}\left(\mathbf{Z}_{p}\right)$. As described in section 5.6, the pro- $p$ group $C_{n}(p)$ then becomes an $\Omega$-group. It is clear that there is a homomorphism from 
the corresponding semidirect product $C_{n}(p) \rtimes \Omega$ to $G L_{n}\left(\mathbf{Z}_{p}\right)$ whose image contains $C_{n}(p)$ and is therefore an open subgroup of $G L_{n}\left(\mathbf{Z}_{p}\right)$. The assumptions together with proposition 3.2.1, remark 3.2.3, and the discussion in section 5.6 imply that $\widetilde{C_{n}(p)}$ is a direct summand in $\widetilde{\Gamma}$. Proposition 2.3.1 then implies that there is a surjective $\Omega$-homomorphism from $\Gamma$ to $C_{n}(p)$. Consequently, there is a surjective homomorphism from $\operatorname{Gal}\left(M / \mathbf{Q}\right.$ ) (which is isomorphic to $\Gamma \rtimes \Omega$ ) to $C_{n}(p) \rtimes \Omega$, and hence indeed to an open subgroup of $G L_{n}\left(\mathbf{Z}_{p}\right)$.

7.2. The deformation theory point of view. Let $G=\operatorname{Gal}(M / \mathbf{Q})$. Suppose that $\omega$ satisfies the hypotheses in proposition 7.1.1. Then the $\mathbf{F}_{p}$-representation $\bar{\omega}$ of $\Omega$ is absolutely irreducible. There exists a universal deformation $\operatorname{ring} R$ for the pair $G$ and $\bar{\omega}$. Thus, $R$ is a complete Noetherian local ring with residue field $\mathbf{F}_{p}$ and there exists a continuous representation $\rho_{\text {univ }}: G \rightarrow G L_{n}(R)$ whose residual representation is isomorphic to $\bar{\omega}$. A continuous ring homomorphism $\varphi: R \rightarrow \mathbf{Z}_{p}$ gives rise to a continuous representation $\rho_{\varphi}: G \rightarrow G L_{n}\left(\mathbf{Z}_{p}\right)$ with residual representation isomorphic to $\bar{\omega}$. Similarly, if we have such a homomorphism $\psi: R \rightarrow \mathbf{Z} / p^{2} \mathbf{Z}$, we obtain a representation $\rho_{\psi}: G \rightarrow G L_{n}\left(\mathbf{Z} / p^{2} \mathbf{Z}\right)$.

Suppose that the hypothesis concerning $\omega \otimes \check{\omega}$ in proposition 7.1.1 is satisfied. As in the proof of the proposition, it follows that there is a surjective $\Omega$-homomorphism from $\widetilde{\Gamma}$ to $\widetilde{C_{n}(p)}$. Now $\widetilde{C_{n}(p)}$ can be identified with a subgroup of $G L_{n}\left(\mathbf{Z} / p^{2} \mathbf{Z}\right)$, namely the kernel of the map $G L_{n}\left(\mathbf{Z} / p^{2} \mathbf{Z}\right) \rightarrow$ $G L_{n}(\mathbf{Z} / p \mathbf{Z})$. The homomorphism $\omega$ induces a homomorphism of $\Omega$ into $G L_{2}\left(\mathbf{Z} / p^{2} \mathbf{Z}\right)$. Consequently, there exists a homomorphism from $\widetilde{C_{n}(p)} \rtimes \Omega$ to $G L_{n}\left(\mathbf{Z} / p^{2} \mathbf{Z}\right)$ whose image contains $\widetilde{C_{n}(p)}$.

Recall that $L$ is the extension of $K$ contained in $M$ such that $\operatorname{Gal}(L / K) \cong \widetilde{\Gamma} \cdot \operatorname{Now} \operatorname{Gal}(L / \mathbf{Q}) \cong$ $\widetilde{\Gamma} \rtimes \Omega$ and hence there is a surjective homomorphism from $\operatorname{Gal}(L / \mathbf{Q})$ to $\widetilde{C_{n}(p)} \rtimes \Omega$. Thus, we obtain a homomorphism $\sigma$ from $\operatorname{Gal}(L / \mathbf{Q})$ to $G L_{n}\left(\mathbf{Z} / p^{2} \mathbf{Z}\right)$ whose image contains $\widetilde{C_{n}(p)}$. The corresponding residual representation is isomorphic to $\bar{\omega}$. We can regard $\sigma$ as a representation of $G$ over the ring $\mathbf{Z} / p^{2} \mathbf{Z}$. Therefore, there must be a continuous, surjective homomorphism $\psi: R \rightarrow \mathbf{Z} / p^{2} \mathbf{Z}$ such that $\rho_{\psi}=\sigma$. Furthermore, if there exists a continuous $\mathbf{Z}_{p}$-algebra homomorphism $\varphi: R \rightarrow \mathbf{Z}_{p}$ lifting $\psi$, then we obtain a representation $\rho_{\varphi}: G \rightarrow G L_{n}\left(\mathbf{Z}_{p}\right)$ whose residual representation is isomorphic to $\bar{\omega}$ and whose reduction modulo $p^{2}$ is $\sigma$. It follows from BBT that the image of $\rho_{\varphi}$ contains $C_{n}(p)$ and hence is an open subgroup of $G L_{n}\left(\mathbf{Z}_{p}\right)$.

The above remarks show that if one makes the assumptions that $\omega \otimes \check{\omega}$ is a direct summand in $\operatorname{Ind}_{\Omega_{\infty}}^{\Omega}\left(\varepsilon_{1}\right) \oplus \omega_{0}$ and that every continuous $\mathbf{Z}_{p}$-algebra homomorphism $\psi: R \rightarrow \mathbf{Z} / p^{2} \mathbf{Z}$ can be lifted to a continuous $\mathbf{Z}_{p}$-algebra homomorphism $\varphi: R \rightarrow \mathbf{Z}_{p}$, then one will obtain continuous representations $\rho: G \rightarrow G L_{n}\left(\mathbf{Z}_{p}\right)$ with open image and residual representation isomorphic to $\bar{\omega}$. It is not clear what assumptions about $R$ guarantee the existence of such liftings. If it happens that $R$ is isomorphic to a formal power series ring $\mathbf{Z}_{p}\left[\left[X_{1}, \ldots, X_{t}\right]\right]$ for some $t \geq 0$, then it is clear that the above lifting property holds. One sufficient condition for $R$ to have that structure is given in proposition 2 in [Maz]. It suffices to have $H^{2}(G, a d(\bar{\omega}))=0$. This is the so-called unobstructed 
case in deformation theory.

Recall that $K$ is $p$-rational if and only if $H^{2}(\Gamma, \mathbf{Z} / p \mathbf{Z})=0$. By Shapiro's lemma, another equivalent statement is that $H^{2}\left(G, \mathbf{F}_{p}[\Omega]\right)=0$, where $\mathbf{F}_{p}[\Omega]$ is the regular representation of $\Omega$, regarded as a $G$-module. This vanishing statement is in turn equivalent to the vanishing of $H^{2}(G, \alpha)$ for all $\alpha \in \operatorname{Irr}_{\mathbf{F}_{p}}(\Omega)$. In particular, if $K$ is $p$-rational, then it follows that $H^{2}(G, a d(\bar{\omega}))=0$ since $a d(\bar{\omega})$ is isomorphic to a direct sum of irreducible $\mathbf{F}_{p}$-representation spaces. Sometimes, it turns out that the vanishing of $H^{2}(G, a d(\bar{\omega}))$ and of $H^{2}\left(G, \mathbf{F}_{p}[\Omega]\right)$ are equivalent. As an example, suppose that $\Omega$ is the simple group of order 168 , whose representation theory will be discussed below, and that $\omega$ is either the irreducible representation of degree 7 or of degree 8 . Then every irreducible representation of $\Omega$ is a constituent in $\omega \otimes \check{\omega}$, as we point out below. It then follows that every $\alpha$ in $\operatorname{Irr}_{\mathbf{F}_{p}}(\Omega)$ occurs as a constituent in $a d(\bar{\omega})$. The vanishing of $H^{2}(G, a d(\bar{\omega}))$ and of $H^{2}\left(G, \mathbf{F}_{p}[\Omega]\right)$ are indeed equivalent. Consequently, the field $K$ is $p$-rational if and only if $H^{2}(G, a d(\bar{\omega}))=0$.

As a simpler example, suppose that $\Omega=\operatorname{Gal}(K / \mathbf{Q})$ is isomorphic to $S_{3}$ and that $\omega$ is the 2 -dimensional irreducible representation of $\Omega$. Assume that $p \geq 5$. Then all three elements of $\operatorname{Irr}_{\mathbf{F}_{p}}(\Omega)$ are constituents in $\operatorname{ad}(\bar{\omega})$. Hence $K$ is $p$-rational if and only if $H^{2}(G, a d(\bar{\omega}))$ vanishes. In this special case, that vanishing means that $R$ is isomorphic to a formal power series ring over $\mathbf{Z}_{p}$ in either one or three variables, depending on whether $K$ is totally real or totally complex. In [Maz], Mazur considers certain examples of totally complex $S_{3}$-extensions $K$ of $\mathbf{Q}$ which he calls "special". These extensions correspond to primes $p$ of the form $p=27+4 a^{3}$. For each such prime $p$, Let $K$ be the splitting field over $\mathbf{Q}$ for the polynomial $x^{3}+a x+1$. There are eighteen such primes $p<10^{6}$ and the field $K$ turns out to be $p$-rational for each of them.

7.3. An illustration. We discuss the example mentioned in the introduction. Thus, we will suppose that $\Omega=\operatorname{Gal}(K / \mathbf{Q})$ is isomorphic to the symmetric group $S_{n+1}$ for some $n$. It is known that such totally complex Galois extensions $K / \mathbf{Q}$ exist for all $n \geq 0$. We fix an isomorphism. Suppose that $n \geq 2$. Then $S_{n+1}$ has an absolutely irreducible representation of degree $n$, a direct summand in the obvious permutation representation of degree $n+1$. We will let $\omega_{\boldsymbol{n}}$ be the corresponding representation of $\Omega$. All the representations of the symmetric groups are self-dual. In particular, $\check{\omega}_{n} \cong \omega_{\boldsymbol{n}}$.

Proposition 7.3.1. If $n=2$ or $n \geq 4$, then the representation $\omega_{\boldsymbol{n}} \otimes \omega_{\boldsymbol{n}}$ is a direct summand in $\operatorname{Ind}_{\Omega_{\infty}}^{\Omega}\left(\varepsilon_{1}\right) \oplus \omega_{0}$. If $n=3$, this statement is true if the unique quadratic subfield of $K$ is imaginary.

The proof depends on the lemma below. We will use various results about the representation theory of $S_{n+1}$ which can be found in [JaKe]. We think of $S_{n+1}$ as the group of permutations of a set $X_{n+1}=\left\{x_{1}, \ldots, x_{n+1}\right\}$. First of all, the absolutely irreducible representations of symmetric groups are always realizable over $\mathbf{Q}$. This is theorem 2.1.3 in [JaKe]. In particular, they are all self-dual. That latter fact is clear because if $g \in S_{n+1}$, then $g$ and $g^{-1}$ have the same cycle decomposition and hence are conjugate. This implies that the characters of all representations of $S_{n+1}$ are real-valued. 
In fact, $g$ is conjugate to any generator of the cyclic group $\langle g\rangle$ generated by $g$ and this implies that all these characters have values in $\mathbf{Q}$. The fact that the corresponding representations can be defined over $\mathbf{Q}$ is somewhat harder to show.

The isomorphism classes of irreducible representations of $S_{n+1}$ correspond to partitions of $n+1$ as sums of positive integers. For any such partition $n+1=a_{1}+\ldots+a_{t}$, where $t \geq 0$, we assume that the terms are arranged so that $a_{i} \geq a_{i+1}$ for $1 \leq i<t$. The corresponding irreducible representation will be denoted by $\left[a_{1}, \ldots, a_{t}\right]$. This notation (which is taken from [JaKe]) indicates in part that this irreducible representation is a constituent in $\operatorname{Ind}_{H}^{S_{n+1}}\left(\mathbf{1}_{H}\right)$, where $H$ is a subgroup of $S_{n+1}$ isomorphic to the direct product $S_{a_{1}} \times \ldots \times S_{a_{t}}$ and is defined by expressing the set $X_{n+1}$ as a disjoint union of $t$ subsets with cardinalities $a_{1}, \ldots, a_{t}$. Here $\mathbf{1}_{H}$ is the trivial representation of $H$. To uniquely determine $\left[a_{1}, \ldots, a_{t}\right]$, one requires also that the twist $\left[a_{1}, \ldots, a_{t}\right] \otimes s g n$ is a constituent in $\operatorname{Ind}_{H^{\prime}}^{S_{n+1}}\left(\mathbf{1}_{H^{\prime}}\right)$, where $\operatorname{sgn}$ is the nontrivial character of $S_{n+1} / A_{n+1}$ and $H^{\prime}$ is a subgroup of $S_{n+1}$ defined just as above, but corresponding to another partition $n+1=a_{1}^{\prime}+\ldots+a_{t^{\prime}}^{\prime}$ of $n+1$ which is specified in the following way. The original partition defines a matrix $A$ with $t$ rows and $a_{1}$ columns, where the $i$-th row has the first $a_{i}$ entries equal to 1 and the remaining entries (if any) equal to 0 . Thus, the total number of 1 's in the matrix $A$ is $n+1$. The second partition is defined to be the one whose corresponding matrix $A^{\prime}$ is the transpose of $A$. Thus, $a_{1}^{\prime}=t$ and $t^{\prime}=a_{1}$.

The trivial representation of $S_{n+1}$ corresponds to the partition with $t=1$, and is denoted by $[n+1]$. The natural permutation representation of $S_{n+1}$ is $\operatorname{Ind}_{S_{n}}^{S_{n+1}}\left(\mathbf{1}_{S_{n}}\right)$, where $S_{n}$ is identified with the subgroup of $S_{n+1}$ fixing $x_{n+1}$. That induced representation has an irreducible constituent of degree $n$ which corresponds to the partition $n+1=a_{1}+a_{2}$, with $a_{1}=n, a_{2}=1$, and is the irreducible representation $[n, 1]$. Note that $\mathbf{1}_{S_{n}}$ is $[n]$. We prove the following lemma:

Lemma 7.3.2. If $n \geq 3$, then we have an isomorphism

$$
[n, 1] \otimes[n, 1] \cong[n+1] \oplus[n, 1] \oplus[n-1,2] \oplus[n-1,1,1]
$$

as representations of $S_{n+1}$. In particular, each irreducible constituent in $[n, 1] \otimes[n, 1]$ has multiplicity 1. The only irreducible constituent of degree 1 is $[n+1]$.

Although we won't need it, the degrees of the four constituents in $[n, 1] \otimes[n, 1]$ turn out to be $1, n, \frac{1}{2}(n+1)(n-2)$, and $\frac{1}{2} n(n-1)$, respectively. One sees this by using theorem 2.3.21 in [JaKe].

Proof of the lemma. Identifying $S_{n}$ with a subgroup of $S_{n+1}$ as above, we have

$$
([n, 1] \otimes[n, 1]) \oplus[n, 1] \cong([n, 1] \oplus[n+1]) \otimes[n, 1] \cong \operatorname{Ind}_{S_{n}}^{S_{n+1}}([n]) \otimes[n, 1] .
$$

One sees easily from the definitions that the restriction of the natural permutation representation of $S_{n+1}$ to the subgroup $S_{n}$ is isomorphic to the direct $\operatorname{sum}[n-1,1] \oplus[n] \oplus[n]$. It follows that the 
restriction of $[n, 1]$ to $S_{n}$ is isomorphic to the direct sum $[n-1,1] \oplus[n]$. Using a standard property of induction, we have

$$
\operatorname{Ind}_{S_{n}}^{S_{n+1}}([n]) \otimes[n, 1] \cong \operatorname{Ind}_{S_{n}}^{S_{n+1}}([n-1,1] \oplus[n]) \cong \operatorname{Ind}_{S_{n}}^{S_{n+1}}([n-1,1]) \oplus \operatorname{Ind}_{S_{n}}^{S_{n+1}}([n])
$$

The Branching Theorem, which is theorem 2.4.2 in [JaKe], gives the decomposition of $\operatorname{Ind}_{S_{n}}^{S_{n+1}}(\chi)$ if $\chi$ is an irreducible representation of $S_{n}$. Suppose that $\chi$ corresponds to the partition $n=$ $a_{1}+\ldots+a_{t}$, as above, where $t \geq 1$. We can imagine this partition of $n$ as a partition with $t+1$ terms by setting $a_{t+1}=0$. We can form various partitions of $n+1$ by replacing exactly one of the summands $a_{i}$ by $a_{i}+1$. We consider only the resulting partitions where the summands are in nondecreasing order. Thus, if some of the $a_{i}$ 's are repeated, we need only augment the first such summand by 1 . Then $\operatorname{Ind}_{S_{n}}^{S_{n+1}}(\chi)$ is isomorphic to the direct sum of the irreducible representations which corresponds to the various partitions of $n+1$ obtained as just described. In particular, we have

$\operatorname{Ind}_{S_{n}}^{S_{n+1}}([n]) \cong[n+1] \oplus[n, 1], \quad \operatorname{Ind}_{S_{n}}^{S_{n+1}}([n-1,1]) \cong[n, 1] \oplus[n-1,2] \oplus[n-1,1,1]$.

The first isomorphism amounts to the definition that we gave before for the irreducible representation $[n, 1]$ of $S_{n+1}$ of degree $n$. The isomorphism in the lemma follows directly from the above isomorphisms.

Proof of the proposition. If $n=2$, then $\Omega \cong S_{3}$ has three nonisomorphic irreducible representations and one checks easily that $\omega_{\mathbf{2}} \otimes \omega_{\mathbf{2}}$ is isomorphic to their direct sum, each with multiplicity 1 . However, $\operatorname{Ind}_{\Omega_{\infty}}^{\Omega}\left(\varepsilon_{1}\right) \oplus \omega_{0}$ is also isomorphic to that direct sum.

If $n=3$, then $\Omega \cong S_{4}$ and the stated assumption about $K$ implies that a generator of $\Omega_{\infty}$ corresponds to a transposition in the isomorphism. For any $n \geq 0$, the transpositions in $S_{n+1}$ are all conjugate and generate $S_{n+1}$. If $\omega$ is any irreducible representation of $\Omega$, and $\omega \neq \omega_{0}$, then it follows that $\Omega_{\infty} \not \subset \operatorname{ker}(\omega)$ and hence that $\left.\omega\right|_{\Omega_{\infty}}$ contains $\varepsilon_{1}$ as a constituent. Thus, every irreducible representation of $\Omega$ is a constituent in $\operatorname{Ind}_{\Omega_{\infty}}^{\Omega}\left(\varepsilon_{1}\right) \oplus \omega_{0}$. On the other hand, lemma 7.2.2 implies that the irreducible constituents in $\omega_{\mathbf{3}} \otimes \omega_{\mathbf{3}}$ have multiplicity 1. It follows that $\omega_{\mathbf{3}} \otimes \omega_{\mathbf{3}}$ is indeed a direct summand in $\operatorname{Ind}_{\Omega_{\infty}}^{\Omega}\left(\varepsilon_{1}\right) \oplus \omega_{0}$.

If $n \geq 4$, then $\Omega$ has just one proper normal subgroup. We denote that subgroup by $\Theta$. We have $[\Omega: \Theta]=2$. Of course, $\Theta$ corresponds to the alternating group $A_{n+1}$ under the isomorphism $\Omega \cong S_{n+1}$. There is a nontrivial representation $\omega_{1}$ of $\Omega$ whose kernel is $\Theta$. Apart from $\omega_{0}$ and $\omega_{\mathbf{1}}$, it follows that the remaining irreducible representations of $\Omega$ are all faithful. Hence, if $\omega$ is any such representation, $\varepsilon_{1}$ must occur as a constituent in $\left.\omega\right|_{\Omega_{\infty}}$. Frobenius reciprocity therefore implies that $\omega$ occurs as a constituent in $\operatorname{Ind}_{\Omega_{\infty}}^{\Omega}\left(\varepsilon_{1}\right)$. Thus, the proposition will follow if we show that each of the irreducible constituents in $\omega \otimes \omega$ has multiplicity 1 in that representation space and that $\omega_{1}$ does not occur as a constituent. The latter fact is needed because it is possible to have $\Omega_{\infty} \subset \Theta$ and $\omega_{1}$ will fail to be a constituent in $\operatorname{Ind}_{\Omega_{\infty}}^{\Omega}\left(\varepsilon_{1}\right)$ in that case. 
Lemma 7.3.2 shows that $\omega_{\boldsymbol{n}} \otimes \omega_{\boldsymbol{n}}$ has four irreducible constituents and that they are indeed nonisomorphic. The representation $\omega_{1}$ of $\Omega$ corresponds to the representation of $S_{n+1}$ with kernel $A_{n+1}$. The corresponding partition of $n+1$ has $t=n+1$ and all summands equal to 1 . This representation is $[1, \ldots, 1]$ and is not isomorphic to any of the four constituents in $[n, 1] \otimes[n, 1]$. Thus, the properties of $\omega_{\boldsymbol{n}} \otimes \omega_{\boldsymbol{n}}$ that we need are indeed true.

Remark 7.3.3. If $n=3$, then $\Omega$ contains a unique normal subgroup of order 4, the Klein 4-group, which we denote by $\Upsilon$. The quotient $\Omega / \Upsilon$ is isomorphic to $S_{3}$. If the unique quadratic subfield of $K$ is real, then $\Omega_{\infty} \subset \Upsilon$. There are five irreducible representations of $\Omega$. Three of them factor through $\Omega / \Upsilon$ and have degrees 1,1 , and 2 . The other two have degree 3 and are faithful. Let $\omega_{\mathbf{2}}$ denote the unique irreducible 2-dimensional representation of $\Omega$. In fact, $\omega_{\mathbf{2}}$ corresponds to the representation $[2,2]$ of $S_{4}$. We have $\operatorname{ker}\left(\omega_{2}\right)=\Upsilon$. Frobenius reciprocity implies that $\omega_{2}$ is not a constituent in $\operatorname{Ind}_{\Omega_{\infty}}^{\Omega}\left(\varepsilon_{1}\right)$. However, $\omega_{\mathbf{2}}$ is a constituent in $\omega_{\mathbf{3}} \otimes \omega_{\mathbf{3}}$. Therefore the assumption in proposition 7.3 .1 is not satisfied in this case.

7.4. Additional illustrations. Propositions 7.1.1 and 7.3.1 provide a possible source of examples of continuous $n$-dimensional representations of $G_{\mathbf{Q}}$ over $\mathbf{Q}_{p}$ with open image and absolutely irreducible residual representation. However, if $n \geq 3$ and $p$ is any prime, then it would seem rather difficult to determine whether an extension $K / \mathbf{Q}$ with $\operatorname{Gal}(K / \mathbf{Q}) \cong S_{n+1}$ is actually $p$-rational. We have not made any attempt to do such a computation. If one does manage to find such a field, then one can apply proposition 7.1.1 to other irreducible representations of $\mathrm{Gal}(\mathrm{K} / \mathbf{Q})$ of various dimensions. We give several illustrations.

7.4.1. $\Omega \cong S_{5}$. Suppose that $K$ is totally complex and is $p$-rational for some prime $p \geq 7$. The elements of order 2 in $S_{5}$ form two conjugacy classes, one consisting of the transpositions, the other consisting of products of two disjoint transpositions. Thus, there are two possible conjugacy classes for $\Omega_{\infty}$. The unique quadratic subfield of $K$ is imaginary when $\Omega_{\infty}$ corresponds to a subgroup of $S_{5}$ generated by a transposition. For the other possibility for $\Omega_{\infty}$, that quadratic subfield will be real. Now $S_{5}$ has seven irreducible representations. In addition to the two one-dimensional representations factoring through $S_{5} / A_{5}$, there are two of degree 4 , two of degree 5 , and one of degree 6 , up to isomorphism. We denote the corresponding irreducible representations of $\Omega$ by $\omega_{0}, \omega_{\mathbf{1}}, \omega_{\mathbf{4}, 1}, \omega_{\mathbf{4}, 2}, \omega_{\mathbf{5}, 1}, \omega_{\mathbf{5}, 2}$, and $\omega_{\mathbf{6}}$. The degrees of the nontrivial representations listed here are indicated by boldface subscripts. To be more precise, $\omega_{\mathbf{4}, 1}$ corresponds to $[4,1], \omega_{\mathbf{4}, 2}=\omega_{\mathbf{4}, 1} \otimes \omega_{\mathbf{1}}$ corresponds to $[2,1,1,1], \omega_{\mathbf{5}, 1}$ corresponds to $[3,2]$, and $\omega_{\mathbf{5}, 2}=\omega_{\mathbf{5}, 1} \otimes \omega_{\mathbf{1}}$ corresponds to $[2,2,1]$. The trivial representation is $\omega_{0}$.

If the unique quadratic subfield of $K$ is imaginary, then one finds that

$$
\operatorname{Ind}_{\Omega_{\infty}}^{\Omega}\left(\varepsilon_{1}\right) \cong \omega_{\mathbf{1}} \oplus \omega_{\mathbf{4}, 1} \oplus \omega_{\mathbf{4}, 2}^{3} \oplus \omega_{\mathbf{5}, 1}^{2} \oplus \omega_{\mathbf{5}, 2}^{3} \oplus \omega_{\mathbf{6}}^{3} .
$$

On the other hand, if $\omega$ is any one of the irreducible representations of $\Omega$, the decomposition of the tensor product $\omega \otimes \omega$ can be found in the table in [JaKe] starting on page 451 . One finds 
that all of the irreducible constituents in $\omega \otimes \omega$ have multiplicity 1 , except for the case $\omega=\omega_{6}$. If $\omega=\omega_{\mathbf{6}}$, then all of the irreducible constituents in $\omega \otimes \omega$ have multiplicity 1 , except for $\omega_{\mathbf{5}, 1}$ and $\omega_{\mathbf{5}, 2}$, both of which have multiplicity 2. Thus, for any irreducible $\omega$, the hypotheses in proposition 7.1.1 are satisfied and consequently, under the assumption that $p \geq 7$ and that $K$ is $p$-rational, there would then exist continuous $n$-dimensional representations of $G_{\mathbf{Q}}$ with open image and absolutely irreducible residual representation (isomorphic to $\bar{\omega}$ ) for $n=4,5$, and 6 .

If the unique quadratic subfield of $K$ is real, then it turns out that

$$
\operatorname{Ind}_{\Omega_{\infty}}^{\Omega}\left(\varepsilon_{1}\right) \cong \omega_{\mathbf{4 , 1}}^{2} \oplus \omega_{\mathbf{4}, 2}^{2} \oplus \omega_{\mathbf{5}, 1}^{2} \oplus \omega_{\mathbf{5}, 2}^{2} \oplus \omega_{\mathbf{6}}^{4}
$$

Note that $\omega_{1}$ is not a constituent in this induced representation. Now it turns out that $\omega_{1}$ is a constituent (with multiplicity 1) in $\omega_{\mathbf{6}} \otimes \omega_{\mathbf{6}}$, but is not a constituent in $\omega \otimes \omega$ for the other irreducible representations of $\Omega$. Thus, the hypotheses in proposition 7.1 .1 are satisfied for all the irreducible representations $\omega$ of $\Omega$, except for $\omega_{\mathbf{6}}$. For any such $\omega$, one would obtain a continuous representation $\rho$ of $G_{\mathbf{Q}}$ with open image such that $\bar{\rho} \cong \bar{\omega}$, again under the assumption that $K$ is $p$-rational. The dimension would be be 4 or 5 .

7.4.2. $\Omega \cong A_{5}$. Thus, $\Omega$ is the simple group of order 60 . We still assume that $p \geq 7$ and that $K$ is totally complex and $p$-rational. In this case, there is only one possible conjugacy class for $\Omega_{\infty}$. There are five irreducible representations of $\Omega$. In addition to $\omega_{0}$, two have degree 3 , one has degree 4 , and one has degree 5 . The ones of degree 4 and 5 are the restrictions of representations of $S_{5}$ and hence are realizable over $\mathbf{Q}$. The two of degree 3 are the irreducible constituents of $\left.\omega_{6}\right|_{A_{5}}$ and are realizable over the field $\mathbf{Q}(\sqrt{5})$. All of these representations are self-dual. One finds that $\operatorname{Ind}_{\Omega_{\infty}}^{\Omega}\left(\varepsilon_{1}\right)$ is isomorphic to the direct sum of the four nontrivial irreducible representations, all with multiplicity 2. Furthermore, if $\omega$ is any one of the irreducible representations of $\Omega$, one finds that the hypothesis about $\omega \otimes \omega$ in proposition 7.1.1 is again satisfied. Thus, if one can find such a field $K$, one would then obtain continuous $n$-dimensional representations of $G_{\mathbf{Q}}$ over $\mathbf{Q}_{p}$ with open image and irreducible residual representation for $n=4$ or 5 . The same thing is true for $n=3$ if one assumes that $p$ splits in the quadratic field $\mathbf{Q}(\sqrt{5})$.

7.4.3. $\Omega \cong S_{6}$. If $p \geq 7, K$ is totally complex and $p$-rational, and $\Omega \cong S_{6}$, then there are three possible conjugacy classes for $\Omega_{\infty}$. There are eleven irreducible representations of $S_{6}$. Their degrees are 1, 5, 9, 10, and 16. Up to isomorphism, there are four irreducible representations of degree 5 , two for each of the degrees 1, 9, and 10, and just one of degree 16. For all of these irreducible representations $\omega$ of $\Omega$, except for the one of degree 16, and for all three possibilities for the isomorphism class of $\operatorname{Ind}_{\Omega_{\infty}}^{\Omega}\left(\varepsilon_{1}\right)$, one finds that the hypothesis concerning $\omega \otimes \omega$ in proposition 7.1.1 is satisfied. To verify this, one can use the above cited tables in [JaKe] as before to determine the decomposition of the representation space $\omega \otimes \omega$. The character tables on page 350 in [JaKe] together with Frobenius reciprocity determine the decomposition of $\operatorname{Ind}_{\Omega_{\infty}}\left(\varepsilon_{1}\right)$. As a consequence, if one can find such a field $K$, one would then obtain continuous $n$-dimensional representations of $G_{\mathbf{Q}}$ with open image and irreducible residual representation for $n=5,9$, and 10 . 
7.4.4. $\Omega \cong P S L_{2}\left(\mathbf{F}_{7}\right)$. In this final illustration, $\Omega$ is the simple group of order 168 . At the end of [Ham], Hamblem discusses one such example due to W. Trinks, where $K$ is the splitting field for $x^{7}-7 x+3$ over $\mathbf{Q}$. There are two absolutely irreducible representations of $\Omega$ of degree 3 . Let $\omega$ be either one of them. Hamblen's main result in [Ham] can be applied to this example when $p \equiv 8(\bmod 21)$ to construct a 3 -dimensional representation $\rho$ of $G_{\mathbf{Q}}$ over $\mathbf{Q}_{p}$ with open image such that $\bar{\rho} \cong \bar{\omega}$. His representation $\rho$ is unramified outside a finite set containing $\Sigma_{p} \cup\{3,7, \infty\}$. Interestingly, his construction gives a representation $\rho$ such that $\rho\left(D_{p}\right)$ is upper triangular, where $D_{p}$ is the decomposition subgroup of $G_{\mathbf{Q}}$ for some prime above $p$.

In contrast, proposition 7.1.1 can be applied only if one can verify that $K$ is $p$-rational, where $p \notin\{2,3,7\}$. As with the other examples, we haven't attempted to do this, but we would expect that any fixed number field $K$ will turn out to be $p$-rational for almost all primes $p$. That is, the set of exceptions should have Dirichlet density 0. Furthermore, there should be infinitely many distinct Galois extensions $K$ of $\mathbf{Q}$ with Galois group isomorphic to $P S L_{2}\left(\mathbf{F}_{7}\right)$. Indeed, in [LaM], one finds an infinitely family of polynomials over $\mathbf{Q}$ of degree 7 with that Galois group. Although this is not stated, presumably the corresponding extensions will form an infinite family too. Thus, it seems exceedingly likely that, for every odd prime $p$, such a $K$ will exist which is $p$-rational.

The character table for $P S L_{2}\left(\mathbf{F}_{7}\right)$ can be found on page 318 in [JaLi]. The following remarks are derived from that table. In addition to the trivial representation $\omega_{0}$, there are five isomorphism classes of absolutely irreducible representations of $\Omega$. We denote them by $\omega_{\mathbf{3}, 1}, \omega_{\mathbf{3}, 2}, \omega_{\mathbf{6}}, \omega_{\mathbf{7}}$, and $\omega_{\mathbf{8}}$. The two representations of degree 3 are contragredients of each other. The others are self-dual. Furthermore, there is only one conjugacy class of elements of order 2 . Hence $\Omega_{\infty}$ is unique up to conjugacy and one finds that

$$
\operatorname{Ind}_{\Omega_{\infty}}^{\Omega}\left(\varepsilon_{1}\right) \cong \omega_{\mathbf{3}, 1}^{2} \oplus \omega_{\mathbf{3}, 2}^{2} \oplus \omega_{\mathbf{6}}^{2} \oplus \omega_{\mathbf{7}}^{4} \oplus \omega_{\mathbf{8}}^{4} .
$$

As for the tensor product occurring in proposition 7.1.1, the decomposition is as follows:

$$
\begin{gathered}
\omega_{\mathbf{3}, 1} \otimes \omega_{\mathbf{3}, 2} \cong \omega_{0} \oplus \omega_{\mathbf{8}}, \quad \omega_{\mathbf{7}} \otimes \omega_{\mathbf{7}} \cong \omega_{0} \oplus \omega_{\mathbf{3}, 1} \oplus \omega_{\mathbf{3}, 2} \oplus \omega_{\mathbf{6}}^{2} \oplus \omega_{\mathbf{7}}^{2} \oplus \omega_{\mathbf{8}}^{2}, \\
\omega_{\mathbf{6}} \otimes \omega_{\mathbf{6}} \cong \omega_{0} \oplus \omega_{\mathbf{6}}^{2} \oplus \omega_{\mathbf{8}}^{2}, \quad \omega_{\mathbf{8}} \otimes \omega_{\mathbf{8}} \cong \omega_{0} \oplus \omega_{\mathbf{3}, 1} \oplus \omega_{\mathbf{3}, 2} \oplus \omega_{\mathbf{6}}^{2} \oplus \omega_{\mathbf{7}}^{3} \oplus \omega_{\mathbf{8}}^{3} .
\end{gathered}
$$

Each of these tensor products is a direct summand in $\operatorname{Ind}_{\Omega_{\infty}}^{\Omega}\left(\varepsilon_{1}\right) \oplus \omega_{0}$.

Except for the irreducible representations of degree 3, all of the others have rational-valued characters. Furthermore, a theorem of Janusz states that if $\mathbf{F}$ is any finite field, then the Schur index over $\mathbf{Q}$ for any irreducible representation of $P S L_{2}(\mathbf{F})$ is 1 . In particular, the irreducible representations of degree 6,7 , and 8 for $\Omega$ can be realized over $\mathbf{Q}$ and therefore over $\mathbf{Q}_{p}$ for any prime $p$. One can easily verify this assertion about the Schur indices for $\mathbf{F}=\mathbf{F}_{7}$ by examining the multiplicity of any irreducible representation $\omega$ of $\Omega$ in $\operatorname{Ind}_{\Theta}^{\Omega}(\theta)$, where $\Theta$ is a Sylow 2-subgroup of $\Omega$ and $\theta$ is one of the three characters of $\Theta$ of order 2. Those induced representations are defined over $\mathbf{Q}$. For each $\omega$, and at least one choice of $\theta$, the multiplicity turns out to be 1 . One can check this by using the character table for $\Omega$ and Frobenius reciprocity. 
Therefore, assuming that $K$ is $p$-rational and that $p \geq 11$ or $p=5$, one would obtain continuous representations $\rho$ of $G_{\mathbf{Q}}$ of degrees 6 , 7, or 8 such that the image of $\rho$ is open and the residual representation $\bar{\rho}$ is an absolutely irreducible representation factoring through $\Omega$. One also obtains such representations of degree 3 if one makes the additional assumption that $p$ splits in the quadratic field $\mathbf{Q}(\sqrt{-7})$.

\section{References}

[Bos] N. Boston, Explicit deformations of Galois representations, Invent. Math. 103 (1991), 181196.

[DSMS] J. D. Dixon, M. P. F. du Sautoy, A. Mann, D. Segal, Analytic pro- $p$ groups, London Math. Soc. Lecture Note Series 157, Cambridge University Press (1991).

[Fuj] S. Fujii, On the maximal pro-p extension unramified outside $p$ of an imaginary quadratic field, Osaka J. Math. 45 (2008), 41-60.

[Gor] D. Gorenstein, Finite groups, Chelsea Publ. Co. (1980).

[Gr73] R. Greenberg, On a certain $\ell$-adic representation, Invent. Math. 21 (1973), 117-124.

[Gr1] R. Greenberg, Iwasawa Theory, Projective Modules, and Modular Representations, to appear in Memoirs of the Amer. Math. Soc.

[Gr2] R. Greenberg, Galois properties of elliptic curves with an isogeny, preprint.

[Ham] S. Hamblen, Lifting n-dimensional Galois representations, Can. Jour. of Math. 602008 , 1028-1049.

[HeRi] , W. N. Herfort, L. Ribes, On automorphisms of free pro-p-groups I, Proc. Amer. Math. Soc. 108 (1990), 287-295.

[Hub] , D. Hubbard, The nonexistence of certain free pro-p extensions and capitulation in a family of dihedral extensions of $\mathbf{Q}$, University of Washington Ph. D. thesis, 1996.

[JaKe] G. James, A. Kerber, The representation theory of the symmetric group, Encyclopedia of Mathematics and its Applications 16 (1981), Addison-Wesley.

[JaLi] G. James, M. Liebeck, Representations and characters of groups, 2001, Cambridge University Press.

[JaNg] J. F. Jaulent, T. Nguyen Quang Do, Corps p-rationnels, corps p-réguliers, et ramification restreinte, Séminaire de Théorie des Nombres de Bordeaux, (1987-88), Exposé 10, 10-01 $10-26$. 
[LaM] S. E. LaMacchia, Polynomials with Galois group PSL(2,7), Comm. in Algebra 8 (1980), $983-992$.

[Maz] B. Mazur, Deforming Galois representations, in Galois Groups over Q, Math. Sci. Res. Inst. Publ. 16, Springer, New York, 1989, 385-437.

[Min] J. Minardi, Iwasawa modules for $\mathbf{Z}_{p}^{d}$-extensions of algebraic number fields, University of Washington Ph. D. thesis, 1986.

[Mov] A. Movahhedi, Sur les p-extensions des corps p-rationnels, Math. Nach. 149 (1990), 163-176.

[MoNg] A. Movahhedi, T. Nguyen Quang Do, Sur l'arithmétique des corps de nombres prationnels, Prog. Math. 81, Birkhauser (1990), 155-200.

[Ngu] T. Nguyen Quang Do, Sur la $\mathbf{Z}_{p}$-torsion de certains modules Galoisiens, Ann. Inst. Fourier 36 (1986), 27-46.

[NSW] J. Neukirch, A. Schmidt, K. Wingberg, Cohomology of Number Fields, Grundlehren der Math. Wissenschaften 323 (2000), Springer.

[Ser] J. P. Serre, Linear representations of finite groups, Graduate Texts in Math. (1977), Springer.

[Sha] I. R. Shafarevich, Extensions with given points of ramification, Amer. Math. Soc. Translations 59 (1966), 128-149.

[Upt] M. G. Upton, Galois representations attached to Picard curves, Jour. Algebra 322 (2009), 1038-1059.

[Was] L. C. Washington, Introduction to Cyclotomic Fields, 2nd ed. Graduate Texts in Math. Springer (1997).

[Yam] A note on free pro-p-extensions of algebraic number fields, Jour. Théorie des Nombres de Bordeaux 5 (1993), 165-178. 